\title{
Beam Tube Vacuum in Future Superconducting Proton Colliders*
}

\author{
W. Turner \\ Superconducting Super Collider Laboratory ${ }^{\dagger}$ \\ 2275 North Hwy. 77 \\ Waxahachie, TX 75165
}

October 1994

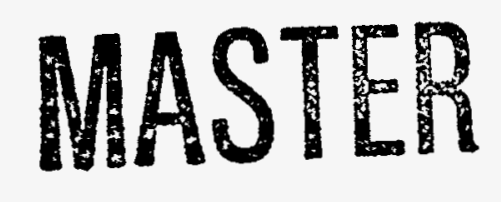

- To be published in Accelerator Physics at the Superconducting Super Collider.

t Operated by the Universities Research Association, Inc., for the U.S. Department of Energy under Contract No. DE-AC35-89ER40486.

DISTRIBUTION DE THIS DOCUMENT LS UNLIMITEA, 


\section{DISCLAIMER}

This report was prepared as an account of work sponsored by an agency of the United States Government. Neither the United States Government nor any agency thereof, nor any of their employees, make any warranty, express or implied, or assumes any legal liability or responsibility for the accuracy, completeness, or usefulness of any information, apparatus, product, or process disclosed, or represents that its use would not infringe privately owned rights. Reference herein to any specific commercial product, process, or service by trade name, trademark, manufacturer, or otherwise does not necessarily constitute or imply its endorsement, recommendation, or favoring by the United States Government or any agency thereof. The views and opinions of authors expressed herein do not necessarily state or reflect those of the United States Government or any agency thereof. 


\section{DISCLAIMER}

Portions of this document may be illegible in electronic image products. Images are produced from the best available original document. 
BEAM TUBE VACUUM IN FUTURE SUPERCONDUCTING PROTON COLLIDERS

\author{
WILLIAM C. TURNER
}

Superconducting Super Collider Laboratory, ${ }^{*}$ Waxahachie, Texas 75165

\begin{abstract}
The beam tube vacuum requirements in future superconducting proton colliders that have been proposed or discussed in the literature-SSC, LHC, and ELN-are reviewed. The main beam tube vacuum problem encountered in these machines is how to deal with the magnitude of gas desorption and power deposition by synchrotron radiation while satisfying resistivity, impedance, and space constraints in the cryogenic environment of superconducting magnets. A beam tube vacuum model is developed that treats photodesorption of tightly bound $\mathrm{H}, \mathrm{C}$, and $\mathrm{O}$, photodesorption of physisorbed molecules, and the isotherm vapor pressure of $\mathrm{H}_{2}$. Experimental data on cold tube photodesorption experiments are reviewed and applied to model calculations of beam tube vacuum performance for simple cold beam tube and liner configurations. Particular emphasis is placed on the modeling and interpretation of beam tube photodesorption experiments at electron synchrotron light sources. The paper also includes discussion of the constraints imposed by beam image current heating, the growth rate of the resistive wall instability, and single-bunch instability impedance limits.
\end{abstract}

"Operated by the Universities Research Association, Inc., for the U.S. Department of Energy under contract No. DE-AC3589ER40486. 
. 


\section{CONTENTS}

1. Introduction to Beam Tube Vacuum Issues in

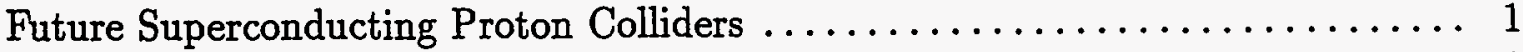

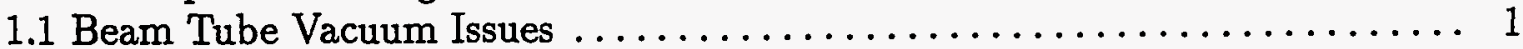

1.2 Synchrotron Radiation Parameters ......................... 2

1.3 Simulation in Electron/Positron Storage Rings .................. 3

1.4 Beam Tube Vacuum Requirements .......................... 5

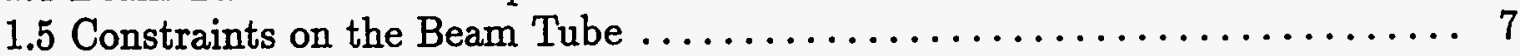

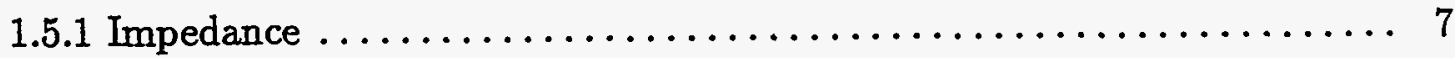

1.5.2 Beam Tube Resistivity Constraints $\ldots \ldots \ldots \ldots \ldots \ldots \ldots \ldots \ldots \ldots$

2. Model Equations for Vacuum in a Cryosorbing Beam Tube

Exposed to Synchrotron Radiation $\ldots \ldots \ldots \ldots \ldots \ldots \ldots \ldots \ldots \ldots \ldots, \ldots$

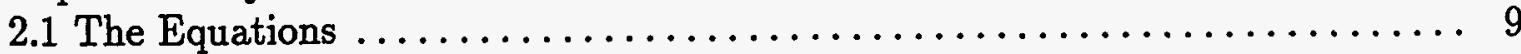

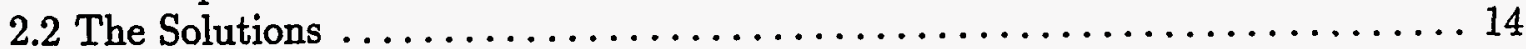

2.2.1 Short Beam Tube Without a Liner ..................... 14

2.2.2 Long 4.2-K Beam Tube Without a Liner .................. 16

2.2.3 Beam Tube Length Criterion, Axial Diffusion Times ........... 17

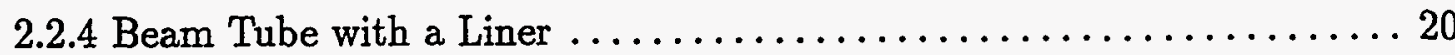

2.2.5 Model of Beam Tube Density at the Ends ................ 20

2.3 Ion Desorption ..................................... 22

3. Beam Tube Photodesorption Experiments ...................... 24

3.1 Room-Temperature Experiments . . . . . . . . . . . . . . . . . . . . . 24

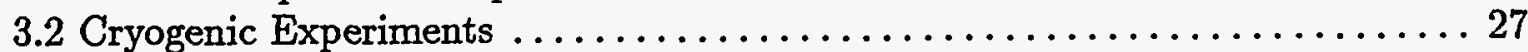

3.2.1 The CDG Experiments ............................ 27

3.2.2 The BINP Experiments ........................... 32

3.2.3 Comparison of CDG and BINP Experiments ................ 38

4. Some Related Vacuum Experiments $\ldots \ldots \ldots \ldots \ldots \ldots \ldots \ldots \ldots \ldots \ldots \ldots \ldots$

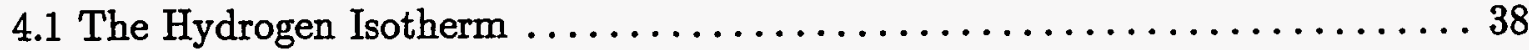

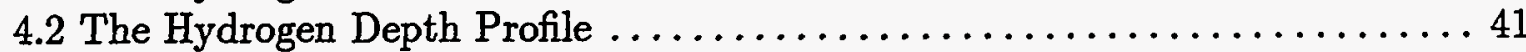

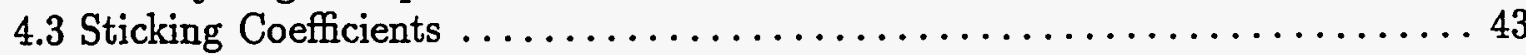

5. Liner Vacuum Conductance and Impedance $\ldots \ldots \ldots \ldots \ldots \ldots \ldots \ldots \ldots \ldots$

6. Model Vacuum Calculations ................................. 47

6.1 Simple Beam Tube $\ldots \ldots \ldots \ldots \ldots \ldots \ldots \ldots \ldots \ldots \ldots \ldots \ldots \ldots \ldots$

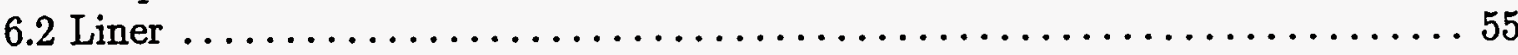

7. New Techniques for Measurement of Gas Density in a Cryosorbing Beam Tube . 58

$7.1 \mathrm{H}^{+} / \mathrm{H}^{-}$Ion Beam Method ............................... 58

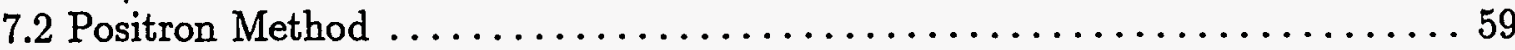




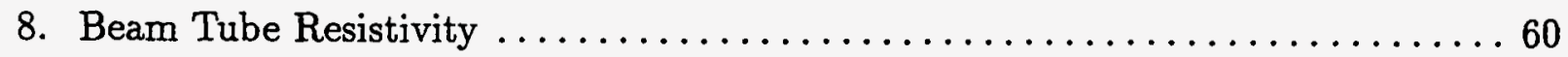

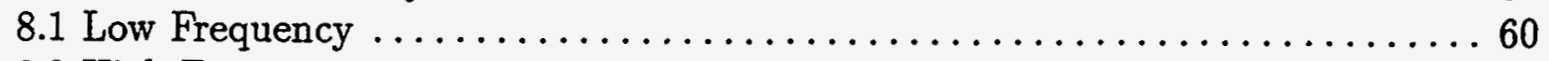

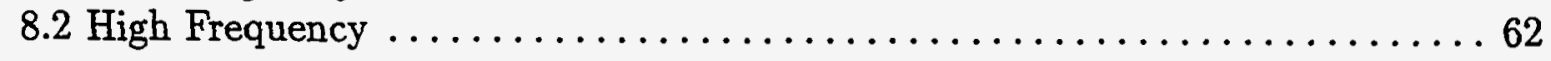

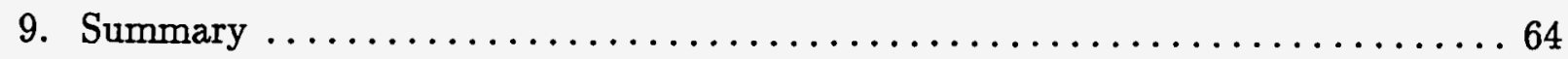

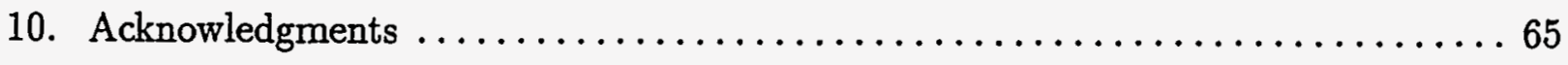

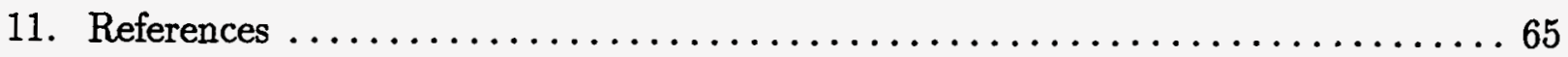

12. Appendix-Three-component Formulation of the Model Vacuum Equations . . . 68 


\section{Introduction to Beam Tube Vacuum Issues in Future Superconducting Proton Colliders}

\subsection{Beam Tube Vacuum Issues}

The future proton colliders that have been contemplated-the Superconducting Super Collider (SSC), ${ }^{1}$ Large Hadron Collider (LHC), ${ }^{2}$ and Eloisatron (ELN) ${ }^{3}$-encounter significant fluxes of synchrotron radiation inside the cold bore tube of superconducting magnets operating at LHe temperatures. For the accelerator designer this introduces three effects that need to be taken into account: the energy loss of the circulating protons, which must be made up by the rf system; the power deposited by the synchrotron radiation, which must be removed by cryogenic refrigeration; and the photodesorption of gas, which must be pumped by the beam tube vacuum system. Here we will be concerned with the third of these effects. The beam tube vacuum issues are indicated in simplified form in Figure 1. Circulating protons radiate photons as their orbits are bent by the superconducting dipole magnets. If the beam tube is chosen to coincide with the bore tube of the superconducting magnets, tightly bound $\mathrm{H}, \mathrm{C}$, and $\mathrm{O}$ in the near surface layer are converted by photodesorption into a steadily increasing surface density of physisorbed molecules (Figure 1(a)). The term "tightly bound" includes chemical binding and any other form of binding that is not readily desorbed by warming the tube to room temperature or less. Desorption by scattered photons is significant, so one has to contend with photodesorption from the entire circumference of the beam tube although the full width half maximum (FWHM) height of the strip of initial photon impact is the order of millimeters. Although beam tube pumping by physisorption is very high, the physisorbed molecules $\left(\mathrm{H}_{2}, \mathrm{CH}_{4}, \mathrm{CO}\right.$, and $\left.\mathrm{CO}_{2}\right)$ are weakly bound to the beam tube surface $\left(\sim 20 \mathrm{meV}\right.$ for $\left.\mathrm{H}_{2}\right)$ and themselves become an important source of photodesorption that builds up with time. In addition, the isotherm density of the physisorbed $\mathrm{H}_{2}$ also increases with time and may become significant, depending on the temperature of the beam tube. The source of molecular density inside a cryosorbing beam tube, then, has three components: (1) the photodesorption products of tightly bound $\mathrm{H}, \mathrm{C}$, and $\mathrm{O}$; (2) the photodesorbed, physisorbed molecules; and (3) the thermally desorbed isotherm density of physisorbed molecules (for $\mathrm{H}_{2}$ only, and He if there are leaks). The first of these would be expected to decrease with time as the surface becomes depleted of tightly bound $\mathrm{H}, \mathrm{C}$, and $\mathrm{O}$; however, the last two increase and if they become large enough would require shutdown of the accelerator, warm-up of the beam tube, and pumpout of the desorbed gases before resuming operations. Shutdowns that are too frequent reduce accelerator availability and the effectiveness of the physics program. The potential problems of the simple beam tubephotodesorption of physisorbed molecules and the $\mathrm{H}_{2}$ isotherm density-are alleviated by the liner concept in Figure 1(b). The liner is a coaxial, perforated tube fitting inside the magnet bore tube. Physisorbed molecules accumulate behind the liner and out of the view of the photons. The effective surface area of the magnet bore tube can be increased many times by the addition of cryosorbing material behind the liner, thus postponing increase of the $\mathrm{H}_{2}$ isotherm density. The liner also has the attractive feature of being operable at a temperature higher than the magnet cryostat, thus improving the efficiency of removing the synchrotron radiation heat load. The disadvantages of the liner are increases in cost and engineering complexity. A thorough 
understanding of photodesorption in a cryosorbing beam tube environment is needed to make a reasonable decision between a liner or no liner.

We recognize that the molecular form of the tightly bound $\mathrm{H}, \mathrm{C}$, and $\mathrm{O}$ in the surface layer of the beam tube is different from the $\mathrm{H}_{2}, \mathrm{CH}_{4}, \mathrm{CO}$, and $\mathrm{CO}_{2}$ that appear in the gas phase following photodesorption. For simplicity we will refer to the tightly bound $\mathrm{H}, \mathrm{C}$, and $\mathrm{O}$ and the immediate desorption products as "the tightly bound molecules" in the remainder of the report.
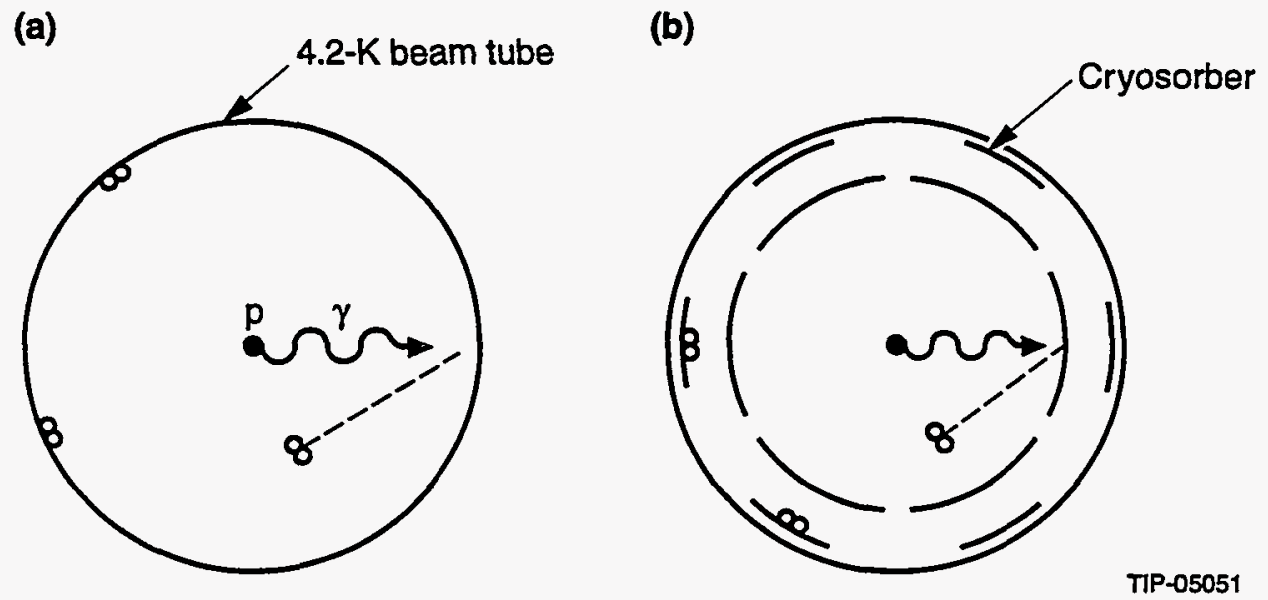

Figure 1. Schematic of simple beam tube (a) and liner (b) configurations.

\subsection{Synchrotron Radiation Parameters}

The synchrotron radiation photon flux $d \Gamma / d t$, critical energy $E_{\text {crit }}$, power per unit length $P /(2 \pi \rho)$, and FWHM angular width of radiated photons $\Delta \Psi$ are given by Eqs. (1)-(4) in convenient units, where $I(\mathrm{~mA})$ is the circulating proton beam current, $\rho(\mathrm{km})$ is the bending radius, $m_{p} c^{2} \gamma$ is the proton energy, and $E(\mathrm{eV})$ is the energy of a radiated photon. ${ }^{4}$ The synchrotron radiation power increases with the fourth power of $\gamma$; three powers of $\gamma$ are due to the hardening of the photon spectrum, and one power is due to the increase in intensity.

$$
\begin{gathered}
\left.\frac{d \Gamma}{d t} \text { (photons } / \mathrm{m} / \mathrm{s}\right)=6.54 \times 10^{10} \frac{I(\mathrm{~mA}) * \gamma}{\rho(\mathrm{km})} \\
E_{\text {crit }}(\mathrm{eV})=2.96 \times 10^{-10} \frac{\gamma^{3}}{\rho(\mathrm{km})} \\
\frac{P}{2 \pi \rho}(\mathrm{W} / \mathrm{m})=9.6 \times 10^{-19} \frac{\gamma^{4} I(\mathrm{~mA})}{\rho^{2}(\mathrm{~km})} \\
\Delta \Psi(\mathrm{rad})=\frac{2}{\gamma} *\left(\frac{E_{\text {crit }}}{E}\right)^{1 / 3}, E \ll E_{\text {crit }} .
\end{gathered}
$$


Half of the synchrotron radiation power is carried away by photons with energy $E>E_{\text {crit }}$. For some purposes it is useful to know the energy above which half of the photons are radiated-the median photon energy, $E_{1 / 2}$. This energy is given by

$$
E_{1 / 2}=0.08 * E_{\text {crit }} .
$$

In Table 1 we have listed the photon intensity, critical energy, and power radiated per unit orbit length for the future colliders and the existing 1-TeV Tevatron at Fermi National Accelerator Laboratory (FNAL) in the United States. Passing from the Tevatron to LHC and SSC, the critical energy increases from the infrared region, where photodesorption coefficients have negligible magnitude, to the soft $\mathrm{x}$ ray region, where initial photodesorption coefficients on technical metal surfaces have magnitude $\sim 10^{-2}$ molecules/photon. Furthermore, the photon fluxes have increased to levels where the magnitude of photodesorbed gas will require thorough understanding to be certain that the vacuum design goals required by luminosity can be met. The synchrotron radiation heat loads have also increased to levels that are significant fractions of the heat leak budget required for superconducting magnets. For the LHC, which has a magnet cryostat temperature $1.9 \mathrm{~K}$, it is not practical to allow the synchrotron radiation to fall on the magnet bore tube, and a warm liner $(4.2-20 \mathrm{~K})$ is required for removing the radiation heat load. For the SSC, the magnet cryostat temperature is $4.2 \mathrm{~K}$, and it is still practical to remove the heat on the magnet bore tube. Going further to the ELN-which, it must be admitted, is an imaginary machine at this point-the critical energy is in the hard $\mathrm{x}$ ray region, and the magnitude of radiation heat load is much greater than the refrigeration capacity required by superconducting magnets. Synchrotron radiation in ELN would clearly play a major design role, and a warm liner would be required for removing the heat load.

Table 1. Synchrotron radiation parameters of superconducting proton colliders.

\begin{tabular}{|l|c|c|c|c|}
\hline & Tevatron & LHC & SSC & ELN \\
\hline$E(\mathrm{TeV})$ & 1 & 7.0 & 20 & 100 \\
\hline$I(\mathrm{~mA})$ & 5 & 530 & 72 & 400 \\
\hline$\rho(\mathrm{km})$ & 0.76 & 2.7 & 10.1 & 43.3 \\
\hline$B(\mathrm{~T})$ & 4.4 & 8.65 & 6.6 & 7.7 \\
\hline$d \Gamma / d t^{*}($ photons $/ \mathrm{m} / \mathrm{s})$ & $4.5 \times 10^{14}$ & $9.6 \times 10^{16}$ & $1 \times 10^{16}$ & $6.5 \times 10^{16}$ \\
\hline$E_{\text {crit }}(\mathrm{eV})$ & 0.5 & 46 & 284 & 8400 \\
\hline$P / 2 \pi \rho(\mathrm{W} / \mathrm{m})^{*}$ & $10^{-5}$ & 0.22 & 0.14 & 27 \\
\hline
\end{tabular}

${ }^{*}$ per beam

\subsection{Simulation in Electron/Positron Storage Rings}

Since the pioneering experiment described in Reference 4, whenever photodesorption is expected to play a major role in a future accelerator it has become customary to simulate the accelerator beam tube vacuum conditions in an external white light beamline at an electron (or positron) synchrotron radiation light source. The basic idea is illustrated in Figure 2. A length of beam tube $l_{t}$ is placed a distance $l_{s}$ from a tangent to the electron orbit in a bending magnet. 
The beam tube is tilted at an angle $\delta$ so that synchrotron radiation emitted over an angle $\Delta \alpha$ of the electron beam orbit is intercepted by the beam tube. The angles $\delta$ and $\Delta \alpha$ are related by

$$
\Delta \alpha=\frac{\delta * l_{t}}{2}\left(\frac{1}{l_{s}}+\frac{1}{l_{s}+l_{t}}\right)
$$

and the photon intensity striking the tube is given by

$$
\begin{aligned}
\frac{d \Gamma}{d t}(\text { photons } / \mathrm{m} / \mathrm{s}) & =6.54 \times 10^{13} \frac{I(\mathrm{~mA}) \gamma \Delta \alpha}{l_{t}(\mathrm{~m})} \\
& =3.27 \times 10^{13} I(\mathrm{~mA}) \gamma \delta\left(\frac{1}{l_{s}}+\frac{1}{l_{s}+l_{t}}\right) .
\end{aligned}
$$

(a)

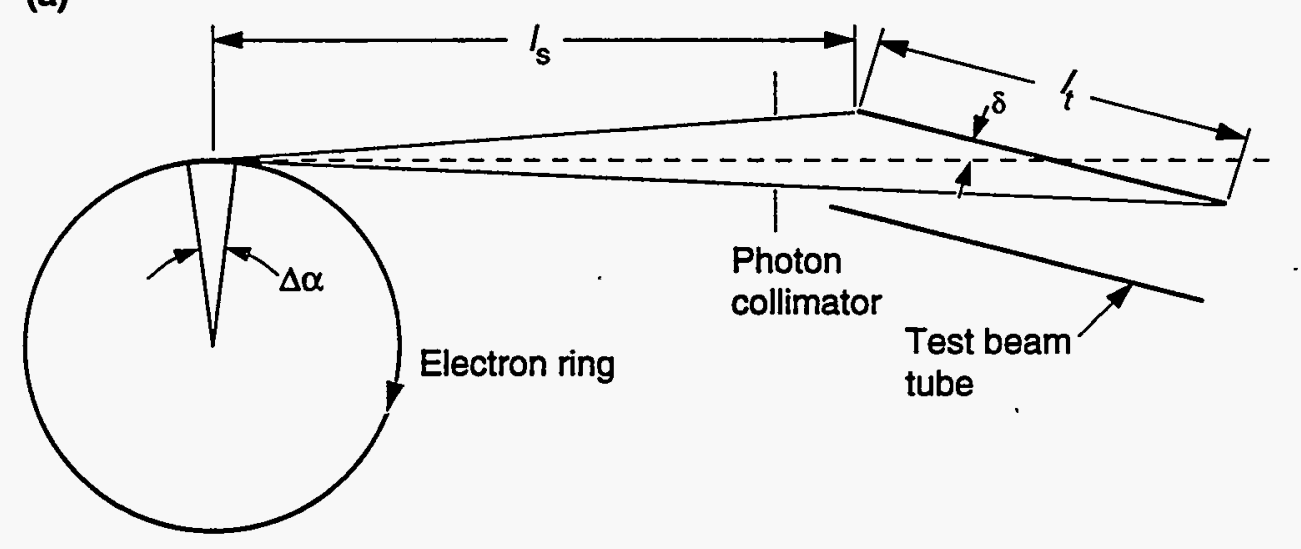

(b)

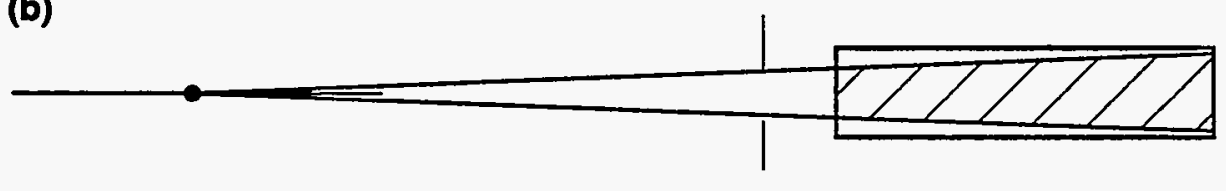

TIP-05721

Figure 2. Schematic of the illumination of a beam tube in an external white light beamline at an electron synchrotron radiation light source: (a) horizontal, (b) vertical.

In Eq. (7) we have assumed that the loss of photons due to vertical collimation is negligible; this needs to be checked and possibly corrected for in the actual experiments. The ratio $\frac{\gamma^{3}}{\rho} \sim \frac{\gamma^{2} B}{m}$ is chosen to match the future accelerator so the critical energy and, therefore, the photon spectrum is reproduced. For the proton colliders LHC and SSC the photon spectrum is reproduced by UV electron storage rings with beam energy $\sim 500 \mathrm{MeV}$. In practice it isn't practical to match all the details of a future proton machine at an electron light source. Because of lower $\gamma$ in the electron rings, the angular spreading of the synchrotron radiation is increased 
(Eq. (4)), and the vertical height of the strip directly illuminated by photons is increased. At low energy some photons may be cut off on the collimators and not reach the beam tube. In addition, in order to achieve adequate photon intensity, the angle of incidence, $\delta$, is increased in the electron ring experiments compared to the proton machine. This is particularly important for obtaining long-exposure information, where one would like to compress a year of operation at a proton collider into a few weeks' data run at an electron light source. Generally these are not regarded as serious limitations. For example, illuminating a larger area of the beam tube in the simulation experiments would tend to lead to a slower clean-up time compared to the actual proton collider, and therefore to a conservative vacuum design. This situation is also ameliorated by diffuse photon scattering followed by photodesorption. The dependence of the photodesorption coefficient on angle of incidence has been observed to be fairly weak below 20 mrad, ${ }^{4}$ probably because of the roughness of the technical metal surfaces used for beam tubes.

\subsection{Beam Tube Vacuum Requirements}

The vacuum requirements for a superconducting proton collider are derived by considering the effect of beam gas interactions on: (1) the loss of luminosity, and (2) the scattered beam power deposited in the cold mass of the cryostats. The first leads to a limit on the circumferentially averaged gas density. The second leads to a limit on local gas density. For the SSC the goal for vacuum-limited luminosity lifetime is $150 \mathrm{~h}$. The vacuum-limited luminosity lifetime $\tau_{L, \text { vac }}$ is given by a sum over species of circumferentially averaged gas densities $\bar{n}_{j}$ :

$$
\frac{1}{\tau_{L, \mathrm{vac}}}=2 \sum_{j} \bar{n}_{j} \sigma_{p j} c
$$

where $\sigma_{p j}$ is the total proton cross section on species $j$, which leads to a proton lost from the beam, including inelastic and elastic scattering, and $c$ is the velocity of light. These cross sections are tabulated in Table 2 for the most important photodesorbed molecules. Although $\mathrm{H}_{2}$ is generally the dominant gas desorbed, one must pay attention to the others because of their larger cross sections. For example, the cross sections for $\mathrm{CO}$ and $\mathrm{CO}_{2}$ are an order of magnitude larger than for $\mathrm{H}_{2}$, so the photodesorption coefficients need to be ten times less in order for them not to dominate. In fact, it will turn out that in a liner configuration, beam gas scattering by $\mathrm{CO}$ dominates $\mathrm{H}_{2}$. This could be true for $\mathrm{CO}_{2}$ as well, but the photodesorption coefficient of $\mathrm{CO}_{2}$ at LHe temperatures has not yet been determined. As can be seen from Table 2 the cross sections do not depend strongly on energy, so the gas density bounds for a given luminosity lifetime will be similar for all the machines we are considering. Bounds on circumferentially averaged gas density for $150-\mathrm{h}$ luminosity lifetime and $20-\mathrm{TeV}$ beam energy are given in the second column of Table 3 for $\mathrm{H}_{2}, \mathrm{CH}_{4}, \mathrm{H}_{2} \mathrm{O}, \mathrm{CO}$, and $\mathrm{CO}_{2}$ taken separately. 
Table 2. Beam gas scattering cross sections.*

\begin{tabular}{|l|c|c|c|}
\hline & $7 \mathrm{TeV}$ & $20 \mathrm{TeV}$ & $100 \mathrm{TeV}$ \\
\hline $\mathrm{H}$ & $50 \mathrm{mb}$ & 52 & 60 \\
\hline $\mathrm{C}$ & 400 & 420 & 444 \\
\hline $\mathrm{O}$ & 410 & 540 & 570 \\
\hline $\mathrm{H}_{2}$ & 100 & 104 & 120 \\
\hline $\mathrm{CH}_{4}$ & 600 & 628 & 648 \\
\hline $\mathrm{H}_{2} \mathrm{O}$ & 510 & 644 & 690 \\
\hline $\mathrm{CO}$ & 810 & 960 & 1014 \\
\hline $\mathrm{CO}_{2}$ & 1220 & 1500 & 1584 \\
\hline
\end{tabular}

* The data in this table were kindly supplied by Nikolai Mokhov.

Table 3. Average and local gas density bounds for the 20-TeV SSC.

\begin{tabular}{|l|c|c|}
\hline & average $^{a}$ & local $^{b}$ \\
\hline $\mathrm{H}_{2}$ & $3.0 \times 10^{8} / \mathrm{cm}^{3}$ & $4.0 \times 10^{10}$ \\
\hline $\mathrm{CH}_{4}$ & $5.0 \times 10^{7}$ & $6.6 \times 10^{9}$ \\
\hline $\mathrm{H}_{2} \mathrm{O}$ & $4.8 \times 10^{7}$ & $6.5 \times 10^{9}$ \\
\hline $\mathrm{CO}$ & $3.3 \times 10^{7}$ & $4.3 \times 10^{9}$ \\
\hline $\mathrm{CO}_{2}$ & $2.1 \times 10^{7}$ & $2.8 \times 10^{9}$ \\
\hline
\end{tabular}

The locally scattered beam power per unit length, $d P_{\text {scatt }} / d z$, is given by a similar sum over local gas densities $n_{j}$ :

$$
\frac{d P_{\text {scatt }}}{d z}=I_{b} E_{b} \sum_{j} n_{j} \sigma_{p j}
$$

where $E_{b}$ is the proton beam energy and $I_{b}$ is the beam current. Since most of the products from a single beam gas interaction are deposited over a downstream path length of $\sim 40 \mathrm{~m}$, we are imagining a local gas density bump extending over roughly this scale length or greater. Greater than $90 \%$ of the beam gas scattered power is deposited in the cold mass of the magnet cryostats, where it leads to an increase in coil temperature and can initiate a magnet quench if the increase is too large. Figure 3 shows the results of an analysis for the SSC. ${ }^{5} \mathrm{~A}$ local pressure bump of $4 \times 10^{10} \mathrm{H}_{2} / \mathrm{cm}^{3}$ in the ninth dipole of a cell, corresponding to $0.6 \mathrm{~W} / \mathrm{m}$ of scattered beam power, is estimated to cause a $\sim 100-\mathrm{mK}$ temperature increase in the LHe and $\mathrm{a} \sim 500-\mathrm{mK}$ increase in the superconducting coil of the tenth dipole. Since the temperature margin specified for the SSC dipoles is $600 \mathrm{mK}$, we take $0.6 \mathrm{~W} / \mathrm{m}$ as a working limit on scattered beam power, and the corresponding local limits on gas density are given in the third column of Table 3, again taken separately for each species. In practice, a gas mixture will be present and the total $\mathrm{H}_{2}$ equivalent density of all species will have to be less than the bounds given in the 
first line of Table 3 . The main variation in the local gas density bound from machine to machine will be due to different beam current $I_{b}$ and energy $E_{b}$. The usual case is that the average density bound, given by Eq. (8), is considerably less than the local bound given by Eq. (9). In principle it is possible that as the beam current and energy increase, the local bound due to energy deposited in the magnet cryostats will become more restrictive than the bound due to luminosity lifetime. However, even the 100-TeV ELN has not reached this situation, assuming that the limiting scattered power density will not be less than the specification taken for the $\mathrm{SSC}, \sim 0.6 \mathrm{~W} / \mathrm{m}$.
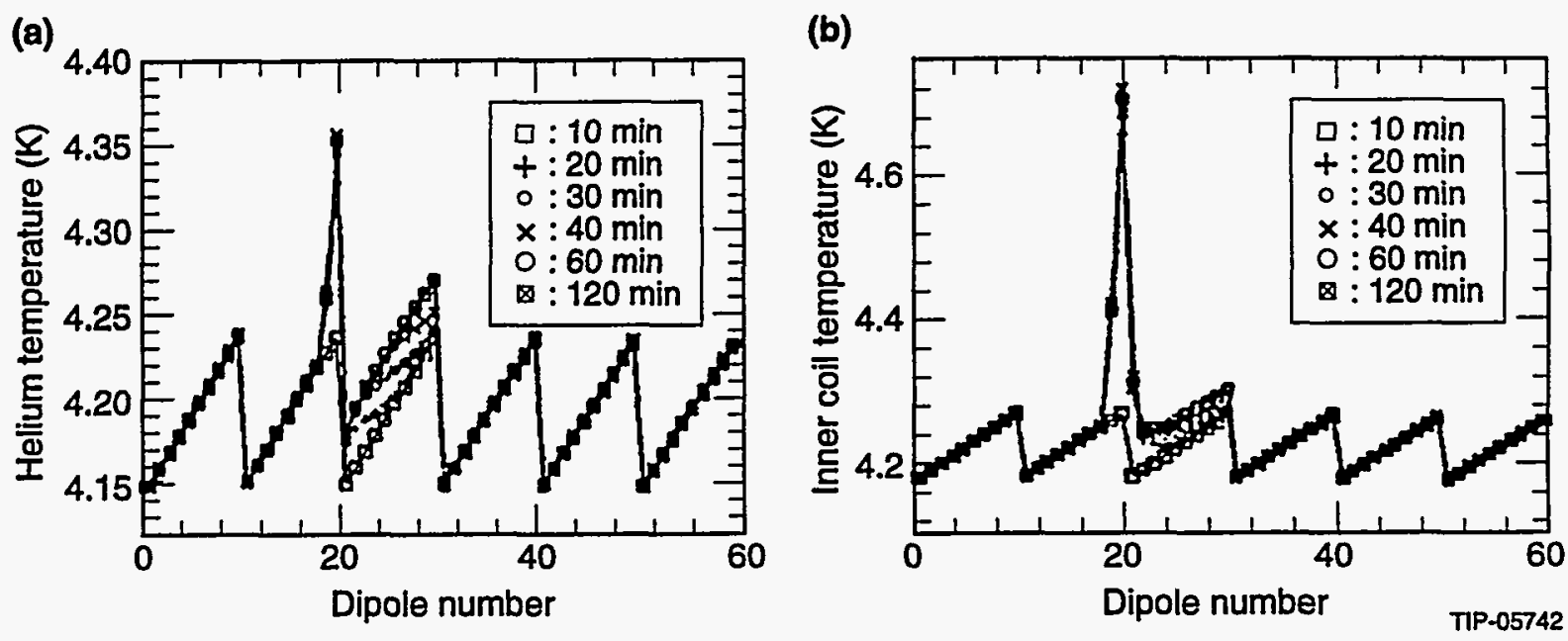

Figure 3. Temperature profiles along a 1-km section for a $4 \times 10^{10} \mathrm{H}_{2} / \mathrm{cm}^{3}$ pressure bump: (a) helium, (b) inner coil. ${ }^{5}$

\subsection{Constraints on the Beam Tube}

In addition to the vacuum requirements, the beam tube is constrained by impedance and surface resistivity considerations. The origins of these constraints are discussed in this section, and numerical results are given in Sections 5 and 8.

\subsubsection{Impedance}

The impedance of all the accelerator structures seen by the circulating beam, including the beam tube and liner perforations, must be less than the threshold impedance of the most unstable single bunch instability. The impedance thresholds and estimates are dependent on the details of a particular accelerator. For the case of the SSC the instabilities with the lowest thresholds are the transverse mode coupling instability (or fast head-tail instability) for the transverse impedance and the microwave instability for the longitudinal impedance. ${ }^{1}$ The most recent estimates of the threshold impedances for the SSC design parameters have been given by W. Chou: $\operatorname{Im}\left(Z_{T}\right)=170 \mathrm{M} \Omega / \mathrm{m}$, and $\left|Z_{L} / n\right|=4.1 \Omega .^{6}$ These impedance thresholds increase linearly with proton energy and are inversely proportional to the number of protons in a bunch. In addition, Chou has given an estimate of the impedances of all the accelerator structures 
(bellows, beam position monitors, etc.) in the SSC. For the transverse impedance, excluding a liner, Chou's results can be fit with $\operatorname{Im} Z_{T, 0}(\mathrm{M} \Omega / \mathrm{m})=23.8(16 / a(\mathrm{~mm}))^{2.6}$, where $a$ is the radius of the beam tube. The longitudinal impedance is only weakly dependent on $a$, and Chou's result is $\frac{Z_{L, 0}}{n}(\Omega)=0.34$. For a beam tube radius, $a=16 \mathrm{~mm}$, the ratios of threshold to estimated impedances are $\sim 170 / 23.8=7$ in the transverse case and $\sim 4.1 / 0.34=12$ in the longitudinal case. These ratios allow some room for impedance increase due to a liner; however, one would like to preserve as much safety margin as possible to allow for uncertainty in the impedance estimates and operation at upgraded beam intensity. The impedances of perforations in the liner are discussed in Section 5.

\subsubsection{Beam Tube Resistivity Constraints}

Constraints on beam tube resistivity are derived at low frequency (of the order of the fractional betatron tune and less than $1 \mathrm{kHz}$ ) from the growth time for the multibunch resistive wall instability, which must be stabilized by feedback. At high frequency (of the order of the bunch length frequency or in the $\mathrm{GHz}$ range) the beam tube resistivity is constrained by image current heating of the wall. For the machines we are considering, adequately long resistive wall growth time and low image current heating can be achieved by only copper or aluminum. At low frequencies of the order of the fractional betatron tune, the skin depth is large compared to the thickness of copper on the inside of the beam tube, and there are corrections due to the conductivity of the outer layers., ${ }^{7,8}$ However, for the cases we will examine it is a good approximation (i.e., accurate to $\sim 1 \%$ in the growth time) to neglect the outer layers of stainless steel and keep only the leading term in the ratio of copper thickness to skin depth. The resulting expression for the growth rate is

$$
\tau_{r w}=\pi\left(\frac{a^{3}}{M N_{b} r_{p} \bar{\beta} c}\right)(\gamma \Delta \nu)\left(Z_{0} \sigma_{c} t\right),
$$

where $a$ is the beam tube radius, $N_{b}$ the number of protons per bunch, $M$ the number of bunches, $r_{p}$ the classical proton radius, $\bar{\beta}$ the average value of the lattice betatron function, $\Delta \nu$ the fractional betatron tune, $Z_{0}$ the impedance of free space, $\sigma_{c}$ the copper conductivity, and $t$ the copper thickness. We note that the dependence on the high-conductivity layer appears as the product of conductivity and thickness, as one would expect at low frequency. Data on low-frequency conductivity at the beam tube operating temperature and including the effect of magnetic field are required to obtain numerical estimates of $\tau_{r w}$. The resistive wall growth time depends linearly on the beam energy factor $\gamma$, so requirements for feedback stabilization are most demanding at injection energy. The growth time increases strongly as the third power of the beam tube radius. Two factors will make stabilization of the resistive wall instability more difficult as the machine size and luminosity increase: the inverse dependence of $\tau_{r w}$ on the product of bunch number and protons per bunch, and the increase in machine circumference. These are partially offset by the increase in injection energy. Numerical estimates of $\tau_{r w}$ are given in Section 8.1. 
At high frequencies, of the order of the bunch length frequency $f \approx \frac{1}{2 \pi} \frac{c}{\sigma_{z}} \approx 1 \mathrm{GHz}$, where $\sigma_{z}$ is the rms bunch length, the high-conductivity layer required by the resistive wall instability is thick compared to the skin depth. The heating of the beam tube wall by the beam image current may then be written $a^{9}$

$$
P=\Gamma\left(\frac{3}{4}\right) M\left(\frac{N_{B} e c}{2 \pi}\right)^{2}\left(\frac{Z_{0} \rho}{2 \sigma_{z}}\right)^{1 / 2} \frac{1}{\sigma_{z} a}
$$

where $\rho$ is the resistivity of the surface material. Experiments in the range of the bunch frequency measure the surface resistance $R_{s}=\frac{\rho}{\delta}$, where the skin depth $\delta=\left(\frac{2 \rho}{\mu_{0} \omega}\right)^{1 / 2}$. If we substitute for the frequency $\omega \approx \frac{c}{\sigma_{z}}$, then Eq. (11) can be written in terms of the surface resistance, $R_{s}$ :

$$
P \approx \Gamma\left(\frac{3}{4}\right) M\left(\frac{N_{B} e c}{2 \pi}\right)^{2}\left(\frac{R_{s}}{\sigma_{z} a}\right) .
$$

Numerical estimates of the magnitude of beam image current heating are given in Section 8.2, together with discussion of the available data measuring $R_{s}$.

\section{Model Equations for Vacuum in a Cryosorbing Beam Tube Exposed to Synchrotron Radiation}

\subsection{The Equations}

We begin by writing down equations describing the volume density $n$ and surface density $s$ of $\mathrm{H}_{2}$ inside a cryosorbing beam tube of radius $a$ exposed to synchrotron radiation. ${ }^{10}$ Some comments on the treatment of other gases $\left(\mathrm{CO}, \mathrm{CO}_{2}, \mathrm{CH}_{4}\right)$ will be made later. Throughout this report we consistently refer to the density inside a beam tube and avoid the use of pressure. This may cause some discomfort for the hardcore vacuum person, but there are two good reasons for this: it avoids confusion over the temperature at which the pressure is given, and accelerator requirements are naturally given in terms of beam tube density. The equation for volume density is

$$
V \frac{\partial n}{\partial t}=\eta_{1} \dot{\Gamma}+\eta^{\prime} \dot{\Gamma}+\frac{A_{w} s}{\tau_{w}^{t}}-\sigma_{w} S_{w} * n-C * n+A_{c} D \frac{\partial^{2} n}{\partial z^{2}} .
$$

In Eq. (13) $V=\pi a^{2}$ is the beam tube volume per unit axial length. The first two terms on the right describe photodesorption of molecules. The desorption coefficient $\eta_{1}$ molecules per photon is for tightly bound hydrogen not previously desorbed and is a function of the integrated photon flux per unit length $\Gamma$ and perhaps the photon intensity $\dot{\Gamma}$. The second term allows for the desorption of relatively weakly-bound physisorbed molecules with desorption coefficient $\eta^{\prime}$, which is a function of the surface density $s$ of physisorbed molecules. At low surface coverage one would expect $\eta^{\prime}$ to depend linearly on the surface density $s ; \eta^{\prime}=\eta_{0}^{\prime}\left(\frac{s}{s_{m}}\right)$, where $s$ has been normalized to the monolayer density $s_{m}$. The third term is the thermal desorption rate of physisorbed molecules with sojourn time $\tau_{w}^{t}$. The fourth term on the right of Eq. (13) accounts for the cryosorption of molecules incident on the surface; $\sigma_{w}$ is the sticking probability per 
wall collision, $S_{w}=\bar{v} A_{w} / 4$ is the ideal wall pumping speed, $\bar{v}$ is the mean molecular speed, and $A_{w}=2 \pi a$ is the beam tube wall area per unit axial length. The fifth term allows for a liner or distributed pump with pumping speed $C$ per unit axial length. In the case of a liner with $C \neq 0$ we have neglected the back-streaming of molecules from the outer coaxial region. Because the conductance of the holes in the liner is to be chosen small compared to the pumping speeds of the magnet bore tube surface and cryosorber in the outer annular region, this is a good approximation. A two-region vacuum model of an $80-\mathrm{K}$ liner that retains the back-streaming term is discussed in Reference 11. The last term in Eq. (13) allows for the axial diffusion of molecules out the ends of a finite-length tube; $A_{c}=\pi a^{2}$ is the tube cross section area and $D=\frac{2}{3} a \bar{v}$ is the Knudsen diffusion coefficient.

The equation for the surface density $s$ of cryosorbed molecules is

$$
A_{w} \frac{\partial s}{\partial t}=\eta_{2} \dot{\Gamma}+\sigma_{w} S_{w} * n-\frac{A_{w} s}{\tau_{w}^{t}}-\eta^{\prime} \dot{\Gamma} .
$$

The first term in Eq. (14) allows for the possibility of direct production of physisorbed molecules by the incident photon flux, $\eta_{2}$ molecules per photon. The second, third, and fourth terms are the same as in Eq. (13) but with opposite sign. The last three terms on the right of Eq. (14) define the effective pumping of the beam tube surface, which we note is reduced by thermal desorption and by the photodesorption or recycling of cryosorbed molecules. In Eq. (14) we neglect the surface mobility of physisorbed molecules, so axial transport occurs only through gas phase diffusion described by Eq. (13).

One more equation is needed to describe the equilibrium isotherm relating the equilibrium vapor pressure $n_{e}$ due to thermal evaporation to the surface density $s$. Here we choose to represent the isotherm by a Brunauer, Emmett, Teller (BET) equation: ${ }^{12}$

$$
x=\frac{\alpha y}{[(1-y)(1+(\alpha-1) y)]},
$$

where $x=s / s_{m}, y=n_{e} / n_{\text {sat }}$, and $\alpha=e^{\theta / T}$ is a dimensionless parameter where $\theta$ is the difference between the molecular adsorption energies of the first monolayer and the bulk adsorbate. Equation (15) has the saturation property $y \rightarrow 1$ as $x \rightarrow \infty$. The temperature dependence of the saturation density for $\mathrm{H}_{2}$ has been measured by Benvenuti et al. ${ }^{13}$ and found to be $n_{\text {sat }}\left(\mathrm{cm}^{-3}\right)=6.84 \times 10^{22} e^{-95.8 / T} / \mathrm{T}$. For any particular surface the equilibrium isotherm must be measured experimentally to determine $s_{m}$ and $\alpha$. An example for the electrodeposited $\mathrm{Cu}$ used in 4.2-K photodesorption experiments at Budker Institute of Nuclear Physics (BINP) will be given in Section 4.1. The isotherm density is most sensitive to $\alpha$ for $x \ll 1, y \ll 1$, in which case $x=\alpha y$. However, this region is difficult to access experimentally because the isotherm density is very low and can be masked by desorption due to thermal radiation leaking in from warm regions of the vacuum system or by diffusion from the bulk materials of the ionization gage or residual gas analyzer (RGA) used for pressure measurement. Some progress in this direction has recently been made by M.G. Rao at the Continuous Electron Beam Accelerating Facility (CEBAF) by cooling the walls of an extraction gage to LHe temperature. A background $\mathrm{H}_{2}$ density $<10^{6} / \mathrm{cm}^{3}$ has been achieved by this method. ${ }^{14}$ For our purposes $s_{m}$, which depends 
on the particular surface, and $n_{\text {sat }}$ are the most important parameters, and results are not too sensitive to uncertainty in $\alpha$. Equation (15) is, of course, an approximate representation of a real isotherm, and for some purposes it may be desirable to use a more complex expression or interpolation of actual measured data. An additional complexity is the reduction of the $\mathrm{H}_{2}$ isotherm density over an adsorbed gas mixture that appears in the data of $\mathrm{E}$. Wallen at CERN. ${ }^{15}$ The reduction is small for a few percent $\mathrm{CO}$ added to $\mathrm{H}_{2}$, but becomes noticeable for a 25\% CO admixture. Equation (15), however, exhibits the main physical effects of thermal desorption-the rapid rise in vapor pressure as one monolayer is approached and saturation as a thick surface layer builds up-and is useful for modeling calculations.

If detailed balance is applied to the $\mathrm{H}_{2}$ isotherm, $\sigma_{w}^{t} \frac{A_{w} \bar{v}_{t}}{4} n_{e}=\frac{A_{w} s}{\tau_{w}^{t}}$, and we can invert the isotherm Eq. (15) to obtain the following relations for the thermal sojourn time:

$$
\begin{aligned}
\tau_{w}^{t}(s) & =\frac{4}{\sigma_{w}^{t} \bar{v}_{t}} \frac{s}{n_{e}} \\
& =\frac{4}{\sigma_{w}^{t} \bar{v}_{t}}\left(\frac{s_{m}}{n_{\text {gat }}}\right) \frac{2 \alpha x^{2}}{\sqrt{\alpha^{2}(1-x)^{2}+4 \alpha x}-(\alpha(1-x)+2 x)},
\end{aligned}
$$

where $x=s / s_{m}$ is the normalized density of physisorbed $\mathrm{H}_{2}$. In Eq. (16) $\sigma_{w}^{t}$ is the sticking coefficient of thermally desorbed gas-phase molecules corresponding to the equilibrium isotherm, and $\bar{v}_{t}$ is the mean speed of these molecules that have the temperature of the wall. The real utility of Eq. (16) is that it allows the thermal sojourn time to be calculated in systems that aren't closed and in equilibrium as long as the surface density of physisorbed $\mathrm{H}_{2}$ is known. In particular, we are interested in situations where there is an imbalance between thermal molecules sticking to the surface and those thermally desorbing, and Eq. (16) still gives a valid expression for the mean thermal desorption time as long as $s / s_{m}$ is known. Equation (16) is then useful for the integration of Eqs. (13) and (14) and also for estimating the mean time a cryosorbed molecule sticks to the wall before being thermally desorbed. Four limiting forms can be given for $\tau_{w}^{t}$ :

$$
\begin{array}{ll}
\tau_{w}^{t}=\frac{4}{\sigma_{w}^{t} \bar{v}_{t}}\left(\frac{s_{m}}{n_{\text {sat }}}\right) \alpha \quad \text { for } \quad \alpha y \ll 1, \\
\tau_{w}^{t}=\frac{4}{\sigma_{w}^{t} \bar{v}_{t}}\left(\frac{s_{m}}{n_{\text {sat }}}\right) \alpha^{1 / 2} & \text { for } \quad x=1, y \approx \frac{1}{\alpha^{1 / 2}}, \\
\tau_{w}^{t}=\frac{4}{\sigma_{w}^{t} \bar{v}_{t}}\left(\frac{s_{m}}{n_{\text {sat }}}\right)(4) & \text { for } \quad x=2, y \approx \frac{1}{2}, \text { and } \\
\tau_{w}^{t}=\frac{4}{\sigma_{w}^{t} \bar{v}_{t}}\left(\frac{s_{m}}{n_{\text {sat }}}\right) x & \text { for } \quad y \rightarrow 1 .
\end{array}
$$

We have assumed $\alpha=e^{\theta / T} \gg 1$ which is a good approximation for a surface at LHe temperature. The thermal sojourn time starts out at a maximum value at very low surface coverage, decreases rapidly near one monolayer, reaches a broad minimum at two monolayers, and then increases 
slowly as a thick layer of cryosorbed molecules builds up. The rapid decrease is due to the transition from the bonding energy of the first monolayer to the bonding energy of the bulk adsorbate. For a thick layer the thermal sojourn time increases linearly with the number of monolayers as it must, since the saturation vapor pressure is constant and only the top monolayer is evaporating. The first expression in Eq. (17) predicts that the sojourn time is independent of the surface density and isotherm vapor density in the low-coverage limit $\alpha y \ll 1$, which is the regime of most interest for a storage ring beam tube.

Equations (13) and (14) have been written for the total molecular density $n$, which is a sum of photodesorbed molecules that were tightly bound, photodesorbed molecules that were physisorbed, and thermally desorbed molecules. Alternatively we could have written a set of volume density equations for each of these three components, distinguishing between their mean speeds and sticking coefficients. We prefer to deal with the single equation (13), which is a sum of the three component equations with density-averaged mean wall pumping speed, $\sigma_{w} S_{w}$, diffusion coefficient $D$, and liner conductance $C$. No physical insight is lost and additional subscripts are avoided. In practice, except for relatively isolated time intervals, the density will be dominated by one of the three components, and it will be clear what to use for the mean values of $\sigma_{w} S_{w}, C$, and $D$. Developing an understanding of when each of the components is dominant will emerge from analysis of the solutions to these equations and the insight gained from experiments. Appendix A contains a short discussion of the equivalence of the single equation and the three-component equation approaches.

When the formulation given here has been described in the past, the motivation for treating separately the photodesorption of tightly bound molecules and the photodesorption of physisorbed molecules has sometimes been questioned. The argument is that a molecule is a molecule, and molecules are indistinguishable once desorbed. So how does one tell the difference? We will see from the analysis and also the experiments that have been done that situations dominated by the desorption of tightly bound and physisorbed molecules can be created, and they are dramatically different. The coefficient $\eta^{\prime}$ for photodesorption of physisorbed gas was introduced to explain the observation that the $\mathrm{H}_{2}$ gas density in a cryosorbing beam tube exposed to synchrotron radiation increases with time, in contrast to the gas density in warm beam tubes and open samples at LHe temperatures, which decreases with time. This increase was first observed in the cold-tube experiments of Bintinger et al. during the Central Design Group (CDG) era of the SSC. ${ }^{16,17}$

The equations given above have been written in terms of "white light" synchrotron radiation photon flux per unit length of beam tube, which is the universal practice with beam tube photodesorption experiments. From a fundamental viewpoint one would prefer to deal with a photon flux per unit area and a locally defined desorption coefficient, which is a function of photon energy, angle, integrated flux, and intensity. However, the precise details of the illumination of the surface of a beam tube exposed to synchrotron radiation are limited at this point. On the one hand only a fraction of the circumference of a beam tube is illuminated by direct synchrotron radiation-a strip with FWHM of the order of $\mathrm{mm}$ in the storage rings in Table 1 . On the other hand diffusely scattered radiation plays an important role; the side of the beam tube opposite direct illumination has been observed to have undergone significant desorption in several experiments where the beam tube has been rotated after long exposure to one side. ${ }^{18-20}$ In addition, the experiments in Reference 18 used an array of probes to observe the photoelec- 
tric current produced by the scattered photons. In a recent room-temperature experiment the conditioning of the surface opposite direct illumination appeared to be equivalent to $25 \%$ of the incident primary synchrotron radiation. ${ }^{20}$ Because of these complications it is not possible to extract fundamental quantities from beam tube photodesorption experiments. Rather the intent is to model closely enough the situation expected to be encountered so that one can predict what will occur in an actual proton collider. As an alternative one could attempt to do photodesorption experiments with small samples and well-defined photon flux, and proceed to measure the desorption coefficient as a function of photon energy, angle of incidence, intensity, and integrated flux. The photon flux in a beam tube exposed to synchrotron radiation would also have to be measured as a function of energy and angle and position, including the effects of scattering. The fundamental photodesorption coefficients and photon source function could then be integrated to predict the gas density in a beam tube. At the present time such an approach seems Herculean, and in any case would have to be followed by the type of experiment described here for one to be confident of the results.

The chemical form of the photodesorbed molecules in the beam tube material and the precise mechanism of photodesorption are not known in detail. $\mathrm{H}, \mathrm{C}$, and $\mathrm{O}$ atoms are present in the oxide layer on metal surfaces, and $\mathrm{H}$ atoms can diffuse from the bulk material as well. Since the earliest days of investigation it has been supposed that the photodesorption process takes place through production of a photoelectron that then breaks chemical bonds in some way. ${ }^{21}$ Recent viewpoints as expressed by Grobner et al. are similar: photons or photoelectrons dissociate molecules in the near surface layer; in a second step the atoms then diffuse and combine into $\mathrm{H}_{2}, \mathrm{CO}$, etc., which thermally desorb. ${ }^{22}$ In the experiment of Gomez-Goni et al., the pressure increase in a beam tube exposed to synchrotron radiation has been found to be a linear function of the secondary electron current emitted from the beam tube wall, supporting the idea that secondary electrons play a strong role in photodesorption. ${ }^{23}$ However, one should note that the typical absorption length of a synchrotron radiation photon is much greater than the escape depth of a photoelectron with the same energy, so most of the photoelectrons never leave the surface. For example, in pure $\mathrm{Cu}$ a $100-\mathrm{eV}$ photon has an absorption length $\sim 160 \AA,{ }^{24}$ whereas a $100-\mathrm{eV}$ electron has an escape depth of $\sim 7 \AA . .^{25}$ The first $100 \AA$ or so of a technical metal surface contains a lot of $\mathrm{H}, \mathrm{C}$, and $\mathrm{O}$ and is not pure $\mathrm{Cu}$ by any means, but this illustrates the idea. As a result photodesorbed atoms are expected to be initially produced and to recombine at some depth inside the near-surface oxide layer and beam tube material rather than right at the surface. After diffusing to the surface these molecules may remain physisorbed to the cold beam tube rather than thermally desorbing, as they would at room temperature. This is the reason for introducing the two desorption coefficients $\eta_{1}$ and $\eta_{2}$ in Eqs. (13) and (14) for tightly bound $\mathrm{H}, \mathrm{C}$, and $\mathrm{O}$ not previously desorbed. In the actual experiments that will be described in Section 3, it turns out that the sum of $\eta_{1}$ and $\eta_{2}$ is measured.

Throughout this paper we simply take the photodesorption coefficients as objects either to be measured by experiments or to be given by experiments and used for predicting what will happen in an accelerator. It is possible to develop a diffusion model of the photodesorption coefficient and use the experimental data to fit the parameters of the model. ${ }^{26,27}$ Some recent work along these lines, fitting the photodesorption data obtained in SSC experiments, is described in Reference 28. 


\subsection{The Solutions}

We now turn to the solutions of the equations given in the previous section. Solutions are given for a simple beam tube and for a beam tube with a liner. We are particularly interested in the effects of the finite length of the beam tube. This may not be an obvious consideration since the circumferences of the storage rings we are considering are so large. However, it is important for understanding the end effects of photodesorption experiments and also for establishing the shortest length of beam tube that can be effectively pumped from the ends. As a matter of terminology we will refer to a long beam tube as one where axial diffusion may be neglectedi.e., the density in the beam tube is independent of the end boundary conditions-and to a short beam tube as one where axial diffusion must be included. In a long cold beam tube without a liner, the surface of the tube is effectively the only pump and the surface density of physisorbed molecules will build up continuously. In a short tube the surface density will reach a steady state owing to axial diffusion or pumping out the ends. Initially beam tubes of any length obey the long-tube approximation, but if one waits long enough all tubes eventually obey the short-tube approximation. The transition time from long to short approximations is the axial diffusion time. The axial diffusion time due to thermal desorption at LHe temperatures is very long, but shortens considerably in the presence of synchrotron radiation due to repeated photodesorption ("recycling") of the physisorbed molecules, described by the $\eta$ ' desorption coefficient in Eq. (13). These comments will be verified in detail with the analysis below.

\subsubsection{Short Beam Tube Without a Liner} is

The quasi-static solution to Eq. (13) for density $n(z)$ with $V \frac{\partial n}{\partial t} \approx 0, C=0$, and $A \frac{\partial s}{\partial t} \approx 0$

$$
n(z)=\frac{1}{2} \frac{\left(\eta_{1}+\eta_{2}\right) \dot{\Gamma}}{A_{c} D}\left[\left(\frac{L}{2}\right)^{2}-z^{2}\right]+n\left( \pm \frac{L}{2}\right)
$$

where $n\left( \pm \frac{L}{2}\right)$ is the density at the ends $\pm L / 2$ of the tube. We have assumed that the desorption coefficients $\eta_{1}$ and $\eta_{2}$ are axially uniform. At the center of the tube, the density at the ends simply adds to the density due to photodesorption. A model of beam tube density at the ends is given in Section 2.2.5. Given the density $n(z)$, the surface density $s(z)$ is obtained by solving the following implicit equation for $s$ :

$$
\eta^{\prime}(s)+\frac{A_{w} s}{\dot{\Gamma} \tau_{w}^{t}(s)}=\eta_{2}+\frac{\sigma_{w} \dot{S}_{w}}{\dot{\Gamma}} n(z) .
$$

If $n_{e} \ll n(z)$ and $\eta_{2} \ll \eta^{\prime}(s)$, then

$$
\eta^{\prime}(s)=\frac{\sigma_{w} S_{w}}{\dot{\Gamma}} n(z) .
$$

If we make the further substitution $\eta^{\prime}=\eta_{0}^{\prime}\left(\frac{s}{s_{m}}\right)$, the surface density is given explicitly by

$$
s(z)=\left(\frac{\sigma_{w} S_{w}}{\eta_{0}^{\prime} \dot{\Gamma}} n(z)\right) s_{m} .
$$


We note that the diffusion out the ends of the tube is driven by isotherm evaporation and $\eta^{\prime}$ desorption. If diffusion by $\eta^{\prime}$ is negligible, then isotherm evaporation alone drives the diffusion, and $s$ is given implicitly by

$$
\frac{A_{w} s}{\dot{\Gamma} \tau_{w}^{t}(s)}=\eta_{2}+\frac{\sigma_{w} S_{w}}{\dot{\Gamma}} n(z) \text {. }
$$

The form of the solution given by Eq. (18) is exactly what would pertain to the density in a warm tube photodesorption experiment, which is not too surprising since the surface of the beam tube is in a quasi-steady state, $A \frac{\partial s}{\partial t} \approx 0$, and therefore has zero net pumping speed. This suggests that one way to determine $\eta_{1}+\eta_{2}$ is to measure the density difference between the center and ends of a short tube:

$$
\begin{aligned}
\left(\eta_{1}+\eta_{2}\right) \dot{\Gamma} L & =\frac{8}{3} \frac{\pi a^{3} \bar{v}}{\left(\frac{L}{2}\right)}\left(n(0)-n\left( \pm \frac{L}{2}\right)\right) \\
& =4 C_{L / 2}\left(n(0)-n\left( \pm \frac{L}{2}\right)\right),
\end{aligned}
$$

where $C_{L / 2}=\frac{2}{3} \frac{\pi a^{3} \bar{v}}{\left(\frac{L}{2}\right)}$ is the conductance of a tube with length $L / 2$. The measurement of $\eta_{1}+\eta_{2}$ by Eq. (23) is tantamount to setting the generation rate of gas equal to the conductance of the tube multiplied by the drop in density from the center to the ends. Two factors of two multiply the conductance compared to the usual expression $Q=C\left(p_{1}-p_{2}\right)$, because there are two parallel lengths $L / 2$ of tube from the center to the ends and because the gas is generated uniformly along the tube rather than all at the center.

A second way to measure $\eta_{1}+\eta_{2}$ is to measure the amount of gas escaping the ends, $2 A_{c} D d n / d z( \pm L / 2)$. Adding Eqs. (13) and (14) with $V \frac{\partial n}{\partial t} \approx 0, C=0$, and $A \frac{\partial s}{\partial t} \approx 0$, and integrating over $z$ yields

$$
\begin{aligned}
\left(\eta_{1}+\eta_{2}\right) L \dot{\Gamma} & =2 A_{c} D \frac{d n}{d z}( \pm L / 2) \\
& =\frac{4}{3} \pi a^{3} \bar{v} \frac{d n}{d z}( \pm L / 2) .
\end{aligned}
$$

Measurement of the right hand side would ordinarily be done by measuring the density drop across a room-temperature aperture with known conductance. Assuming that thermal transpiration, $n \bar{v}=$ constant, holds between the end of the cold tube and the room-temperature aperture, it is not necessary to know the velocity of molecules inside the cold tube to determine $\eta_{1}+\eta_{2}$. Yet a third way to measure $\eta_{1}+\eta_{2}$ with a beam tube plus liner will be discussed in Section 2.2.4. The two methods just described correspond to the ways that room-temperature desorption coefficients are usually measured. ${ }^{4,29}$ A method of separately measuring $\eta_{1}$ and $\eta_{2}$ will be described in Section 6.1. 


\subsubsection{Long 4.2-K Beam Tube Without a Liner}

The solution to Eq. (13) for $n(z)$ with $V \frac{\partial n}{\partial t} \approx 0, C=0$, and $A \frac{\partial s}{\partial t} \neq 0$ is

$$
n(z)=\left[\frac{\eta_{1} \dot{\Gamma}}{\sigma_{w} S_{w}}+\frac{\eta^{\prime} \dot{\Gamma}}{\sigma_{w} S_{w}}+\frac{1}{\sigma_{w} S_{w}} \frac{A_{w} S}{\tau_{w}^{t}}\right] \times\left[1-\frac{\cosh \left(\frac{z}{\lambda}\right)}{\cosh \left(\frac{L}{2 \lambda}\right)}\right]+n\left( \pm \frac{L}{2}\right) \times\left[\frac{\cosh \left(\frac{z}{\lambda}\right)}{\cosh \left(\frac{L}{2 \lambda}\right)}\right],
$$

where $\lambda^{2}=\frac{A_{c} D}{\sigma_{w} S_{w}}=\frac{4}{3} \frac{a^{2}}{\sigma_{w}}$ and we neglect possible $z$-dependence of $\eta_{1}, \eta^{\prime}$, and $n_{e}$. The mean square axial distance a molecule travels before sticking to the wall is $2 \lambda^{2}=\frac{8}{3} \frac{a^{2}}{\sigma_{w}}$, and $L^{2} / \lambda^{2}$ is equal to the wall pumping speed divided by the conductance of a tube of length $L$. Evidently, as long as we are a few tube diameters from the end of the tube, $L / 2-|z| \ll \lambda$, the cosh terms are negligible and we have

$$
n=\frac{\eta_{1} \dot{\Gamma}}{\sigma_{w} S_{w}}+\frac{\eta^{\prime} \dot{\Gamma}}{\sigma_{w} S_{w}}+\frac{1}{\sigma_{w} S_{w}} \frac{A_{w} S}{\tau_{w}^{t}}
$$

which is the solution obtained with axial diffusion neglected, $A_{c} D \frac{\partial^{2} n}{\partial z^{2}} \approx 0$. Unlike the shorttube solution given by Eq. (18), the density at the center of a long tube is independent of the density at the ends. Inserting Eq. (26) into Eq. (14) we obtain for the surface density

$$
s=\frac{1}{A_{w}} \int_{0}^{\Gamma}\left(\eta_{1}+\eta_{2}\right) d \Gamma,
$$

which just says all the desorbed gas winds up on the surface.

We note the similarity between Eq. (26) for the volume density in a long tube and Eq. (19) for the surface density at the center of a short tube rewritten as an equation for the density:

$$
n(0)=-\frac{\eta_{2} \dot{\Gamma}}{\sigma_{w} S_{w}}+\frac{\eta^{\prime} \dot{\Gamma}}{\sigma_{w} S_{w}}+\frac{1}{\sigma_{w} S_{w}} \frac{A_{w} S}{\tau_{w}^{t}} .
$$

If $\eta^{\prime} \gg \eta_{1}, \eta_{2}$ then Eqs. (26) and (28) are identical. Referring ahead to the experiments, it turns out that $\eta^{\prime} \gg \eta_{1}, \eta_{2}$ is a good approximation for a cryosorbing beam tube at $\mathrm{LHe}$ temperatures except at the very earliest moments of exposure to photon flux, when the surface density of physisorbed hydrogen is extremely low (i.e., $\left.s / s_{m}<\left(\eta_{1}+\eta_{2}\right) / \eta^{\prime} 0\right)$. What this means is that measurement of the dynamic density increase at the center of a cryosorbing beam tube, long or short, will determine $\eta^{\prime}$ when exposed to synchrotron radiation. Measurement of the static density with the synchrotron radiation gated off will determine the isotherm density, $n_{e}=\frac{4}{\sigma_{w}^{t} \bar{v}_{t}} \frac{s}{\tau_{w}^{t}}$. In practice one wants to choose a tube long enough that a significant surface density $s \cong s_{m}$ accumulates before the transition from the long to short solution. In this way the 
increase in isotherm density above background will be clearly visible. If the isotherm density $n_{e}$ has previously been measured as a function of surface density $s$, then measurement of $n_{e}$ during a photodesorption experiment can serve as a measurement of $s$ and the parametric dependence of $\eta^{\prime}$ on $s$ can be determined. In any case, unlike measurement of $\eta_{1}+\eta_{2}$, it is necessary to measure the density inside a cryosorbing beam tube to determine the desorption coefficient of physisorbed gas, $\eta^{\prime}$. Measurement of the flux of photodesorbed gas leaving a beam tube exposed to synchrotron radiation can only determine the photodesorption coefficient of tightly bound gas not previously desorbed. Exceptions to these conclusions arise if the assumed quasistatic conditions are violated. For example, one could consider preloading a short tube with physisorbed $\mathrm{H}_{2}$ and measuring the flux of gas leaving the ends when it is exposed to synchrotron radiation. In this case $A_{w} \frac{\partial s}{\partial t} \neq 0$. The exponential decay time of $\mathrm{H}_{2}$ leaving the ends of the tube, $\tau=\frac{A_{w} s_{m}}{\eta_{0}^{\prime} \dot{\Gamma}}$, would serve as a measurement of $\eta^{\prime}$. The time scale for this type of experiment, however, would be very short, of the order of minutes, compared to the usual photodesorption experiment, which extends over several days.

\subsubsection{Beam Tube Length Criterion, Axial Diffusion Times}

For given beam tube dimensions and desorption coefficients one can question whether the short-tube solution (A) (Eq. (18)) or the long-tube solution (B) (Eq. (26)) applies. The answer is the solution that predicts the lowest density. At the center of the beam tube this can be determined by calculating the density ratio of the two solutions:

$$
\begin{aligned}
S & =\frac{n_{B}(0)}{n_{A}(0)} \\
& =\frac{2 \lambda^{2}}{\left(\frac{L}{2}\right)^{2}} \frac{\eta^{\prime}+\eta_{1}+\frac{1}{\dot{\Gamma}} \frac{A_{w} s}{\tau_{w}^{t}}}{\eta_{1}+\eta_{2}}\left[\frac{1}{1+\frac{n_{L / 2}}{n_{A}^{0}}}\right]
\end{aligned}
$$

where $n_{A}^{0}=\frac{1}{2} \frac{\left(\eta_{1}+\eta_{2}\right)}{A_{c} D}\left(\frac{L}{2}\right)^{2}$, and we have assumed that the tube is long compared to the mean axial distance a molecule travels before sticking, $(L / 2)^{2} \gg \lambda^{2}=(4 / 3) a^{2} / \sigma_{w}$. If $S<1$, the long-tube solution applies; if $S>1$, the short-tube solution.

We can make several physical observations from the form of $S$ in Eq. (29). For the moment ignore the density at the end and set $n_{L / 2}=0$. If $\eta^{\prime}$ and $\frac{1}{\dot{\Gamma}} \frac{A_{w} s}{\tau_{w}^{t}}$ are negligible compared to $\eta_{1}$, then $S \ll 1$ and the long-tube solution pertains. This is reasonable because if $\eta^{\prime}$ and $n_{e}$ are negligible, a desorbed, tightly bound molecule travels only an rms distance $\sqrt{2} \lambda$ before sticking, and recycling of physisorbed molecules due to photo and thermal desorption is negligible. The most interesting cases are when $\eta^{\prime}$ and/or $\frac{1}{\dot{\Gamma}} \frac{A_{w} s}{\tau_{w}^{t}}$ is large compared to $\eta_{1}$. In these cases recycling of physisorbed molecules is important and, depending on circumstances, axial diffusion or pumping out the ends of the tube may $(S>1)$ or may not $(S<1)$ be impor- 
tant. We imagine starting a photodesorption experiment with an initially low surface density of physisorbed gas and, therefore, low $\eta^{\prime}=\eta_{0}^{\prime}\left(s / s_{m}\right)$ and $n_{e}$. In these circumstances a beam tube will tend to look long, $S<1$. As the experiment proceeds, $s, \eta^{\prime}$, and $n_{e}$ will increase and eventually the short-tube region, $S>1$, will be reached. It is interesting to calculate the transition time $T_{D}$ between the solutions A (Eq. (18)) and B (Eq. (26)). For simplicity we assume $\eta_{1}+\eta_{2}$ is constant so that the surface density of physisorbed $\mathrm{H}_{2}$ at the transition time is related to $T_{D}$ by $A_{w} s=\left(\eta_{1}+\eta_{2}\right) \dot{\Gamma} T_{D}$. Substituting into Eq. (29) with $S=1$ we obtain

$$
\frac{1}{T_{D}}=\frac{2 \lambda^{2}}{\left(\frac{L}{2}\right)^{2}}\left[\frac{\eta_{0}^{\prime} \dot{\Gamma}}{A_{w} s_{m}}+\frac{1}{\tau_{w}^{t}}\right]
$$

The scaling of $T_{D}$ with $(L / \lambda)^{2}$ as well as the physical context suggests that the transition time is related to axial diffusion. This connection can be made explicit by calculating the time it takes a molecule to diffuse a distance $L / 2$ from the center of the beam tube to the ends. The rms axial step size per desorption is $\sqrt{2} \lambda$. The mean number of $\eta^{\prime}$ desorptions of a given molecule per second is $\frac{\eta^{\prime} \dot{\Gamma}}{A_{w} s}$, so we obtain for the mean time $T_{D, \eta^{\prime}}$ for a molecule to diffuse a distance $L / 2$ by $\eta^{\prime}$ desorption:

$$
\begin{aligned}
T_{D, \eta^{\prime}} & =\frac{\left(\frac{L}{2}\right)^{2}}{2 \lambda^{2}} \frac{A_{w s}}{\eta^{\prime} \dot{\Gamma}} \\
& =\frac{\left(\frac{L}{2}\right)^{2}}{2 \lambda^{2}} \frac{A_{w} s_{m}}{\eta_{0}^{\prime} \dot{\Gamma}}
\end{aligned}
$$

Similarly, the mean thermal desorption time for a given molecule is $\tau_{w}^{t}$, so we obtain for the time $T_{D, t}$ for a molecule to diffuse a distance $L / 2$ by thermal desorption:

$$
T_{D, t}=\frac{\left(\frac{L}{2}\right)^{2}}{2 \lambda^{2}} \frac{1}{\tau_{w}^{t}}
$$

Comparing to Eq. (30), the connection between the transition time and diffusion times is clear:

$$
\frac{1}{T_{D}}=\frac{1}{T_{D, \eta^{\prime}}}+\frac{1}{T_{D, e}} .
$$

The diffusion processes are parallel, so the diffusion times add reciprocally. The long-tube solution (Eq. (26)) goes over to the diffusion-dominated solution (Eq. (18)) on the time scale it takes a molecule to diffuse from the center to the ends at $\pm L / 2$. The diffusion solution evolves inward from the ends and reaches the center in a time $T_{D}$. We note that a given length tube can be both long and short depending on the time scale; in the past this has 
sometimes caused objections to the terminology. However, it seems to the author to be a useful shorthand for referring to diffusion-negligible and diffusion-dominated situations. Perhaps the terminology loses something in translation. Numerical values of the axial diffusion times and related quantities will be given in Section 4.1 after discussion of some experimental data.

From Eq. (32) the thermal diffusion time $T_{D, t}$ is simply $\frac{\left(\frac{L}{2}\right)^{2}}{2 \lambda^{2}}$ times the thermal sojourn time $\tau_{w}^{t}$. It is clear from Eq. (30) that we can generalize the sojourn time concept to include photodesorption. The mean time for photodesorption of a physisorbed molecule is $\frac{A_{w} s}{\eta^{\prime} \dot{\mathrm{T}}}$, which defines the photodesorption sojourn time $\tau_{w}^{\eta^{\prime}}$ :

$$
\begin{aligned}
\tau_{w}^{\eta^{\prime}} & =\frac{A_{w} s}{\eta^{\prime} \dot{\Gamma}} \\
& =\frac{A_{w} s_{m}}{\eta_{0}^{\prime} \dot{\Gamma}} \text { for } \eta^{\prime}=\eta_{0}^{\prime}\left(\frac{s}{s_{m}}\right),
\end{aligned}
$$

and the sojourn time $\tau_{w}$ due to the combined effects of thermal desorption and photodesorption is

$$
\frac{1}{\tau_{w}}=\frac{1}{\tau_{w}^{t}}+\frac{1}{\tau_{w}^{\eta^{\prime}}}
$$

Introducing the photodesorption sojourn time $\tau_{w}^{\eta^{\prime}}$ is merely an alternative way of expressing the photodesorption of physisorbed molecules; we could have started this way in Eq. (13) by replacing $\eta^{\prime} \dot{\Gamma}$ by $\frac{A_{w} s}{\tau_{w}^{\eta^{\prime}}}$. Numerical estimates of the sojourn times defined here are given in Section 4.1.

We now return to the effect of density at the end of the tube on $S$, given by Eq. (29). Since increasing $n_{L / 2}$ decreases $S$, increasing the density at the ends tends to drive the solution toward the long-tube case. The reason is that diffusion into the tube from the ends partially compensates what diffuses out. Since $n_{A}(0)=n_{A}^{0}+n_{L / 2}$, the density-dependent term in Eq. (29) can be rewritten as

$$
\frac{1}{1+\frac{n_{L / 2}}{n_{A}^{0}}}=1-\frac{n_{L / 2}}{n_{A}(0)} \text {. }
$$

In an extreme case with $n_{L / 2}=n_{A}(0)$, diffusion into the tube exactly balances diffusion out, $S=0$, and the beam tube looks infinitely long. Short-tube solutions exist only for $n_{L / 2}<n_{A}(0)$, whereas long-tube solutions can have $n_{L / 2}<n_{A}(0)$ or $n_{L / 2}>n_{A}(0)$. If $n_{L / 2}>n_{A}(0)$, the surface density cannot reach a steady state and the surface of the beam tube is forced to continue pumping. To some extent $n_{L / 2}$ can be controlled by varying the pumping speed at the ends of the beam tube. Reaching $n_{L / 2}>n_{A}(0)$ requires a source of gas at the ends in addition to photodesorbed gas from the beam tube. These techniques can be useful for extending the range of long-tube behavior and measuring $\eta^{\prime}$ at high values of $s / s_{m}$ that otherwise would be limited by diffusion out of the beam tube. 


\subsubsection{Beam Tube With a Liner}

The case of a beam tube with a liner is only interesting when $C n \gg A_{c} D \partial^{2} n / \partial z^{2}$. In that case, and with the quasi-static approximations $V \frac{\partial n}{\partial t} \approx 0$ and $A \frac{\partial s}{\partial t} \approx 0$, the solution for the density is

$$
n=\frac{\left(\eta_{1}+\eta_{2}\right) \dot{\Gamma}}{C}
$$

Equation (37) is remarkable in its simplicity compared to Eq. (26). It depends only on the desorption coefficient $\eta=\eta_{1}+\eta_{2}$ to specify a conductance $C$ that will meet the beam lifetime criterion. Equation (37) is valid for any temperature liner, independently of whether there is cryosorption on the liner surface. The conductance $C$ is related to the holes in the liner by $C=p N_{h} A_{h} \bar{v} / 4$, where $p$ is the transmission probability, $N_{h}$ the number of holes per unit axial length, $A_{h}$ the area per hole, and $\bar{\nu}$ the mean molecular speed. Although the mean speed $\bar{\nu}$ may be higher than that corresponding to equilibrium with the liner temperature, a conservative estimate of the conductance may be made by assuming the molecules and liner are in thermal equilibrium. Equation (37) suggests that an effective way to measure the desorption coefficient $\eta_{1}+\eta_{2}$ of tightly bound molecules is to measure the density of molecules inside a beam tube with a liner. This method has some advantages compared to the methods suggested by Eq. (18) for the beam tube without a liner. The density needs only to be measured at one axial location. One need not worry about generation of gas in the warm-end regions due to imperfect collimation or about pumping by the synchrotron radiation beamline. Measurement of $\eta_{1}+\eta_{2}$ is precisely what is needed to decide the issue of liner or no liner when vacuum quality is the deciding issue, since $\eta_{1}+\eta_{2}$ integrated over the photon flux $\Gamma$ gives the amount of desorbed $\mathrm{H}_{2}$ and, therefore, the frequency of beam tube warm-ups required in a beam tube without a liner. It is paradoxical that the best way to decide this issue is an experiment with a liner configuration.

If the liner is cold enough to be cryosorbing, the solution for the surface density is the same as Eqs. (19)-(21) for the short-tube solution of the cryosorbing beam tube without a liner. In fact, as we saw with Eq. (28), Eq. (19) can be rewritten to look like the long-tube solution (Eq. (26)) but with $\eta_{1}$ replaced by $-\eta_{2}$. Because of this one might think that the liner configuration could be useful to measure $\eta^{\prime}$ as well as $\eta_{1}+\eta_{2}$. In practice this turns out not to be the case because the liner conductance term has been chosen to be large compared to axial diffusion. In this case the surface density of physisorbed $\mathrm{H}_{2}$ only reaches a low value before it saturates, and the isotherm density is too small to be observable above background. In fact, the usual situation for a liner experiment is that $\eta_{2} \ll \eta^{\prime}, n_{e} \ll n$ so that $\eta_{1}+\eta_{2}$ and $\eta^{\prime}$ are related by

$$
\eta^{\prime}=\frac{\sigma_{w} S_{w}}{C}\left(\eta_{1}+\eta_{2}\right)
$$

and from this $s$ may be estimated if $\eta_{0}^{\prime}$ is known.

\subsubsection{Model of Beam Tube Density at the Ends}

In several places we have dealt with the boundary condition at the end of a beam tube by simply specifying the density $n_{L / 2}$. In practice this density is determined by a number of factors: 
the gas flux into or out of the tube ends, the effective pumping speed at the ends of the tube, and the magnitude of gas source at the tube ends. In this section we will give some formulas for $n_{L / 2}$ in terms of these factors. Usually the effective pumping speed is determined by roomtemperature vacuum components that are connected to the cold tube end by thin-walled bellows while the photodesorbed gas is coming out of the tube with a different temperature. We will first consider the artificial case where all the vacuum components are at the same temperature as the gas leaving the beam tube $\left(T_{1}\right)$, and then consider the changes when the components are at room temperature $\left(T_{2}\right)$.

Suppose there is a source of gas $Q_{\text {ext }}$ external to the beam tube and an effective pumping speed $S_{\text {ext }}$ at the tube ends $\pm L / 2$. Continuity of mass flow then gives $Q_{\text {ext }}+Q_{D}=S_{\text {ext }} n_{L / 2}$, where $Q_{D}=-\left.A_{c} D \frac{d n}{d z}\right|_{ \pm L / 2}$ is the gas diffusing into or out of the tube at $\pm L / 2$. For the shorttube solution (Eq. (18)), $Q_{D}=\left(\eta_{1}+\eta_{2}\right) \dot{\Gamma} \frac{L}{2}$. Inserting this into the continuity equation gives the following result for $\frac{n_{L / 2}}{n_{A}^{0}}$ in Eq. (29):

$$
\frac{n_{L / 2}}{n_{A}^{0}}=\frac{2 C_{L / 2}}{S_{\mathrm{ext}}}\left(1+\frac{Q_{\mathrm{ext}}}{\left(\eta_{1}+\eta_{2}\right) \dot{\Gamma} \frac{L}{2}}\right)
$$

where the external pumping speed is normalized to the beam-tube conductance $C_{L / 2}$, and the external gas source is normalized to the photodesorbed gas in half the length of the beam tube.

In Eq. (39) we have supposed that the external pumping and gas source are right at the tube ends and at the temperature $T_{1}$ of gas leaving the tube. Now suppose a more realistic case, with the gas source and external pumping speed at room temperature and separated from the cold end of the beam tube by a room-temperature tube with conductance $C_{\text {ext }}^{\prime}$. The primed conductance $\left(C_{\text {ext }}^{\prime}\right)$ and pumping speed $\left(S_{\text {ext }}^{\prime}\right)$ are evaluated at room temperature $T_{2}$, unprimed at the cold temperature $T_{1}$ of gas leaving the beam tube. The room-temperature tube is connected to the cold end of the beam tube with a short bellows whose impedance we neglect. Applying continuity at both ends of $C_{\text {ext }}^{\prime}$ we obtain $C_{\text {ext }}^{\prime}\left(n_{2}-n_{1}\right)+Q_{\text {ext }}^{\prime}=S_{\text {ext }}^{\prime} n_{1}$, $\left(\eta_{1}+\eta_{2}\right) \dot{\Gamma} \frac{L}{2}=C_{\text {ext }}^{\prime}\left(n_{2}-n_{1}\right)$, and $n_{2} \sqrt{T_{2}}=n_{L / 2} \sqrt{T_{1}}$, where $n_{1}$ and $n_{2}$ are the room-temperature gas densities at the opposite ends of $C_{\text {ext }}^{\prime}$, and $n_{L / 2}$ is the density of gas at the end of the beam tube at temperature $T_{1}$. Note that if $T_{1} \neq T_{2}, n_{2} \neq n_{L / 2}$ and there is a step in density at $L / 2$. Using these equations to solve for $\frac{n_{L / 2}}{n_{A}^{0}}$, Eq. (39) is replaced by

$$
\frac{n_{L / 2}}{n_{A}^{0}}=\frac{2 C_{L / 2}}{\sqrt{\frac{T_{1}}{T_{2}}} C_{\text {ext }}^{\prime}}+\frac{2 C_{L / 2}}{\sqrt{\frac{T_{1}}{T_{2}}} S_{\text {ext }}^{\prime}}\left(1+\frac{Q_{\text {ext }}^{\prime}}{\left(\eta_{1}+\eta_{2}\right) \dot{\Gamma}^{\frac{L}{2}}}\right) .
$$

From the form of Eq. (40) we see that we can drop the primes and the temperature factors if pumping speeds and conductances at $T_{2}$ are evaluated as though they were at temperature $T_{1}$. 


\subsection{Ion Desorption}

So far we have included only gas desorption by synchrotron radiation. Ionization of beam tube gas by the circulating proton beam followed by acceleration of the low-energy ions into the beam tube wall by the beam space-charge fields can cause ion-induced desorption. Because the ion desorption rate depends linearly on the gas density, the process is regenerative and can lead to the well-known ion pressure bump instability. ${ }^{30}$ Furthermore, the ion desorption coefficient for physisorbed gas is known to be very high. ${ }^{31}$ So in the present context of a cold cryosorbing beam tube exposed to synchrotron radiation, it is possible that an initially stable situation may become unstable due to the accumulation of physisorbed gas by the combination of synchrotron radiation and ion bombardment desorption of tightly bound molecules and atoms. To analyze the situation we add the appropriate ion desorption terms to Eqs. (13) and (14):

$$
\begin{gathered}
V \frac{\partial n}{\partial t}=\eta_{i} \frac{I \sigma_{p i}}{e} n+\eta_{i}^{\prime} \frac{I \sigma_{p i}}{e} n+\eta_{1} \dot{\Gamma}+\eta^{\prime} \dot{\Gamma}-\sigma_{w} S_{w} n+\frac{A_{w} s}{\tau_{w}^{t}}-C * n+A_{c} D \frac{\partial^{2} n}{\partial z^{2}} \\
A_{w} \frac{\partial s}{\partial t}=\eta_{2} \dot{\Gamma}+\sigma_{w} S_{w} n-\frac{A_{w} s}{\tau_{w}^{t}}-\eta^{\prime} \dot{\Gamma}-\eta_{i}^{\prime} \frac{I \sigma_{p i}}{e} n
\end{gathered}
$$

where $I$ is the proton beam current, $\sigma_{p i}$ is the proton ionization cross section for $\mathrm{H}_{2}, \eta_{i}$ is the net ion desorption coefficient of tightly bound hydrogen $\left(\eta_{i} \geq-1\right)$, and $\eta_{i}^{\prime}$ is the ion desorption coefficient of physisorbed $\mathrm{H}_{2}$. Molecules that are pumped by proton ionization are assumed not to contribute to the physisorbed molecules. If all of them did, for example, then there would be an additional term on the right hand side of Eq. (42), $\left(=\frac{I \sigma_{p i}}{e} n\right)$. Following our treatment of the photodesorption of physisorbed gas, we define a linear ion desorption coefficient at low surface coverage: $\eta_{i}^{\prime}=\eta_{i 0}^{\prime}\left(\frac{s}{s_{m}}\right)$. The data of Hilleret and Calder ${ }^{31}$ show this linear behavior up to $s \sim 10^{16} / \mathrm{cm}^{2}$ for the desorption of physisorbed $\mathrm{H}_{2}$ on a $3.2-\mathrm{K}$ stainless steel surface bombarded by $0.5-10 \mathrm{keV} \mathrm{H}_{2}{ }^{+}$. We now turn to the solutions of these equations in the same order given previously without ion desorption: short beam tube without a liner, long $4.2-\mathrm{K}$ beam tube without a liner, and beam tube with a liner.

Short beam tube without a liner

The solution to Eqs. (41) and (42) with $V \frac{\partial n}{\partial t} \approx 0, C=0$, and $A \frac{\partial s}{\partial t} \approx 0$ is

$$
\begin{gathered}
n=\frac{\left(\eta_{1}+\eta_{2}\right) \dot{\Gamma}}{\eta_{i} \frac{I \sigma_{p i}}{e}}\left(1-\frac{\cos \left(\frac{z}{\lambda_{\eta_{i}}}\right)}{\left.\cos \left(\frac{L}{2 \lambda_{\eta_{i}}}\right)\right)+\frac{\cos \left(\frac{z}{\lambda_{n_{i}}}\right)}{\cos \left(\frac{L}{2 \lambda_{\eta_{i}}}\right)} n\left( \pm \frac{L}{2}\right)}\right. \\
s=\frac{\eta_{2} \dot{\Gamma}+\sigma_{w} S_{w} n}{\eta_{0}^{\prime} \dot{\Gamma}+\eta_{i 0}^{\prime}\left(\frac{I \sigma_{p i}}{e} n\right)+\frac{A_{w}}{\tau_{w}^{t}}} s_{m},
\end{gathered}
$$


where $\frac{1}{\lambda_{\eta_{i}}^{2}}=\frac{\eta_{i}}{A_{c} D} \times \frac{I \sigma_{p i}}{e}$. We have assumed that the ion desorption coefficient $\eta_{i}$ is positive. If $\eta_{i}$ is negative then the cosines are replaced by hyperbolic cosines, the solution goes over to the form of Eq. (25), and it is stable. If $\eta_{i}$ is positive, then from Eq. (43) we have the geometric stability condition ${ }^{30} \frac{L}{2 \lambda_{\eta_{i}}}<\frac{\pi}{2}$, or

$$
\eta_{i} I<\frac{\pi^{2}}{4} \frac{e}{\sigma_{p i}} \times\left(\frac{2}{L}\right)^{2} \times \frac{2}{3} \pi a^{3} \bar{v} .
$$

This is the same as the familiar result for ion desorption stability. If it is violated there is no quasi-static solution $V \frac{\partial n}{\partial t} \approx 0$, and an initially finite beam tube density will grow exponentially with time.

\section{Long 4.2-K beam tube without a liner}

The solution to Eqs. (41) and (42) with $V \frac{\partial n}{\partial t} \approx 0, C=0$, and $A \frac{\partial s}{\partial t} \neq 0$ is

$$
\begin{gathered}
n=\frac{\eta_{1} \dot{\Gamma}+\eta^{\prime} \dot{\Gamma}+\frac{A_{w} s}{\tau_{w}^{t}}}{\sigma_{w} S_{w}-\eta_{i} \frac{I \sigma_{p i}}{e}-\eta_{i}^{\prime} \frac{I \sigma_{p i}}{e}} \\
s=\frac{1}{A_{w}} \int_{0}^{\Gamma}\left[\eta_{1}+\eta_{2}+\left(\frac{I \sigma_{p i}}{e \dot{\Gamma}} n\right) \eta_{i}\right] d \Gamma,
\end{gathered}
$$

where we have assumed that $\lambda^{2}=\frac{A_{c} D}{\sigma_{w} S_{w}}=\frac{4}{3} \frac{a^{2}}{\sigma_{w}} \ll L^{2}$, and we are at least a few diameters from the end of the tube. If the denominator in Eq. (46) vanishes or is negative, there is no net pumping by the wall and the vacuum is unstable. Stability then requires that

$$
\left(\eta_{i}+\eta_{i}^{\prime}\right) I<\frac{e}{\sigma_{p i}} \sigma_{w} S_{w}
$$

Compared to Eq. (45), pumping by axial conductance has been replaced by the potentially much larger cryopumping on the beam tube wall. However, the desorption coefficient of tightly bound hydrogen has been replaced by the sum of ion desorption coefficients for tightly bound and physisorbed hydrogen, which is also potentially much larger. Hilleret and Calder have reported $\eta_{i}^{\prime} \approx 700 \mathrm{H}_{2}$ /ion for $500 \mathrm{eV} \mathrm{H}{ }_{2}{ }^{+}$incident on a surface with $10^{15} \mathrm{H}_{2} / \mathrm{cm}^{2}$ of physisorbed $\mathrm{H}_{2}$ at $3.2 \mathrm{~K}^{31}$ Clearly ion desorption of physisorbed $\mathrm{H}_{2}$ could be a serious threat to the stability of a cryosorbing beam tube. 
Beam tube with a liner

For the case of a beam tube with a liner chosen so that $C n \gg A_{c} D \partial^{2} n / \partial z^{2}$, and with the quasi-static approximations $V \frac{\partial n}{\partial t} \approx 0$ and $A \frac{\partial s}{\partial t} \approx 0$, the solution to Eqs. (41) and (42) is

$$
\begin{gathered}
n=\frac{\left(\eta_{1}+\eta_{2}\right) \dot{\Gamma}}{C-\eta_{i} \frac{I \sigma_{p i}}{e}} \\
s=\frac{\eta_{2} \dot{\Gamma}+\sigma_{w} S_{w} n}{\eta_{0}^{\prime} \dot{\Gamma}+\eta_{i 0}^{\prime}\left(\frac{I \sigma_{p i}}{e} n\right)+\frac{A_{w} s_{m}}{\tau_{w}^{t}}} s_{m} \\
\approx \frac{\sigma_{w} S_{w} n}{\eta_{0}^{\prime} \dot{\Gamma}+\eta_{i 0}^{\prime}\left(\frac{I \sigma_{p i}}{e} n\right)} .
\end{gathered}
$$

From the denominator of Eq. (49) stability now requires that pumping through the conductance of the liner holes exceed the ion desorption rate of tightly bound hydrogen:

$$
\eta_{i} I<\frac{e}{\sigma_{p i}} C .
$$

Although the liner pumping speed $C$ is perhaps a few percent of the pumping speed of the cold beam tube surface $\sigma_{w} S_{w}$ in Eq. (48), the stability condition is improved by the absence of $\eta_{i}^{\prime}$. Even if the liner is cold enough that there is a finite density of physisorbed $\mathrm{H}_{2}$ on its surface given by Eq. (50), the ion desorption coefficient of physisorbed $\mathrm{H}_{2}$ does not appear in the denominator of Eq. (49) and hence does not enter into the stability condition. The ion desorption of physisorbed $\mathrm{H}_{2}$ is balanced by pumping through the holes in the liner. One can see from Eq. (50) that the surface density of $\mathrm{H}_{2}$ physisorbed on the liner is lower in the presence of ion desorption than without it. In effect ion desorption simply increases the rate at which $\mathrm{H}_{2}$ is moved to the region behind the liner, and together with photodesorption it prevents the build-up of thick adsorbed layers on the liner. From the viewpoint of vacuum stability of the liner there does not appear to be any particular advantage to operating at a temperature high enough to avoid physisorbed $\mathrm{H}_{2}(\geq 20 \mathrm{~K})$.

\section{Beam Tube Photodesorption Experiments}

\subsection{Room-Temperature Experiments}

Since the experiments of Grobner et al. reported in Reference 4 there have been many measurements of room-temperature photodesorption coefficients on a variety of materials. A representative but not exhaustive collection of results is contained in References 4, 18, 19, 20, 22,29 and 32. Within the last couple of years an investigation of photodesorption from various methods of manufacturing copper was carried out at the Brookhaven National Laboratory 
National Synchrotron Light Source (BNL NSLS) UV ring. ${ }^{20}$ These experiments were motivated by the SSC. Beam tube samples ( $1 \mathrm{~m}$ long, $32 \mathrm{~mm}$ ID) were exposed to synchrotron radiation with critical energy $490 \mathrm{eV}$, angle of incidence $12 \mathrm{mrad}$, and total integrated flux $\sim 10^{23}$ photons $/ \mathrm{m}$. Samples of electrodeposited $\mathrm{Cu}$ from two manufacturers with different plating procedures (Silvex and Fluhmann) and high-purity bulk copper (Hitachi 10100) were investigated. In addition, one sample of aluminum was investigated (A7N01/4N). All samples were cleaned with the identical procedure prior to installation in the vacuum system (degrease in ultrasonic cleaner, water rinse, alcohol rinse, air dry). The standard procedure after cleaning was to install the sample beam tube in the vacuum system, pump for a few days until a base pressure $\sim 10^{-8}$ Torr was reached, and then begin the photodesorption experiments (samples $1,3,4,5,7,8$, and 9 in Table 4). Variations of this procedure were carried out on two of the Silvex samples: $24 \mathrm{~h}$, $350^{\circ} \mathrm{C}$ vacuum bake (sample 2); and $5 \mathrm{~h}, 700^{\circ} \mathrm{C}$ thermal pretreatment (sample 6). The thermal pretreatment was carried out in an apparatus at Virginia Polytechnic Institute (VPI) ${ }^{33}$ The $700^{\circ} \mathrm{C}$ thermal cycle in a flowing atmosphere of $10 \% \mathrm{H}_{2}+90 \%$ He was followed by passivation with $\sim 200-300 \AA$ of $A u$ and then exposed to atmosphere for shipment to BNL. The thermal pretreatment was observed to significantly increase the grain size and decrease the $\mathrm{Cu}$ porosity. One of the Silvex samples was immersed in a 1.1-kG vertical magnetic field, and another was coated with $2 \mu \mathrm{m}$ of Au. Samples 1-3 from Silvex were manufactured in the period 1988-90, and samples 4-6 in 1992. Photodesorption of $\mathrm{H}_{2}, \mathrm{CH}_{4}, \mathrm{CO}$, and $\mathrm{CO}_{2}$ were recorded as a function of photon exposure. In Table 4 we simply give as figures of merit the magnitudes of the $\mathrm{H}_{2}$ and $\mathrm{CO}$ photodesorption coefficients and the total amounts of desorbed gas at the end of exposure to $10^{23}$ photons $/ \mathrm{m}$, and refer to Reference 20 for additional information.

Before getting into the details we simply note that there are not large sample-to-sample variations in Table 4. Comparing sample 1 , with the largest photodesorption coefficients, to sample 6 , with the smallest photodesorption coefficients, the ratio of $\mathrm{H}_{2}$ photodesorption coefficients is 2.5 and the ratio of desorbed $\mathrm{H}_{2}$ is 3.4. Taking the $\mathrm{H}_{2}$ monolayer surface density to be $3 \times 10^{15} / \mathrm{cm}^{2}, 17$ monolayers have been desorbed from sample 1 , and 5 monolayers from sample 6 , in what amounts to half an operational year of photon flux at the SSC design intensity.

Now we comment on a few of the details in Table 4. Comparing samples 1 and 5, about 1.5 times more $\mathrm{H}_{2}$ was desorbed from the earlier production sample. than from the later, suggesting that continued exposure to atmosphere increases the amount of desorbable material. The effect is smaller for $\mathrm{CO}$. Comparing samples 1 and 2 , an in situ $350^{\circ} \mathrm{C}$ vacuum bake reduced the desorbed $\mathrm{H}_{2}$ by a factor of 3 . The $700^{\circ} \mathrm{C}$ thermal pretreatment followed by an $\mathrm{Au}$ flash obtained a slightly better ratio, 3.4. Comparing samples 4 and 5 , an Au layer without thermal pretreatment had no significant effect on the magnitude of photodesorption. Comparing samples 5 and 7 , the Silvex and Fluhmann electrodeposited $\mathrm{Cu}$ desorbed about the same amount of $\mathrm{H}_{2}$, and the desorbed $\mathrm{CO}$ was 1.7 times less for the Fluhmann sample. Comparing samples 5 and 7 with sample 8 , the amount of desorbed $\mathrm{H}_{2}$ and $\mathrm{CO}$ from the high-purity bulk $\mathrm{Cu}$ falls in the range of the electrodeposited $\mathrm{Cu}$ results. At the beginning of this undertaking, the intent was to test several other methods of $\mathrm{Cu}$ production, including chemical vapor and plasma vapor deposition (CVD and PVD). Cancellation of the SSC prevented completion of this program. However, the bulk $\mathrm{Cu}$ result obtained here suggests that there is little to be gained in the reduction of photodesorbable material by different preparation methods. Rather it seems 
that exposure to atmosphere and cleaning solutions render the photodesorption characteristics to be fairly similar and independent of the method of production. If any gain is to be obtained in the reduction of photodesorbable material, it seems that it will have to occur by some type of surface treatment following production. Finally, we see that electrodeposited $\mathrm{Cu}$ (samples 5 and 7), high-purity bulk $\mathrm{Cu}$ (sample 8), and aluminum (sample 9) have remarkably similar photodesorption coefficients.

Table 4. $\mathrm{H}_{2}$ and $\mathrm{CO}$ room-temperature photodesorption coefficients and amounts of desorbed gas after an integrated photon flux $10^{23}$ photons $/ \mathrm{m}^{20}$

\begin{tabular}{|l|c|c|c|c|}
\hline Sample & $\eta\left(\mathrm{H}_{2}\right)$ & $\eta(\mathrm{CO})$ & $\mathrm{H}_{2} / \mathrm{m}$ & $\mathrm{CO} / \mathrm{m}$ \\
\hline 1. Elec. Cu (S, 1988-1990) & $1.7 \times 10^{-4}$ & $4.4 \times 10^{-5}$ & $5.1 \times 10^{19}$ & $1.3 \times 10^{19}$ \\
\hline $\begin{array}{l}\text { 2. Elec. Cu (S, 1988-1990) with } \\
\text { 350 } \mathrm{C} \text { bake }\end{array}$ & $9.3 \times 10^{-5}$ & $1.4 \times 10^{-5}$ & $1.7 \times 10^{19}$ & $2.8 \times 10^{18}$ \\
\hline 3. Elec. Cu (S, 1988-1990) with B & $1.4 \times 10^{-4}$ & $3.0 \times 10^{-5}$ & $3.3 \times 10^{19}$ & $8.1 \times 10^{18}$ \\
\hline 4. Elec. Cu (S, 1992) with Au layer & $1.5 \times 10^{-4}$ & $1.7 \times 10^{-5}$ & $3.4 \times 10^{19}$ & $4.5 \times 10^{18}$ \\
\hline 5. Elec. Cu (S, 1992) & $1.0 \times 10^{-4}$ & $2.0 \times 10^{-5}$ & $3.5 \times 10^{19}$ & $1.0 \times 10^{19}$ \\
\hline 6. Elec. Cu (S, 1992) with thermal \\
pre-treatment & $6.8 \times 10^{-5}$ & $1.4 \times 10^{-5}$ & $1.5 \times 10^{19}$ & $2.9 \times 10^{18}$ \\
\hline 7. Elec. Cu (F, 1992) & $1.0 \times 10^{-4}$ & $2.1 \times 10^{-5}$ & $3.1 \times 10^{19}$ & $6.7 \times 10^{18}$ \\
\hline 8. High-purity bulk Cu (Hitachi 10100) & $9.2 \times 10^{-5}$ & $2.0 \times 10^{-5}$ & $2.4 \times 10^{19}$ & $7.4 \times 10^{18}$ \\
\hline 9. Al, A7N01/4N & $1.3 \times 10^{-4}$ & $2.8 \times 10^{-5}$ & $2.8 \times 10^{19}$ & $5.8 \times 10^{18}$ \\
\hline S = Silvex, F = Fluhmann & & & & \\
\hline
\end{tabular}


One experiment (sample 3 ) in Table 4 was carried out with a magnetic field strong enough to confine photoelectrons leaving the surface to within a small distance of their point of emission. The primary synchrotron radiation photons with $E=E_{1 / 2}$ illuminate a strip height with FWHM $=16 \mathrm{~mm}$, only $\sim 16 \%$ of the beam-tube circumference. If secondary electrons leaving the directly illuminated strip are responsible for a large fraction of the desorbed gas, then one would expect much faster clean-up with the applied magnetic field, which reduces their effective desorption area to $\sim 16 \%$ of the tube circumference. In fact, comparing samples 1 and 3 , the effects of magnetic field on the magnitudes of desorption coefficients and amounts of desorbed gas at $10^{23}$ photons $/ \mathrm{m}$ are not large and are arguably less than what could be expected from sampleto-sample variation. Switching the field momentarily off during the magnetic field experiment resulted in a 10-20\% increase in the magnitude of desorption coefficients. Similar results have been obtained in warm-tube experiments at the Budker Institute of Nuclear Physics (BINP). ${ }^{29}$ The conclusion of this experiment is that photodesorption experiments without magnetic field are adequate for simulating accelerator conditions with magnetic field. A corollary is that the photoelectrons leaving the directly illuminated strip are not responsible for the bulk of the desorbed gas.

Following the experiment reported as sample 2 in Table 4, the tube was rotated $140^{\circ}$ under vacuum and again exposed to photons. The magnitudes of initial desorption coefficients after rotation indicated a conditioning equivalent to approximately $25 \%$ of the integrated flux in the original orientation.

\subsection{Cryogenic Experiments}

\subsubsection{The CDG Experiments}

The first photodesorption experiments at LHe temperature were done during the Central Design Group (CDG) era (1984-89) of the SSC by the authors of References 16 and 17. Figure 4(a) shows a schematic of the apparatus of Reference 16. A 4.1-m length of 32-mm-ID beam tube in an LHe cryostat was exposed to synchrotron radiation from the BNL NSLS UV ring, with critical energy 251 and $478 \mathrm{eV}$ and angle of incidence $9.3 \mathrm{mrad}$. The photon beam could be modulated by a beam chopper that produced $\sim 3.5$-ms-wide pulses. An interesting arrangement of residual gas analyzer (RGA) detectors was used in this experiment; a schematic is shown in Figure 4(b). Each RGA is arranged in a molecular beam detector fashion; the ionizer intercepts a stream of molecules leaving a hole in the beam tube and measures the density of the stream in a single pass. This type of detector requires close coupling to the beam tube in order to get adequate sensitivity. The RGAs could be moved out of the molecular streams to correct the data for the background density of molecules that have equilibrated with the walls of the ionizer. The ratio of molecular density in the beam tube to the density in the stream passing through the RGA depends only on geometry and is independent of the molecular velocity and gas species. As a consequence, this ratio can be conveniently measured with room-temperature gas. The authors of Reference 16 did this for $\mathrm{H}_{2}$ (154), $\mathrm{He}(103), \mathrm{N}_{2}$ (93), and $\mathrm{Kr}$ (72), and reported the values given in parentheses. Monte Carlo calculations gave a value of 78 . The density ratio shouldn't depend on mass; the factor-of-two higher value for $\mathrm{H}_{2}$ than for $\mathrm{Kr}$ and the Monte Carlo calculation were not considered to be a significant disagreement outside the 
bounds of measurement accuracy. To be conservative the authors of Reference 16 used a density ratio of 150 to infer beam-tube densities from the RGA densities. Although density is measured, the authors of Reference 16 consistently report beam-tube pressure in units the same density would have at room temperature.

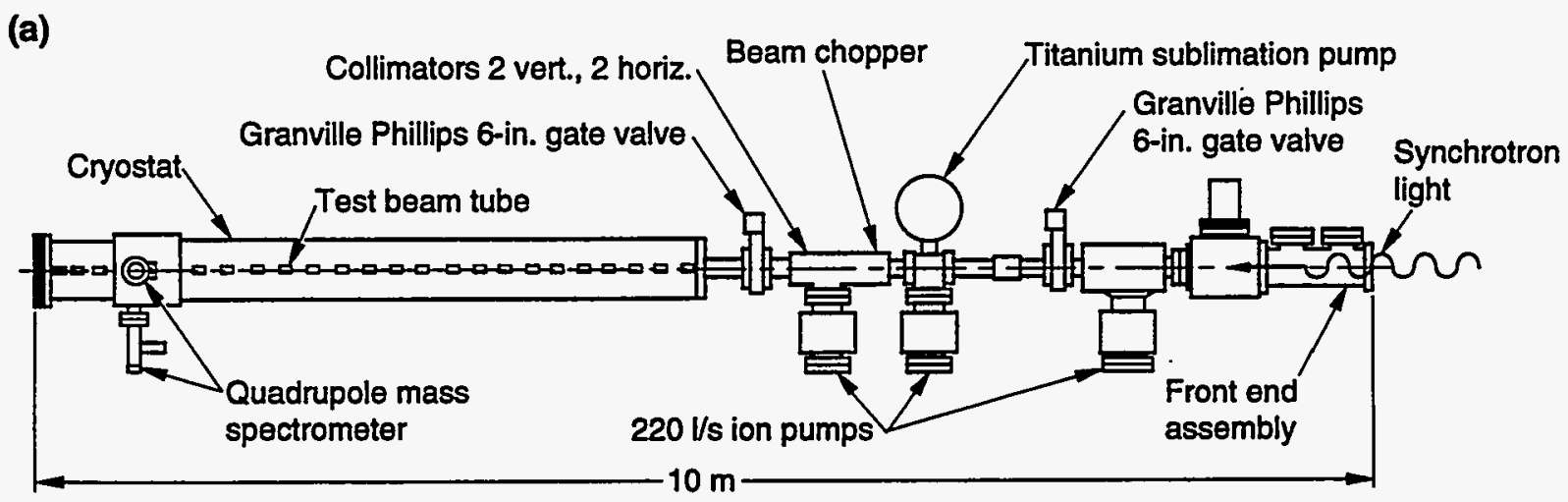

(b)

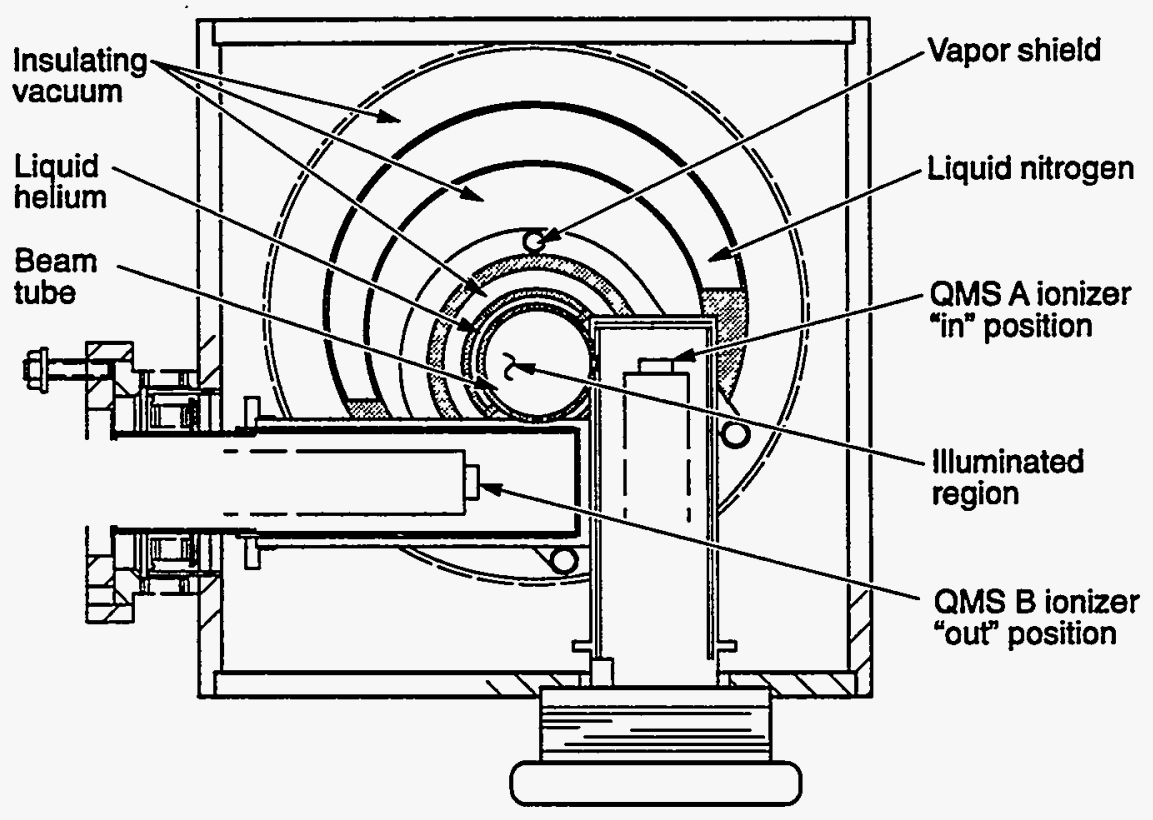

TIP-05743

Figure 4. CDG cold tube photodesorption apparatus: ${ }^{16}$ (a) cryostat, (b) detectors.

Four types of information are available from the experiment shown in Figure 4:

1. photodesorption coefficients of $\mathrm{H}_{2}, \mathrm{CO}$, and $\mathrm{CO}_{2}$ versus integrated photon flux with the beam tube at room temperature;

2. beam tube density (mostly $\mathrm{H}_{2}$ ) versus integrated photon flux with the beam tube at $4.5 \mathrm{~K}$; 
3. axial profile of the RGA $\mathrm{H}_{2}$ signal as the edge of the photon beam is moved across the detector;

4. time decay of the chopped RGA $\mathrm{H}_{2}$ signal.

Stainless steel and electrodeposited stainless steel tubes were investigated and found to have essentially the same behavior. The photodesorption coefficients of tightly bound gas were not measured in beam tube geometry. Rather they were measured in open geometry with samples cut from the beam tube. The open geometry experiments are reported in Reference 17. There was a systematic tendency for the $\mathrm{H}_{2}$ photodesorption coefficients at $4.5 \mathrm{~K}$ to exceed the values at room temperature by a factor $\sim 2$. This is perhaps not significant due to the experimental difficulties, and for purposes here we will ignore this difference. The maximum integrated photon flux in the CDG experiments was $\sim 10^{21}$ photons/m, corresponding to a day of SSC operation at design intensity.

We now present an analysis of the CDG experimental data for $\mathrm{H}_{2}$ in terms of the parameters of our vacuum model. Figure 5 shows the room-temperature photodesorption coefficients measured with a copperplated beam tube. ${ }^{16}$ The $\mathrm{H}_{2}$ data for $10^{19}<\Gamma<10^{21}$ photons/m are reasonably well represented by

$$
\eta\left(\mathrm{H}_{2}\right)=\frac{0.017}{\left(\frac{\Gamma}{\Gamma_{0}}\right)^{0.3}},
$$

where $\Gamma_{0}=10^{19}$ photons $/ \mathrm{m}$. Equation (52) is plotted as the dashed line in Figure 5. The other gases, $\mathrm{CO}$ and $\mathrm{CO}_{2}$, have similar power law dependence on integrated photon flux but lower magnitudes.

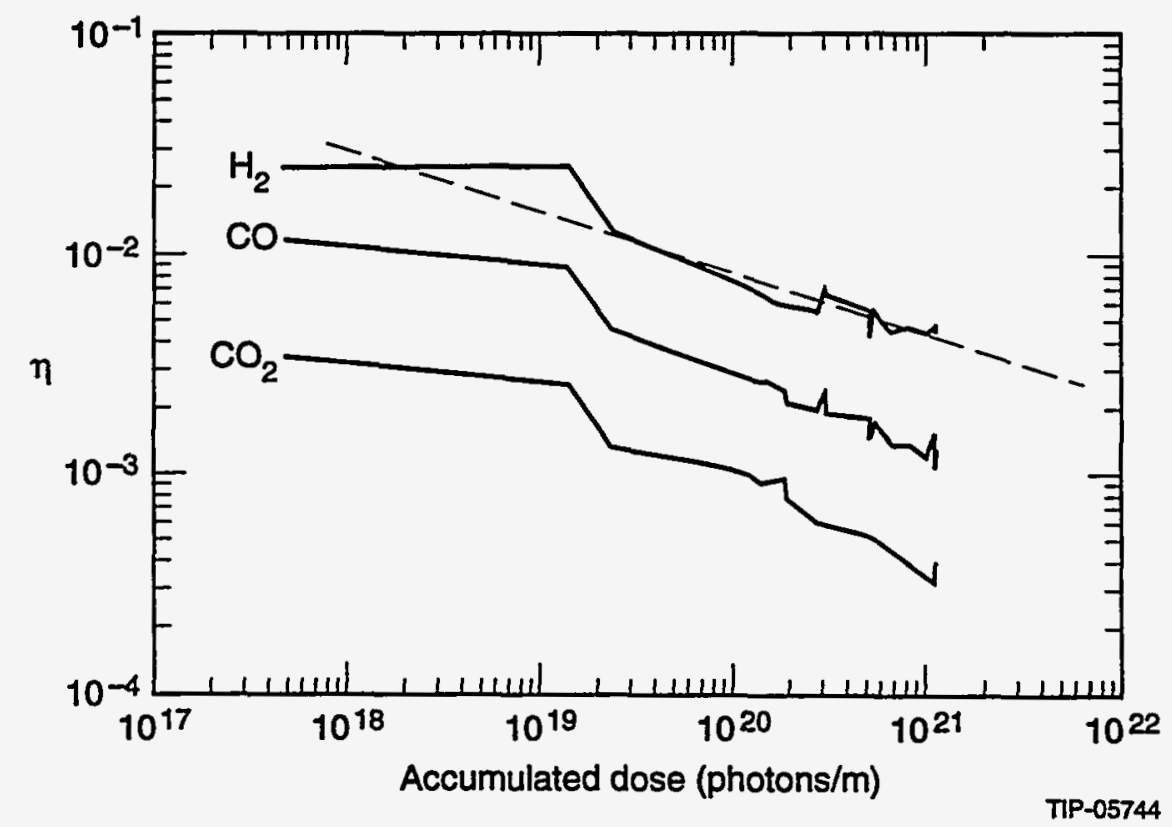

Figure 5. Room-temperature photodesorption data from the CDG experiment. ${ }^{16}$ 
Figure 6 shows the time behavior of the chopped $\mathrm{H}_{2}$ pulse recorded by the RGA; the $1 / e$ decay time is $\tau=0.235$ ms. From Eq. (13), $V \frac{\partial n}{\partial t}=-\sigma_{w} S_{w} n$, so $n(t)=n(0) e^{-\frac{t}{\tau}}$ with $\tau=\frac{2 a}{\sigma_{w} \bar{v}}$. Inserting the decay time and $a=1.6 \mathrm{~cm}$ we obtain $\sigma_{w} \bar{v}=1.36 \times 10^{4} \mathrm{~cm} / \mathrm{s}$. Information on the sticking coefficient can be gotten from the decrease in beam tube density as the edge of the photon beam is moved across the detector. If we let $x=0$ be the edge of the photon beam with $\dot{\Gamma}>0$ for $x<0$, and $\dot{\Gamma}=0$ for $x>0$, the solutions to Eq. (13) are

$$
\begin{aligned}
& n=\frac{\left(\eta_{1}+\eta^{\prime}\right) \dot{\Gamma}}{\sigma_{w} S_{w}}\left(1-\frac{1}{2} e^{\frac{x}{\lambda}}\right), \quad x<0 \\
& n=\frac{\left(\eta_{1}+\eta^{\prime}\right) \dot{\Gamma}}{\sigma_{w} S_{w}}\left(\frac{1}{2} e^{-\frac{x}{\lambda}}\right), \quad x>0,
\end{aligned}
$$

where $\lambda=\left(\frac{4}{3} \frac{a^{2}}{\sigma_{w}}\right)^{1 / 2}$.

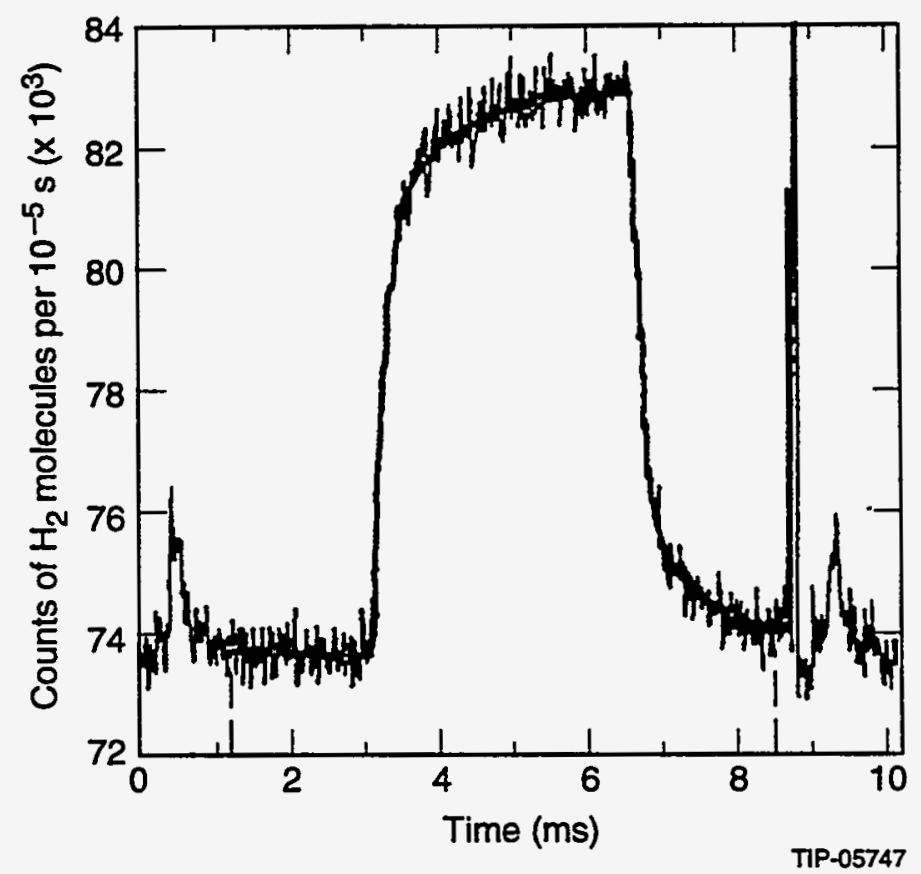

Figure 6. Time spectrum of an RGA $\mathrm{H}_{2}$ signal from the CDG experiment. ${ }^{34}$

Measurements of the spatial profile of the $\mathrm{H}_{2}$ density at the edge of the photon beam are given in Figure 7 of Reference 16. The data can be fit with $\lambda=6.75 \mathrm{~cm}$, corrected for smearing of the photon edge by the horizontal width of the electron beam in the BNL UV ring. Inserting this 
into the equation for $\lambda$ and with beam tube radius $a=1.6 \mathrm{~cm}$, we obtain $\sigma_{w}=0.075$. Returning to the result from the decay time of the chopped $\mathrm{H}_{2}$ signal, we then obtain for the mean speed of the dynamic component of beam tube density $\bar{v}=1.36 \times 10^{4} / \sigma_{w}=1.8 \times 10^{5} \mathrm{~cm} / \mathrm{s}$. This corresponds to an effective temperature $\mathrm{T} \sim 300 \mathrm{~K}$, far higher than molecules in equilibrium with the beam tube.

We now turn to the determination of $\eta^{\prime}$, the desorption coefficient of physisorbed $\mathrm{H}_{2}$. Figure 7 shows a typical plot of the dynamic $\mathrm{H}_{2}$ density versus integrated photon dose with the beam tube at $4.2 \mathrm{~K}^{34}$ The density is expressed in room temperature $\mathrm{T}=294 \mathrm{~K}$ pressure units and normalized to the photon flux in units of $2 \times 10^{14}$ photons $/ \mathrm{m} / \mathrm{s}$. Compared to room-temperature photodesorption experiments, the most striking thing is that the beam tube pressure is increasing with integrated photon flux, presumably due to the accumulation of physisorbed hydrogen. If we assume $\eta^{\prime} \gg \eta_{1}$, which will be justified a posteriori, then according to Eq. (26) the dynamic density increase, $\Delta n$, normalized to the photon flux is

$$
\frac{\Delta n}{\dot{\Gamma}}=\frac{\eta^{\prime}}{\sigma_{w} S_{w}}=\frac{\eta_{0}^{\prime}\left(s / s_{m}\right)}{\sigma_{w} S_{w}}=\frac{\eta_{0}^{\prime}}{\sigma_{w} S_{w}} \frac{\eta \Gamma}{A_{w} s_{m}},
$$

and the data in Figure 7 can be used together with the measured $\sigma_{w}$ and $\bar{v}$ to obtain estimates of $\eta^{\prime}$ versus integrated photon flux $\Gamma$. For example, for the data point at $\Gamma=5 \times 10^{20}$ photons/m we obtain $\eta^{\prime}=0.34 \mathrm{H}_{2}$ molecules/photon, alarmingly large compared to the tightly bound desorption coefficients in Figure 5 and Table 4 . It is clear that something quantitatively different from desorption at room temperature is taking place. To proceed further, the tightly bound desorption coefficient given by Eq. (52) can be used to calculate the surface density $s$ of physisorbed $\mathrm{H}_{2}$ as a function of $\Gamma$, assuming negligible axial diffusion. The data can then be used to estimate $\eta_{0}^{\prime}$ according to the last expression given in Eq. (55). This is an indirect procedure since the surface density was not measured in the experiment, but it is interesting to see what happens. The parameter $\eta_{0}^{\prime}$ can be estimated from a single data point, and then $n / \dot{\Gamma}$ can be plotted versus $\Gamma$ to see how well it reproduces the trend of increasing pressure with $\Gamma$. Using a normalization monolayer surface density $s_{m}=3 \times 10^{15} \mathrm{H}_{2} / \mathrm{cm}^{2}$, the dashed line drawn in Figure 7 is for $\eta_{0}^{\prime}=0.3$, and the agreement with the data is not too bad for the "first cycle" except at the first data point at $\Gamma=10^{18}$ photons $/ \mathrm{m} / \mathrm{s}$. A similar analysis of another data set gave $\eta_{0}^{\prime}=0.7$. The message here is that the desorption coefficient of physisorbed $\mathrm{H}_{2}$ can be very large, of the order of $0.5 \mathrm{H}_{2}$ molecules per incident photon as $s_{m}=3 \times 10^{15} \mathrm{H}_{2} / \mathrm{cm}^{2}$ is approached. Further discussion of the implications of this data are deferred until Section 6 , where we describe model calculations of beam tube vacuum. However, we note the remarkable fact that from this first experiment we have most of the parameters required by the model equations in Section 2 for predicting beam tube vacuum performance for $\mathrm{H}_{2}: \eta\left(=\eta_{1}+\eta_{2}\right), \eta^{\prime}, \sigma_{w}$, and $\bar{v}$. What is missing is information on the $\mathrm{H}_{2}$ isotherm and data for long-term exposures to photon flux. 


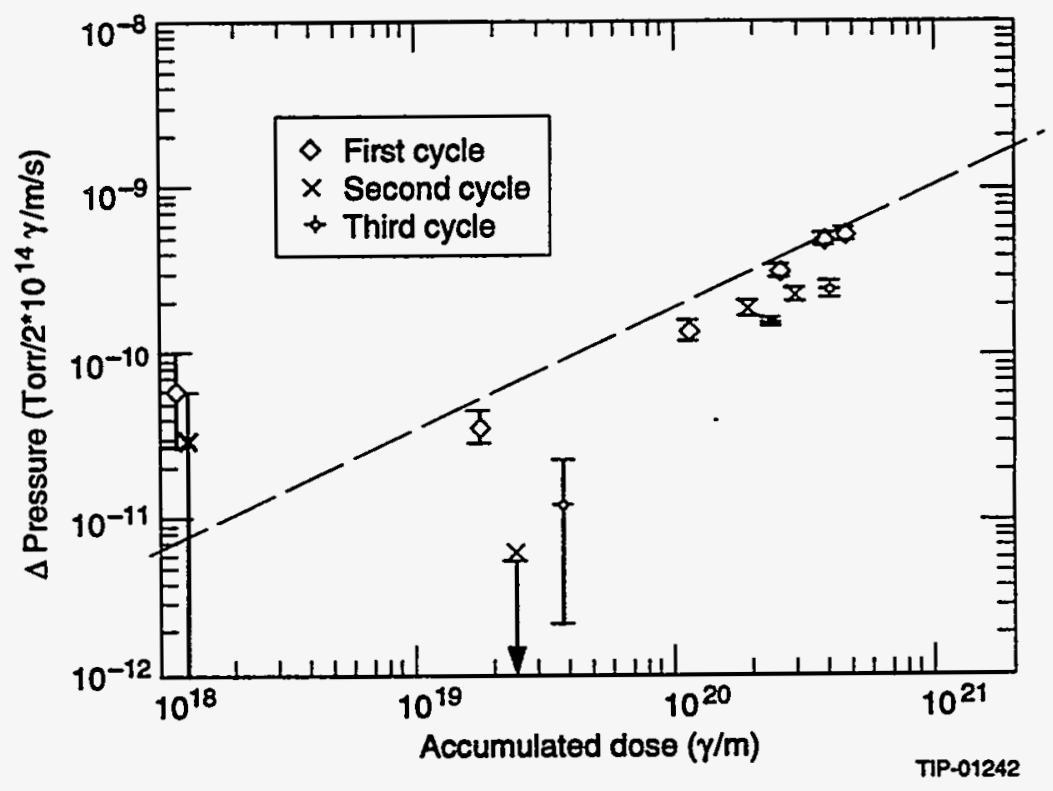

Figure 7. Dynamic $\mathrm{B}_{2}$ pressure versus integrated photon flux for the CDG experiment. ${ }^{34}$

\subsubsection{The BINP Experiments}

The second series of cold beam tube photodesorption experiments was initiated in 1991 following an important and influential re-examination of the expected vacuum behavior of the SSC by A. Maschke. ${ }^{35}$ The experiments were done as a collaboration between the SSCL and BINP in Russia. Several recent reports have been written on this work. ${ }^{36-40} \mathrm{~A}$ schematic of the apparatus is shown in Figure 8. The experiments were performed on a synchrotron radiation beamline of the VEPP2M electron-positron storage ring at BINP. Electron beam energy and current were set to produce the photon-critical energy and intensity of the SSC $\left(284 \mathrm{eV}, \sim 10^{16}\right.$ photons $\left./ \mathrm{m} / \mathrm{s}\right)$. A second synchrotron radiation beamline was constructed with approximately eight times higher intensity for high integrated flux experiments. So far two types of experiments have been done: the first with a simple beam tube (for measurement of $\eta$ ) and the second with a perforated liner configuration (for measurement of $\eta^{\prime}$ ). The simple beam tube and the bore tube liner were 1-m-long sections of electrodeposited $\mathrm{Cu}$ on stainless steel (ID $=32 \mathrm{~mm}$, $\mathrm{OD}=34.9 \mathrm{~mm}, \mathrm{Cu}$ thickness $=70 \mathrm{~mm}$ ). The liner was perforated with 6002 -mm-diameter holes spaced $1 \mathrm{~cm}$ axially and $60^{\circ}$ azimuthally. The bore tube outside the liner was stainless steel (ID $=41.9 \mathrm{~mm}, \mathrm{OD}=44.5 \mathrm{~mm}$ ) welded to the liner with annular rings at the ends. The simple beam tube and the liner bore tube were in turn welded into a horizontal LHe cryostat $(\sim 201 \mathrm{LHe})$ and formed the interface between $\mathrm{LHe}$ and vacuum. The beam tube was placed at an angle of $10 \mathrm{mrad}$ to the synchrotron radiation. Measurements of photon intensity and power at the end of a 1 - $\mathrm{m}$ beam tube indicated that only $\sim 5 \%$ of the incident photons and $\sim 0.5 \%$ of the incident power were reflected out the end. The temperature of the liner was not measured. The estimated temperature rise at the center of the liner is $5-10 \mathrm{~K}$ above the LHe temperature when exposed to a photon intensity of $125-250 \mathrm{~mW} / \mathrm{m}$. 
Gas densities were measured with calibrated if quadrupole RGAs at room temperature. An RGA was connected to the center of the beam tube and at each warm end. The center RGA viewed the beam tube through a 2.4-cm-diameter hole. Care was taken to avoid $4.2-\mathrm{K}$ cryosorbing surfaces in the tube connecting the RGA to the beam tube. The connecting tube had a temperature of $77 \mathrm{~K}$ at the beam tube hole and made a transition through thin stainless steel bellows to $294 \mathrm{~K}$ at the RGA. An annular vacuum gap of $\sim 0.2 \mathrm{~mm}$ separated the $77-\mathrm{K}$ viewing tube from the $4.2-\mathrm{K}$ beam tube. Thin-wall stainless steel bellows were used at the ends of the $4.2-\mathrm{K}$ beam tube for transitions to $77 \mathrm{~K}$ and $294 \mathrm{~K}$. The $294-\mathrm{K}$ vacuum ends of the cryostat were pumped with combination ion and titanium sublimation pumps.
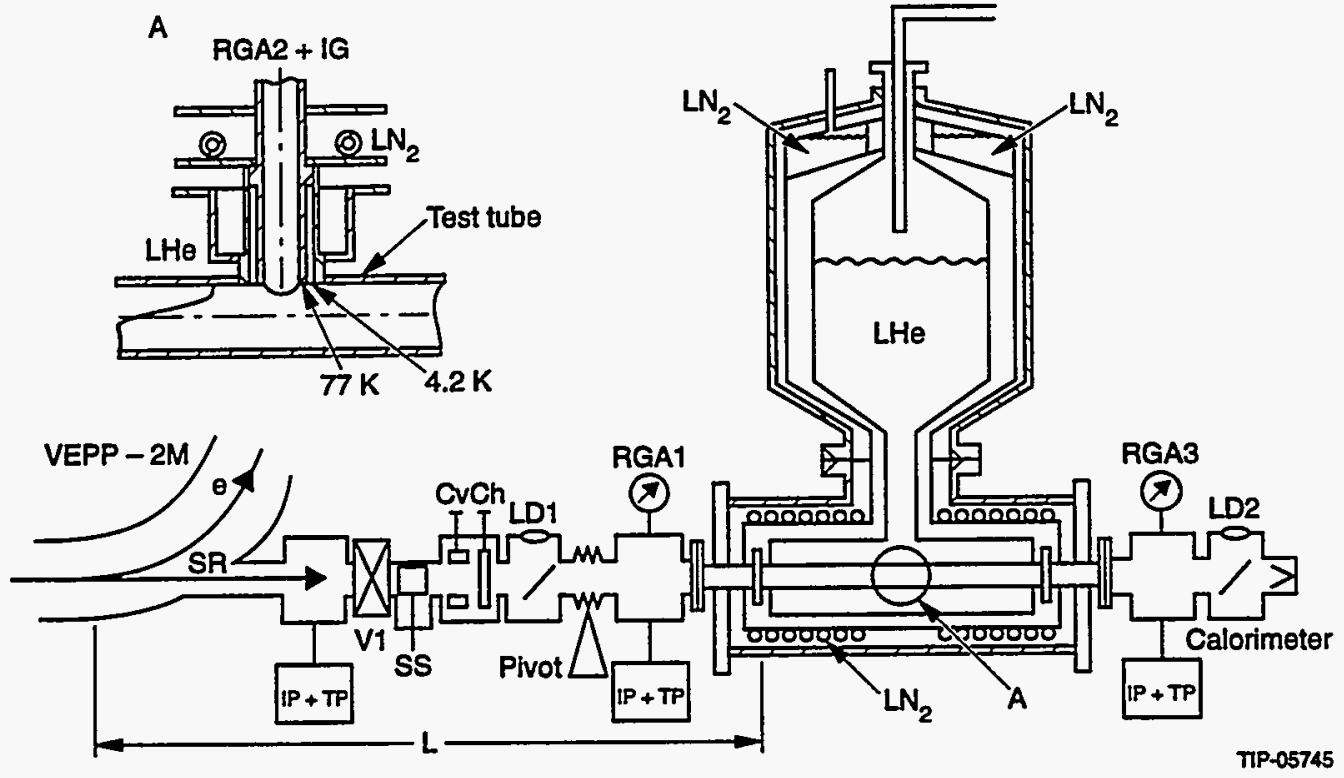

Figure 8. Schematic of the BINP apparatus for cold beam tube photodesorption experiments. ${ }^{39}$

The 77-K portion of the RGA viewing tube allows only cold-tube measurements of the photodesorption coefficients of $\mathrm{H}_{2}$ and $\mathrm{CO}$. It is planned to modify the viewing tube so that it has a temperature of $294 \mathrm{~K}$ along its entire length, and cold-tube photodesorption data will also be available for $\mathrm{CO}_{2}$ and $\mathrm{CH}_{4}$.

In contrast to the CDG setup in Figure 4(b), the RGA is not closely coupled to the beam tube, and the molecules make many wall collisions before reaching the ionizer. Assuming equilibration of molecular fluxes between the room-temperature RGA (RT $\approx 294 \mathrm{~K}$ ) and the cold beam tube (BT), we have: $n_{R T} \bar{v}_{R T}=n_{B T} \bar{v}_{B T}$. The RGA measures $n_{R T}$, and we may assume the molecules inside the RGA ionizer have had a large enough number of wall collisions that their velocity distribution is in equilibrium with room temperature $\left(\bar{v}_{R T}=1.8 \times 10^{5} \mathrm{~cm} / \mathrm{s}\right.$ for $\mathrm{H}_{2}$ ). The RGA measurement in the BINP experiment is then equivalent to a measurement of the product of density and mean molecular speed inside the cold tube. Although the cold beam tube density isn't measured directly as with the CDG setup, there is a gain in the sen- 
sitivity, which is proportional to the density ratio, $S=\frac{n_{R T}}{n_{B T}}=\frac{\bar{v}_{B T}}{\bar{v}_{R T}}$. For the CDG case this ratio was $S \approx \frac{1}{78}$ to $\frac{1}{150}$. For the BINP experiment this ratio depends on the density component. For the $\mathrm{H}_{2}$ isotherm density the mean molecular speed corresponds to $T=4.2 \mathrm{~K}$ and $S=\frac{1}{8.4}$. For the photodesorbed molecules, the mean thermal velocity from the CDG experiment is $\bar{v}_{B T}=1.8 \times 10^{5} \mathrm{~cm} / \mathrm{s}$, and from a recent experiment at BINP $\bar{v}_{B T}=8 \times 10^{4} \mathrm{~cm} / \mathrm{s},{ }^{40}$ and $S \approx 0.5-1$. The determination of the mean thermal velocity at BINP was accomplished with a hydrogen-ion beam method of direct density measurement inside the cold beam tube, described briefly in Section 7.1.

The BINP experimental measurements of photodesorption coefficients in a $4.2-\mathrm{K}$ electrodeposited $\mathrm{Cu}$ beam tube (ID $=32 \mathrm{~mm}$, length $=1 \mathrm{~m}$ ) have been described in detail in a recent report. ${ }^{37}$ Here we summarize some of the primary results. In a simple beam tube we have seen that if the desorption coefficient of physisorbed molecules $\left(\eta^{\prime}\right)$ is large compared to the desorption coefficient of tightly bound atoms and molecules $(\eta)$, then the difference density $\Delta n$ with photons on minus photons off is related to $\eta^{\prime}$ by

$$
\eta^{\prime} \dot{\Gamma}=\sigma_{w} A_{w} \frac{\bar{v}}{4} \Delta n
$$

Therefore, since $\bar{v} * \Delta n$ is conserved between the beam tube and RGA, the RGA measurements are equivalent to a measurement of $\eta^{\prime} / \sigma_{w}$. The photodesorption coefficient $\eta^{\prime} / \sigma_{w}$ for $4.2-\mathrm{K}$ physisorbed $\mathrm{H}_{2}$ is shown versus integrated photon flux in Figure 9(a). The isotherm density was measured separately with the photons gated off (see discussion in Section 4.1). This has been used to infer the surface density of physisorbed $\mathrm{H}_{2}$ and $\eta^{\prime} / \sigma_{w}$ versus $s$ in Figure 9(b). The data are reasonably well represented by a linear dependence: $\eta^{\prime}=\eta_{0}^{\prime}\left(s / s_{m}\right)$ with $\eta_{0}^{\prime} / \sigma_{w}=7.0 \pm 1.5$ and $s_{m}=3 \times 10^{15} \mathrm{H}_{2} / \mathrm{cm}^{2}$. It is impressive how large $\eta^{\prime}$ can be for reasonable estimates of $\sigma_{w}$ (say, $\sigma_{w}=0.075, \eta_{0}^{\prime}=0.5$ ). Changing to the liner configuration, the photodesorption coefficients for the tightly bound molecules at LHe temperature are related to the density by

$$
\eta \dot{\Gamma}=p N_{h} A_{h} \frac{\bar{v}}{4} n,
$$

where the transmission probability $p=0.59, N_{h}=600 \mathrm{holes} / \mathrm{m}$, and $A_{h}=0.01 \pi \mathrm{cm}^{2}$. Compared to Eq. (56), the known transmission coefficient takes the place of the sticking coefficient, and since once again $\bar{v} * n$ is conserved between the beam tube and RGA, the RGA measurements are equivalent to a measurement of $\eta$. The tightly bound photodesorption coefficients for $\mathrm{H}_{2}$ and $\mathrm{CO}$ measured this way are given in Figure 10 up to a photon flux of $10^{22}$ photons $/ \mathrm{m}$. Data are shown for 4.2-K and 294- $\mathrm{K}$ experiments. The photodesorption coefficients of tightly bound molecules in Figure 10 show an overall trend of decreasing with increasing photon exposure at room temperature and at $4.2 \mathrm{~K}$. The room-temperature coefficients appear somewhat larger than $4.2-\mathrm{K}$ coefficients. However, except for what appear to be initial temperature-dependent transients that are most apparent in CO in Figure 10, more careful experiments would be required to determine if this is a real effect or is due to other differences between the experiments. The two experiments were done with different samples of beam tube, and the room-temperature 
data was taken in the high-intensity beamline with eight times higher photon intensity than the 4.2-K data. The total numbers of desorbed molecules are estimated by integrating the desorption coefficients over the measured range of integrated photon flux $\Gamma=10^{22}$ photons $/ \mathrm{m}$ : for the $4.2-\mathrm{K}$ data, $1.1 \times 10^{19} \mathrm{H}_{2} / \mathrm{m}$ and $9.2 \times 10^{17} \mathrm{CO} / \mathrm{m}$; for the $294-\mathrm{K}$ data, $3 \times 10^{19} \mathrm{H}_{2} / \mathrm{m}$ and $5 \times 10^{18} \mathrm{CO} / \mathrm{m}$.
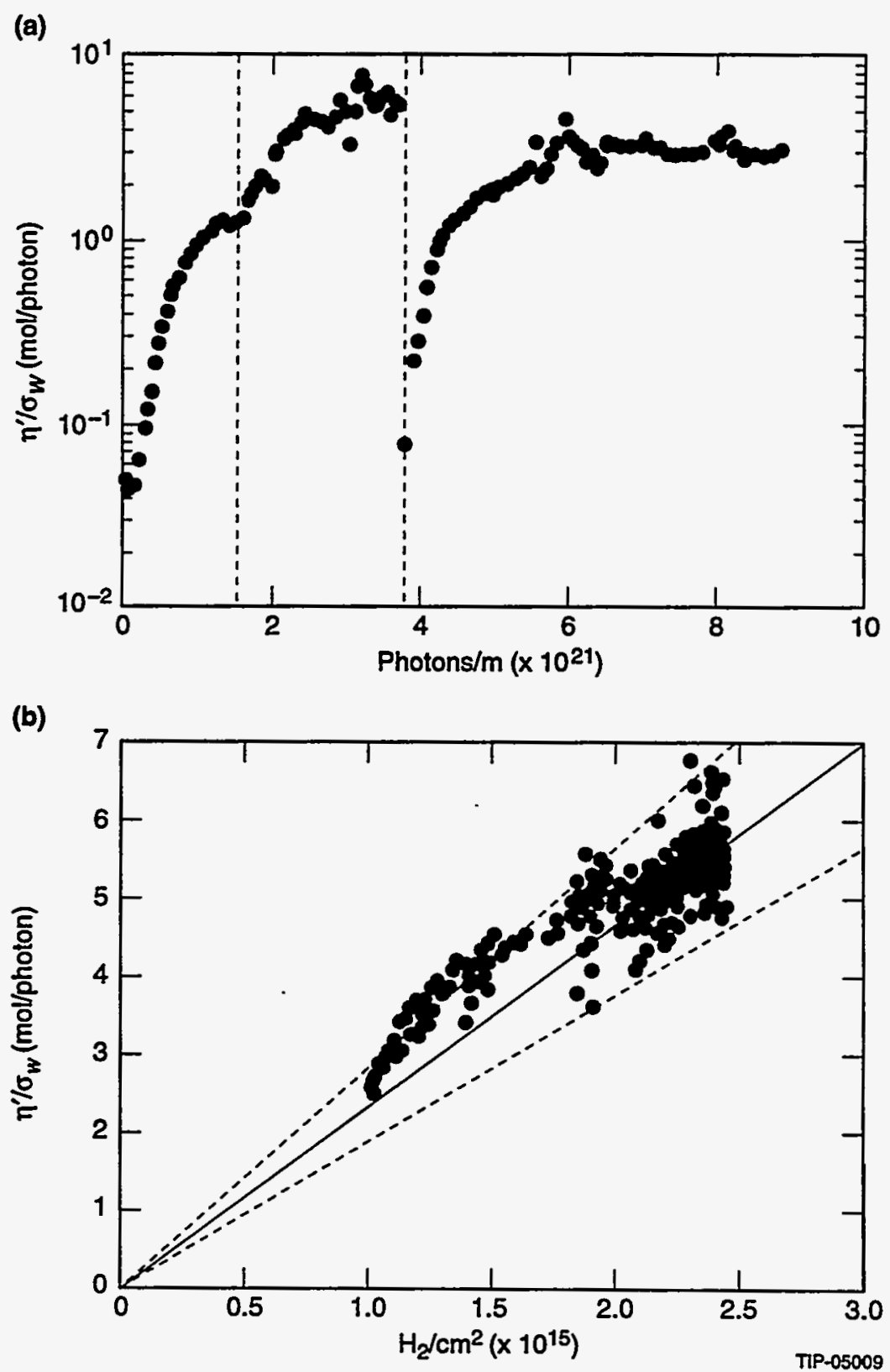

Figure 9. Photodesorption coefficient $\eta^{\prime} / \sigma_{w}$ for physisorbed $\mathrm{H}_{2}$ measured at BINP versus (a) integrated photon flux, (b) surface density of physisorbed $\mathrm{H}_{2} \cdot{ }^{37}$ 


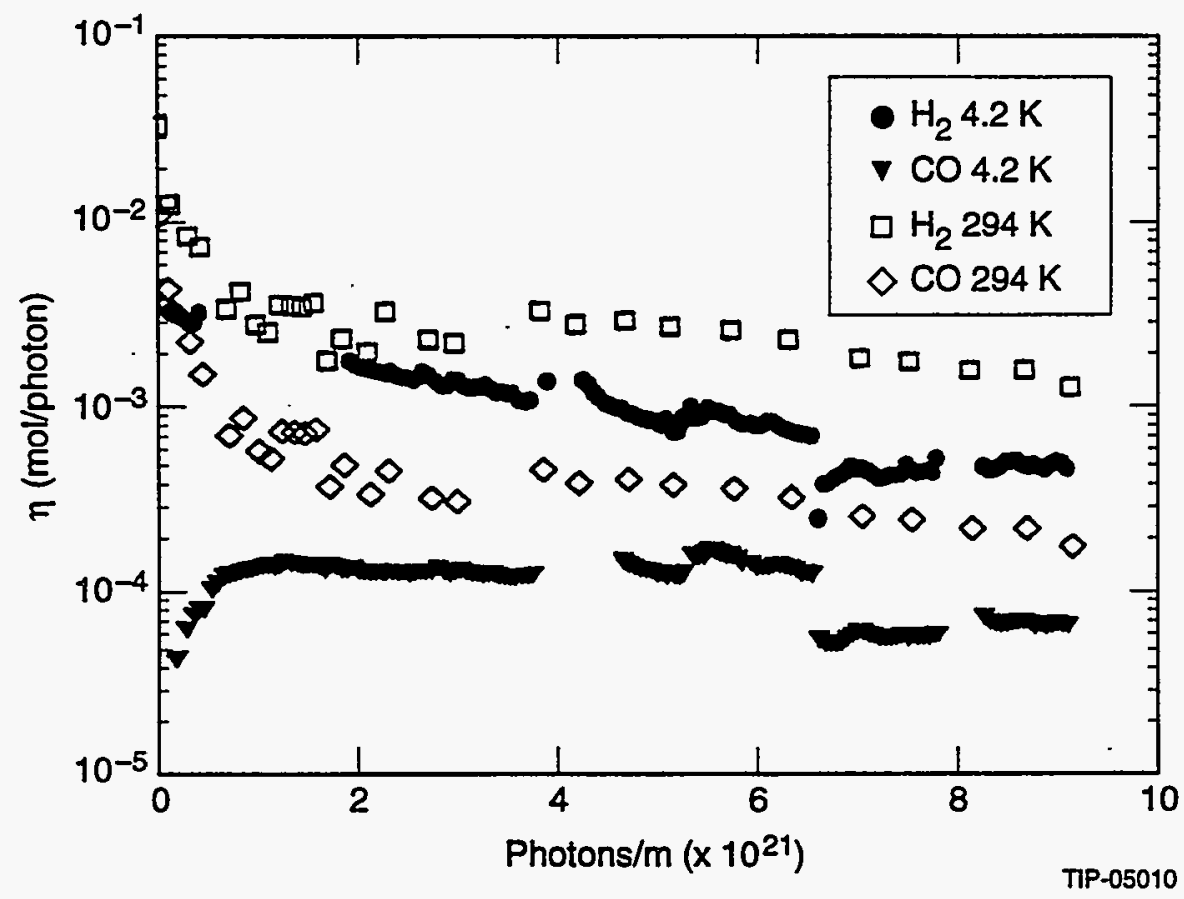

Figure 10. $\eta\left(\mathrm{H}_{2}\right)$ and $\eta(\mathrm{CO})$ versus integrated photon flux for electroplated $\mathrm{Cu}$. Data are shown for 4.2-K and 294-K experiments. ${ }^{37}$

For a simple 4.2- $\mathrm{K}$ beam tube without a liner, we can estimate the transition photon flux $\Gamma$ when the photodesorption of physisorbed $\mathrm{H}_{2}$ molecules dominates photodesorption of tightly bound molecules. Since $\eta^{\prime}=\eta_{0}^{\prime}\left(\frac{s}{s_{m}}\right) \approx \eta_{0}^{\prime}\left(\frac{\eta \Gamma}{A_{w} s_{m}}\right)$, the condition $\eta=\eta^{\prime}$ yields $\Gamma=\frac{A_{w} s_{m}}{\eta_{0}^{\prime}}$. We note that this estimate of the integrated flux at which photodesorption of physisorbed molecules surpasses tightly bound molecules is independent of the magnitude of $\eta$. Inserting numbers, $\eta_{0}^{\prime}=0.5, A_{w}=1005 \mathrm{~cm}^{2} / \mathrm{m}$, and $s_{m}=3 \times 10^{15} \mathrm{H}_{2} / \mathrm{cm}^{2}$, we obtain the transition flux $\Gamma=6 \times 10^{18}$ photons $/ \mathrm{m}$. For the experimental conditions $\dot{\Gamma}=1 \times 10^{16}$ photons $/ \mathrm{m} / \mathrm{s}$, the transition time is $\sim 10 \mathrm{~min}$. Dominance by photodesorption of physisorbed molecules in a simple beam tube at LHe temperature occurs very quickly after the first exposure to photons. The surface density of physisorbed $\mathrm{H}_{2}$ at which the transition occurs is a small fraction of a monolayer and actually depends on the magnitude of $\eta: \frac{s}{s_{m}}=\frac{\eta \Gamma}{A_{w} s_{m}}=0.008$ for the initial $\eta\left(\mathrm{H}_{2}\right)=4 \times 10^{-3}$. The fact that the transition occurs so early in time justifies neglecting axial diffusion and assuming $\eta$ is constant in this analysis.

For the case of the liner experiment we can use Eq. (38) to estimate the surface density of $\mathrm{H}_{2}$ physisorbed on the liner:

$$
\begin{aligned}
\frac{s}{s_{m}} & =\left(\frac{\sigma_{w}}{\eta_{0}^{\prime}}\right) \frac{A_{w}}{p N_{h} A_{h}} \eta=12.9 \eta \\
& =0.05 \text { for } \eta=4 \times 10^{-3} \\
& =0.005 \text { for } \eta=4 \times 10^{-4} .
\end{aligned}
$$


In Figure 11 we have plotted the cumulative and differential photon fluxes required to desorb successive monolayers of $\mathrm{H}_{2}$ (one monolayer $=A_{w} s_{m}=3.1 \times 10^{18} \mathrm{H}_{2} / \mathrm{m}$ ) for $4.2 \mathrm{~K}$ and $294 \mathrm{~K}$; more data are shown for $294 \mathrm{~K}$ because that experiment was continued to $2 \times 10^{23}$ photons $/ \mathrm{m}$. The differential data give the integrated photon flux required to desorb each successive monolayer, and the cumulative data give the total flux required to desorb $N$ monolayers. Since the time to form an $\mathrm{H}_{2}$ monolayer is an upper bound on the beam tube warm-up interval for a proton collider with a simple beam tube, this information is crucial for deciding the issue of liner or no liner for a collider such as the SSC. On the other hand, if a liner approach is adopted the information is necessary to estimate the required capacity of $\mathrm{H}_{2}$ cryosorber unless the cryostat operating temperature is so low that the saturated vapor pressure of $\mathrm{H}_{2}$ is not a concern, as in the LHC. The 4.2-K data in Figure 11 show that the first monolayer is desorbed with the equivalent of 1.4 days' integrated photon flux, and by the fourth monolayer this has increased to 7 days. Simple extrapolation of the trend of the data indicates that between 10 and 20 monolayers would be desorbed before reaching the level of one monolayer per operational year $\left(2 \times 10^{23}\right.$ photons $\left./ \mathrm{m}\right)$. Since this conditioning process would restart each time a section of the beam tube is replaced, this would seem to be an unacceptable burden on machine operation. Perhaps a better beam tube material or conditioning procedure can be found that reduces the desorbable $\mathrm{H}_{2}$. Several suggestions have been made. The experimental machinery is now in place to carry out comparative studies of such options. Of course this problem is avoided if a liner approach is used. This point was demonstrated in the experiments measuring the desorption coefficients in Figures 9 and 10; a liner was shown to effectively shield the cryosorbed molecules from the synchrotron radiation.

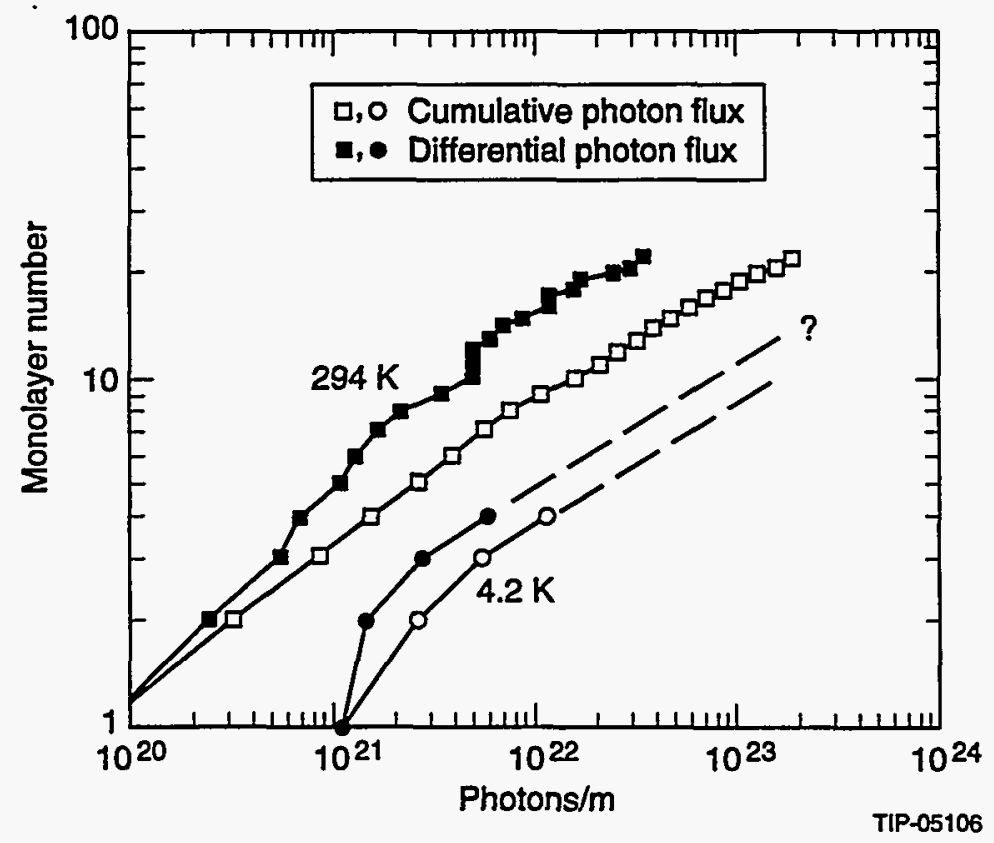

Figure 11. Number of monolayers of $\mathrm{H}_{2}$ desorbed from electrodeposited $\mathrm{Cu}$ versus cumulative and differential integrated photon flux. Data are shown for the $4.2-\mathrm{K}$ and $294-\mathrm{K}$ experiments. ${ }^{38}$ 
Before leaving this section we would like to discuss a simple calculation of the $\mathrm{H}_{2}$ molecules bouncing off the beam tube wall and slowing down until they stick. In this model the sticking coefficient is 0 for all wall collisions except the last, and then it is 1 . For a sticking coefficient $\sigma_{w}=0.075$ averaged over all collisions, the molecules make on the average about $N=13$ collisions with the wall before sticking. If they are born with energy $E_{0}$ and lose half their energy on each collision with the wall until they stick, then the mean speed can be shown to be

$$
\bar{v}=N \frac{2^{1 / 2}-1}{2^{N / 2}-1}\left(\frac{2 E_{0}}{m}\right)^{1 / 2} .
$$

Inserting $N=13$ and $\bar{v}=8.0 \times 10^{4} \mathrm{~cm} / \mathrm{s}$ gives an initial energy $E_{0}=1.9 \mathrm{eV}$ and an energy at sticking $E_{0} / 2^{N}=2.7 \mathrm{~K}$, which seem to be in reasonable ranges.

\subsubsection{Comparison of $C D G$ and BINP Experiments}

In this section we compare the BINP and CDG measurements of $\eta^{\prime}$ and $\eta$ at $4.2 \mathrm{~K}$ for $\mathrm{H}_{2}$. If we take $\sigma_{w}=0.075$ from the CDG data, then the BINP experiment gives $\eta_{0}^{\prime}=0.5 \pm 0.1$, which is in the middle of the range of 0.3-0.7 estimated for CDG data in Section 3.2.1. So the two experiments are consistent for the photodesorption of physisorbed $\mathrm{H}_{2}$. We now turn to comparison of desorption coefficients of tightly bound $\mathrm{H}_{2}$ at $4.2 \mathrm{~K}$, measured in the CDG open sample geometry experiments in Reference 17 and in the BINP quasi-closed beam tube liner geometry. There are legitimate reasons for questioning the validity of a comparison of photodesorption coefficients measured in open sample and beam tube experiments, including: (1) diffusely scattered photons contribute significantly to desorption from a beam tube surface but not from an open sample; (2) the detailed profiles of surface illumination intensity may vary greatly; (3) photon impact may create active pumping sights for tightly bound molecules; and (4) desorption coefficients for open samples are presented as a function of photons per unit area, whereas beam tube experiments use photons per unit length. Nevertheless we will compare the photodesorption coefficients of Figure 10 to the open sample results of Reference 17, assuming the beam tube is uniformly illuminated. With this assumption $10^{21}$ photons $/ \mathrm{m}$ is equivalent to $10^{18}$ photons $/ \mathrm{cm}^{2}$; this same assumption was made in Reference 17 for comparing $4.2-\mathrm{K}$ and room-temperature open sample data to room-temperature beam tube data in Reference 16. From Figure 10, $\eta\left(\mathrm{H}_{2}, 4.2 \mathrm{~K}\right)=2.5 \times 10^{-3}$ at $10^{21}$ photons $/ \mathrm{m}$. From Figure 6 in Reference 17, an average over three open samples gives $\eta\left(\mathrm{H}_{2}, 4.2 \mathrm{~K}\right)=3.8 \times 10^{-3}$ at $10^{18}$ photons $/ \mathrm{cm}^{2}$. The two results are within a factor of two; given the very different approaches, this is perhaps as good agreement as could be hoped for.

\section{Some Related Vacuum Experiments}

\subsection{The Hydrogen Isotherm}

In order to predict the behavior of the $\mathrm{H}_{2}$ isotherm density in Eq. (26) we need to know the isotherm density as a function of the surface density of adsorbed $\mathrm{H}_{2}$. The $4.2-\mathrm{K} \mathrm{H}_{2}$ isotherm density measured at BINP is shown in Figure 12 for an electrodeposited $\mathrm{Cu}$ beam tube. ${ }^{38}$ The data were obtained with the same cryostat used for the photodesorption experiments of Figure 8, but with an additional small-diameter, perforated dosing tube inserted along the axis 
of the beam tube. For the data shown, the $\mathrm{H}_{2}$ dosing rate was $2.6 \times 10^{12} \mathrm{H}_{2} / \mathrm{cm}^{2} / \mathrm{s}$, and the duration of the entire experiment $2.9 \times 10^{3} \mathrm{~s}$. A background density $5.0 \times 10^{8} \mathrm{H}_{2} / \mathrm{cm}^{3}$ has been subtracted from the raw data. The isotherm density increased to $4 \times 10^{8} \mathrm{H}_{2} / \mathrm{cm}^{3}$ at adsorbed surface density $2.3 \times 10^{15} \mathrm{H}_{2} / \mathrm{cm}^{2}$ and then increased over three orders of magnitude to reach a density $\sim 1.4 \times 10^{12} \mathrm{H}_{2} / \mathrm{cm}^{3}$ at adsorbed surface density $7.6 \times 10^{15} \mathrm{H}_{2} / \mathrm{cm}^{2}$. The experimental data in Figure 12 are reasonably well represented by the BET Eq. (15), with monolayer density $s_{m}=3 \times 10^{15} \mathrm{H}_{2} / \mathrm{cm}^{2}$, saturation density $n_{\text {sat }}(4.2 \mathrm{~K})=2.0 \times 10^{12} \mathrm{H}_{2} / \mathrm{cm}^{3}$, and $\alpha=1.3 \times 10^{4}$. The isotherm vapor density reaches $4 \times 10^{10} \mathrm{H}_{2} / \mathrm{cm}^{3}$, the local density limit allowable for the SSC design, in the neighborhood of the surface density $s_{m}=3 \times 10^{15} \mathrm{H}_{2} / \mathrm{cm}^{2}$. The dosing tube was removed prior to photodesorption experiments.

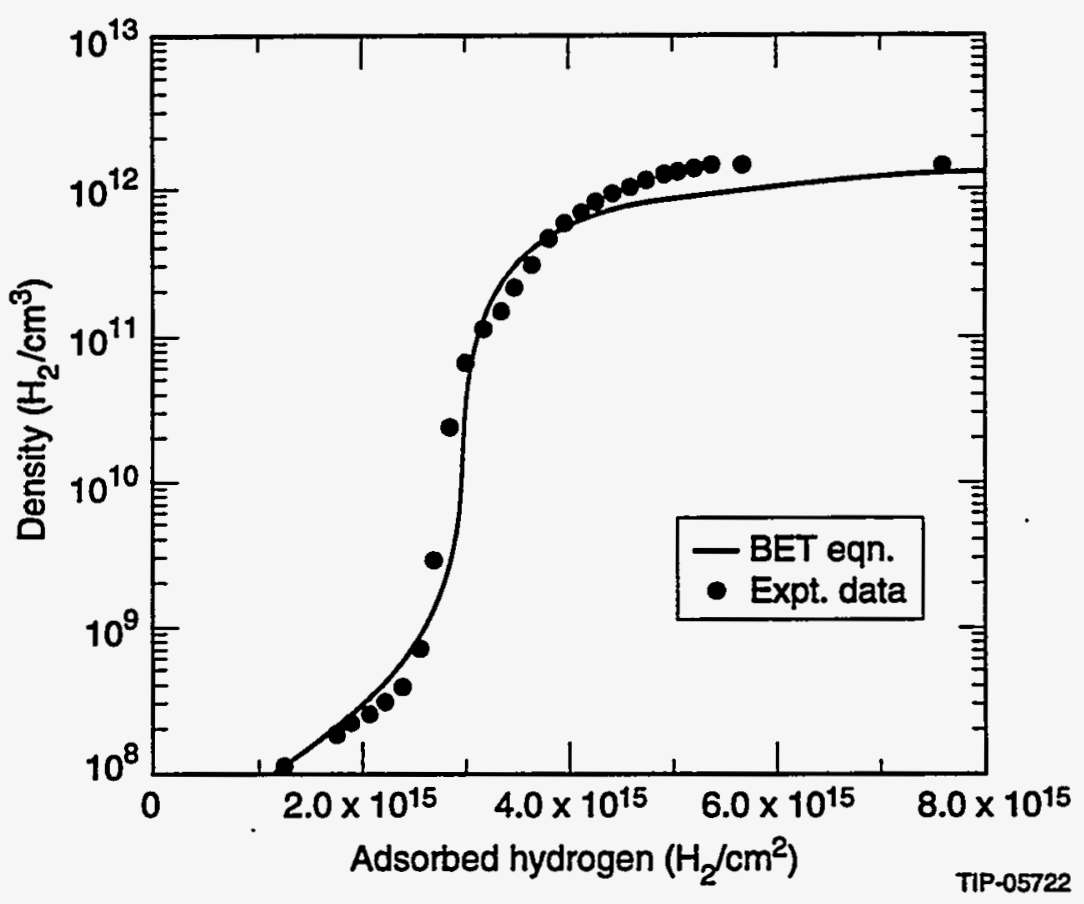

Figure $12 . \mathrm{H}_{2}$ isotherm density versus surface density on electrodeposited $\mathrm{Cu}^{38}$

It is interesting to use the parameters of the BET equation plotted in Figure 12 and the photodesorption measurement of $\eta^{\prime}$ in Figure 9 to estimate the sojourn times given by Eqs. (16) and (34). We will assume the sticking coefficient $\sigma_{w}^{t}=1$ for a thermalized $\mathrm{H}_{2}$ molecule at $4.2 \mathrm{~K}$ with mean speed $\bar{v}_{t}=2.1 \times 10^{4} \mathrm{~cm} / \mathrm{s}$. For the other parameters we take $\eta_{0}^{\prime}=0.075 \times 7.0=0.525$, $A_{w}=2 \pi(1.6)(100)=1005 \mathrm{~cm}^{2} / \mathrm{m}, \dot{\Gamma}=10^{16}$ photons $/ \mathrm{m} / \mathrm{s}$, and $s_{m}=3 \times 10^{15} \mathrm{H}_{2} / \mathrm{cm}^{2}$. These values correspond to the BINP cold-tube experiments and the anticipated operation of the SSC. The results for $\tau_{w}^{t}, \tau_{w}^{\eta^{\prime}}$ and the reciprocal sum $\tau_{w}$ are given in Figure 13. The photodesorption sojourn time is plotted beyond its known range of validity, but the generalized sojourn time is dominated by thermal desorption in that range anyway. At low surface coverage the sojourn time is dominated by photodesorption and is $\sim 500 \mathrm{~s}$; as the surface density builds up the thermal desorption time decreases and dominates as one monolayer is approached. At one monolayer 
$s=3 \times 10^{15} \mathrm{H}_{2} / \mathrm{cm}^{2}$, the sojourn time is $\sim 30 \mathrm{~s}$. The sojourn time continues to decrease, reaching a broad minimum $\sim 1 \mathrm{~s}$ at $s=2 s_{m}=6 \times 10^{15} \mathrm{H}_{2} / \mathrm{cm}^{2}$, and then slowly increases with increasing $s$.

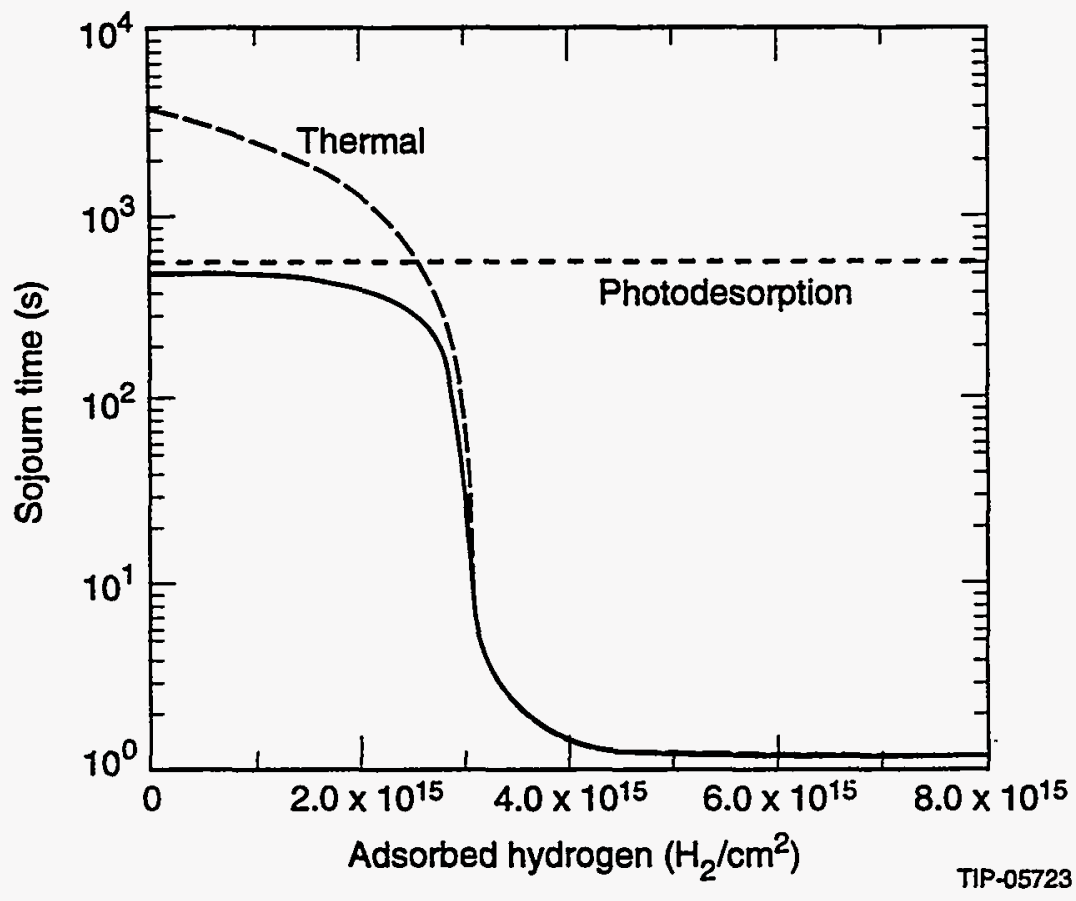

Figure $13 . \mathrm{H}_{2}$ sojourn time versus surface density.

The sojourn times have been used to calculate the axial diffusion times (Eq. (30)) for beam tube lengths of experimental interest: $L=1 \mathrm{~m}$ (photodesorption experiment), $L=5 \mathrm{~m}$ (superconducting quadrupole magnet), $L=15 \mathrm{~m}$ (superconducting dipole magnet), and $L=$ $90 \mathrm{~m}$ (length of a half cell of the accelerator lattice). Results are shown in Figure 14, where the physical parameters are the same as for Figure 13. The axial diffusion times decrease rapidly by a factor of $\sim 35$ in the neighborhood of one monolayer due to the large decrease in the thermal desorption sojourn time. This factor is less than the decrease in sojourn times in Figure 13 because the scaling factor $L^{2} / \lambda^{2}$ has a sticking coefficient in it that has been taken to be 0.075 for photodesorbed $\mathrm{H}_{2}$ and 1.0 for thermally desorbed $\mathrm{H}_{2}$. Since for an $L=1 \mathrm{~m}$ beam tube the initial axial diffusion time is $\sim 4 \mathrm{~h}$ and is short compared to the duration of the photodesorption experiments, it is clear that the effects of axial diffusion will be noticeable. This has been discussed together with the experimental data in Reference 37 and will be visited again in the model vacuum calculation in Section 6 . For the actual accelerator components the vacuum conditions inside the beam tube are isolated from the ends for very long times: $\sim 4$ days for a 5 -m quadrupole, $\sim 40$ days for a 15 -m dipole. 


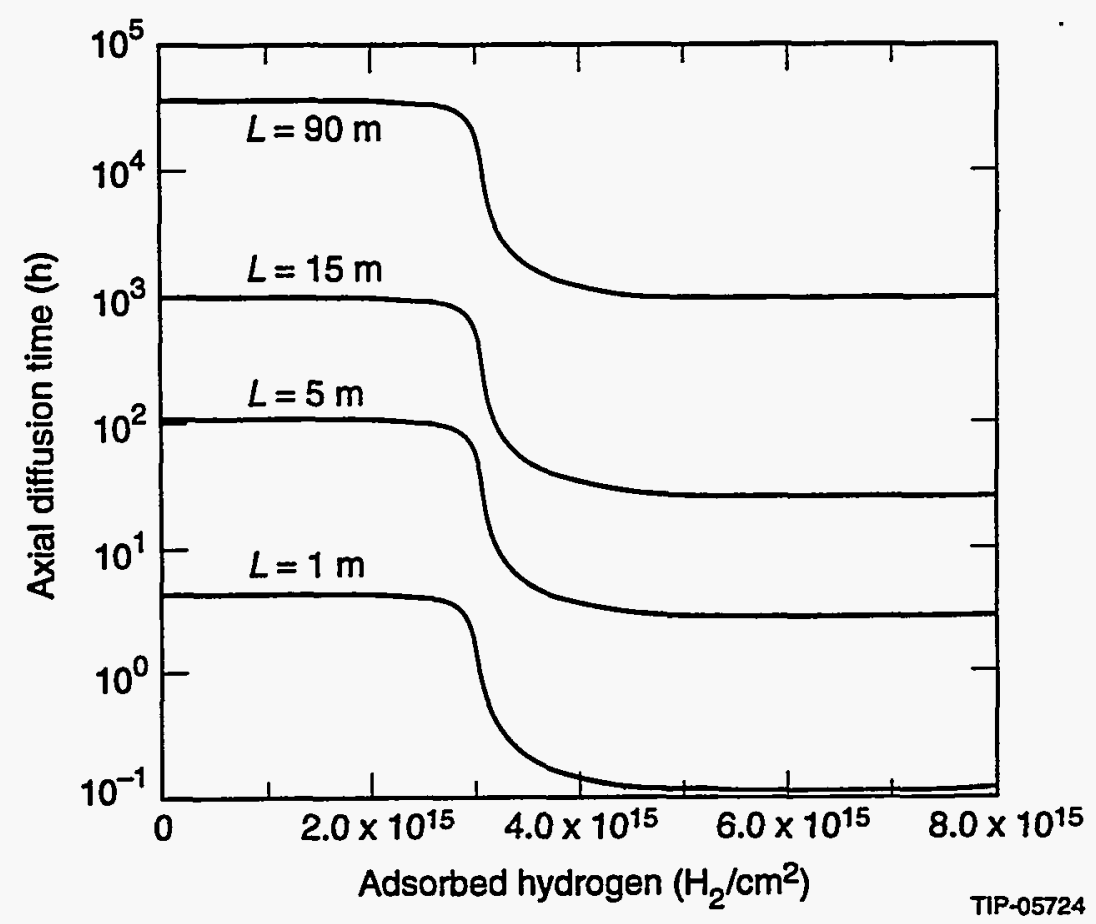

Figure 14. Axial diffusion times in beam tubes with length $L=1 \mathrm{~m}, 5 \mathrm{~m}, 15 \mathrm{~m}$, and $90 \mathrm{~m}$.

\subsection{The Hydrogen Depth Profile}

The 294- $\mathrm{K} \mathrm{H}_{2}$ photodesorption data in Figure 11 indicate approximately 20 monolayers $/ \mathrm{m}(1.8$ Torr $-1 / \mathrm{m})$ of $\mathrm{H}_{2}$ desorbed at $2 \times 10^{23}$ photons $/ \mathrm{m}$. The source of this $\mathrm{H}_{2}$ is a question of obvious interest. A first step in answering this question is to obtain the hydrogen depth profile of the surfaces we have studied. ${ }^{41}$ Such profiles have been obtained using nuclear reaction analysis (NRA) at the State University of New York (SUNY)-Albany Accelerator Laboratory. The particular reaction used is ${ }^{15} \mathrm{~N}+\mathrm{H} \longrightarrow{ }^{12} \mathrm{C}+{ }^{4} \mathrm{He}+\gamma(4.43 \mathrm{MeV})$. This reaction has a narrow resonance (width $\Gamma=1.8 \mathrm{keV}$ ) at $E_{\text {res }}=6.4 \mathrm{MeV}$, which is particularly effective for depth-profiling surface hydrogen density. A sample is irradiated with a monoenergetic ${ }^{15} \mathrm{~N}$ beam and the $\gamma$-ray intensity at $4.43 \mathrm{MeV}$ is measured with a bismuth germinate (BGO) scintillation detector. The depth profile is obtained by scanning the energy of the ${ }^{15} \mathrm{~N}$ beam. The samples irradiated have been cleaned with the standard cleaning procedure used for the photodesorption experiments. Figure 15 shows the hydrogen depth profile results for the Silvex electrodeposited $\mathrm{Cu}$ and, for comparison, for high-purity Hitachi 10100 bulk $\mathrm{Cu}$ (the last entry in Table 4). Both samples show a sharp peaking of hydrogen density $\sim 4 \times 10^{22} \mathrm{H} / \mathrm{cm}^{3}$ in a surface layer approximately $100 \AA$ thick decreasing to a density $\sim 1-2 \times 10^{20} \mathrm{H} / \mathrm{cm}^{3}$ in the bulk material. Compared to the bulk $\mathrm{Cu}$ atomic density $8.5 \times 10^{22} \mathrm{Cu} / \mathrm{cm}^{3}$, the fractional atomic percent of hydrogen is $0.1-0.2 \%$ below the surface layer. The observed thickness of the surface layer may be limited by the spatial resolution of the profiling method. Consequently the actual thickness may be less than $\sim 100 \AA$ and the $\mathrm{H} / \mathrm{cm}^{3}$ within the surface layer higher than shown in Figure 15 . 
However, the surface $\mathrm{H} / \mathrm{cm}^{2}$ obtained by integrating through the peak is given correctly. The surface densities obtained by integration are $2.3 \times 10^{16} \mathrm{H} / \mathrm{cm}^{2}$ for the Silvex electrodeposited $\mathrm{Cu}$, and $2.6 \times 10^{16} \mathrm{H} / \mathrm{cm}^{2}$ for the Hitachi 10100 high-purity $\mathrm{Cu}$. Considering the very different manufacturing processes for these materials it is perhaps surprising how similar these results are, even in the bulk material far below the surface.

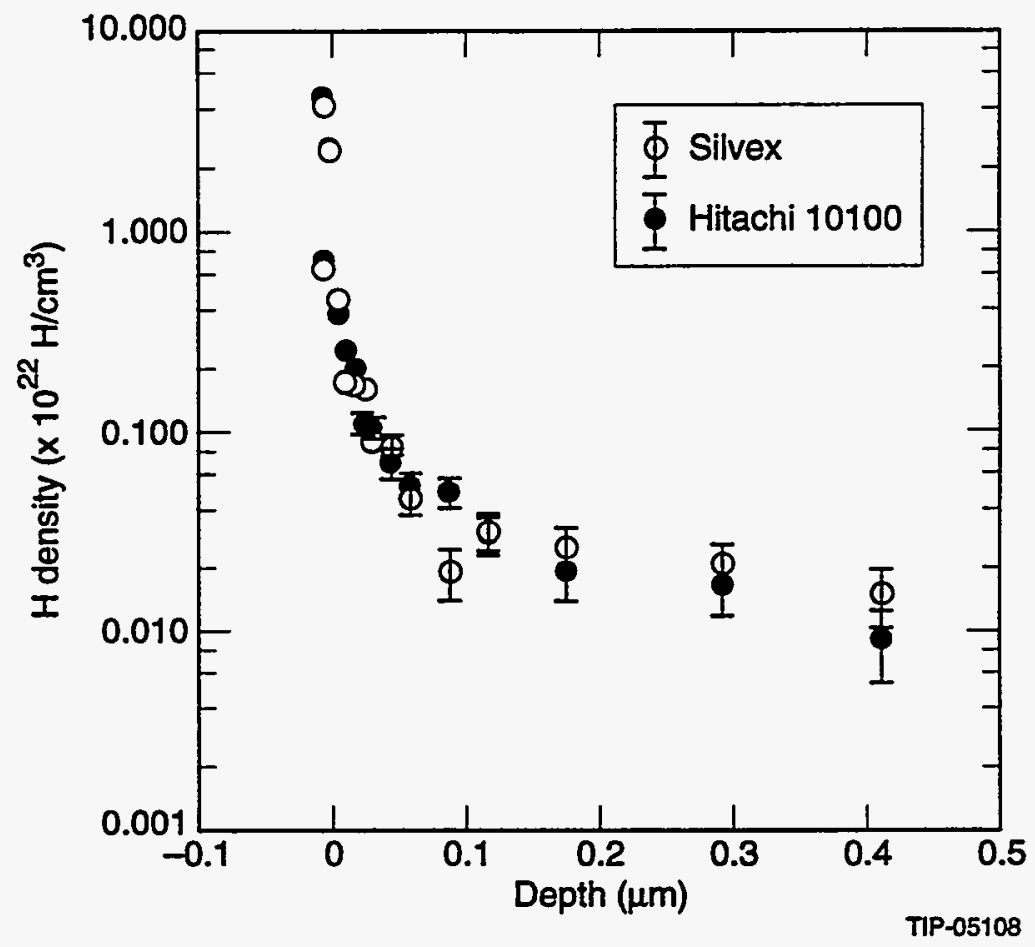

Figure 15. Depth profile of hydrogen in Silvex electrodeposited $\mathrm{Cu}$ and Hitachi 10100 high-purity bulk $\mathrm{Cu} .{ }^{41}$

Photodesorption data at room temperature have previously been obtained for both of the materials investigated in Figure 15 up to an integrated photon flux $\Gamma=10^{23}$ photons/m (Table 4 from Reference 20). It is interesting to compare the amount of photodesorbed hydrogen with the hydrogen surface densities measured with nuclear reaction analysis. This is done in Table 5. The photodesorption experiments measure an amount of molecular hydrogen desorbed per meter length of beam tube. To convert this to an amount of $\mathrm{H}_{2} / \mathrm{cm}^{2}$ that can be compared with the NRA analysis, we have simply divided by the beam tube area per meter $\left(=1005 \mathrm{~cm}^{2} / \mathrm{m}\right)$, ignoring the question of the uniformity of photodesorption. Two things are notable in Table 5: (1) the photodesorption and NRA data separately show similar results for Silvex electrodeposited $\mathrm{Cu}$ and Hitachi $10100 \mathrm{Cu}$, and (2) the amount of hydrogen in the surface layer measured with NRA analysis is a significant fraction $(0.35-0.5)$ of the hydrogen photodesorbed by $10^{23}$ photons $/ \mathrm{m}$. The depth-profile results reported in Reference 41 and the 
photodesorption experiments of a more extensive list of materials in Reference 20 suggest that all the materials investigated have a similar hydrogen-rich surface layer that is acquired during exposure to atmosphere and the cleaning process.

Table 5. Comparison of the 294-K photodesorption yield of hydrogen with the NRA analysis of hydrogen surface density.

\begin{tabular}{|l|c|c|}
\hline Material & $\begin{array}{c}\text { Photodesorbed molecular } \\
\text { hydrogen }\left(\mathrm{H}_{2}\right) \text { at } \\
\Gamma=10^{23} \text { photons } / \mathrm{m}\end{array}$ & $\begin{array}{c}\text { NRA surface density } \\
\text { of protons }(\mathrm{H})\end{array}$ \\
\hline Silvex elec. $\mathrm{Cu}$ & $\begin{array}{c}1.0 \text { Torr } 1 / \mathrm{m} \mathrm{H}_{2} \\
3.3 \times 10^{16} \mathrm{H}_{2} / \mathrm{cm}^{2}\end{array}$ & $2.3 \times 10^{16} \mathrm{H} / \mathrm{cm}^{2}$ \\
& 3.8 Torr $1 / \mathrm{m} \mathrm{H}_{2}$ & $2.6 \times 10^{16} \mathrm{H} / \mathrm{cm}^{2}$ \\
\hline Hitachi $10100 \mathrm{Cu}$ & $\begin{array}{c}0.8 \times 10^{16} \mathrm{H}_{2} / \mathrm{cm}^{2} \\
2.6 \times 1\end{array}$ \\
\hline
\end{tabular}

\subsection{Sticking Coefficients}

Knowledge of the molecular sticking coefficients $\sigma_{w}$ in Eqs. (13) and (14) is essential to understanding the dynamics of beam gas density. An apparatus has been assembled at BNL using photoelectron emission spectroscopy to measure sticking coefficients. ${ }^{42}$ Synchrotron light from a monochromator (photon energy $=170 \mathrm{eV}$ ) illuminates a surface, and the intensity and energy spectrum of photoelectrons are measured with a hemispherical electrostatic energy analyzer and channeltron detector. The sample is attached to a continuous-flow LHe cryostat and has a temperature of 4-5 K. Dosing gas is admitted to the vacuum chamber through a variable-temperature nozzle that can be directed at the sample surface. It is planned to vary the temperature of the nozzle from $294 \mathrm{~K}$ to $40 \mathrm{~K}$; the first measurements reported in Reference 38 are at $294 \mathrm{~K}$. The surface that has been investigated so far is a clean evaporated $50-100 \AA \mathrm{Cu}$ film; once this is understood, similar studies will be made with technical beam tube surfaces.

Figure 16(a) shows photoelectron energy spectra from the clean $4-5 \mathrm{~K} \mathrm{Cu}$ surface and after a dose of 7.4 Langmuirs (L) of $\mathrm{H}_{2}\left(1\right.$ Langmuir $=1 \times 10^{-6}$ Torr-s $=1.45 \times 10^{15} \mathrm{H}_{2} / \mathrm{cm}^{2}$ at $294 \mathrm{~K}$ ). The clean surface shows a sharp peak due to photoemission of $\mathrm{Cu} 3 \mathrm{~d}$ electrons and a flat, continuous, inelastic electron spectrum from the $\mathrm{Cu} 3 \mathrm{~d}$ peak to higher binding energies. The spectrum after exposure to $7.4 \mathrm{~L}$ of $\mathrm{H}_{2}$ shows an enhancement of the continuous spectrum below $-15 \mathrm{eV}$ due to inelastic scattering on hydrogen. The data in Figure 16(a) have been normalized to the same $\mathrm{Cu} 3 \mathrm{~d}$ intensity. The absolute intensity of the $\mathrm{Cu} 3 \mathrm{~d}$ peak actually decreases due to scattering in the adsorbed gas layer, and it is this decrease in intensity that is used as a measure of the hydrogen surface density. An example of this is shown in Figure 16(b); the electron energy analyzer has been tuned to the $\mathrm{Cu} 3 \mathrm{~d}$ feature, and the intensity plotted versus $\mathrm{H}_{2}$ dose. A linear decrease in intensity is observed at $0-2.3 \mathrm{~L}$; beyond $2.3 \mathrm{~L}$ the intensity is flat, indicating no further adsorption of $\mathrm{H}_{2}$. We interpret this as the adsorption of one monolayer of $\mathrm{H}_{2}$; the flattening occurs because the vacuum pressure is well below the saturation density of the $\mathrm{H}_{2}$ isotherm. An estimate of the sticking coefficient is readily obtained from a simple flux balance: $\sigma_{w} \times 2.3 \times 1.45 \times 10^{15}=s_{m}$, or $\sigma_{w}=s_{m} / 3.34 \times 10^{15} \sim 1$ for $294-\mathrm{K} \mathrm{H}_{2}$ on a $4-5-\mathrm{K}$ "clean" $\mathrm{Cu}$ 
surface. We don't actually know the monolayer $\mathrm{H}_{2}$ density for the evaporated $\mathrm{Cu}$ surface used for this experiment, but if it is similar to what we have measured for an electrodeposited $\mathrm{Cu}$ beam tube, then the sticking coefficient implied by the data in Figure 16(b) is close to unity. However, the enhancement of the $\mathrm{H}_{2}$ density over the substrate due to the formation of a gas jet by the doser has been neglected. In an identical experiment using $\mathrm{CO}$ (assumed to have unity sticking coefficient at $4 \mathrm{~K}$ ), the enhancement factor was found to be about 10 . This would reduce the estimated sticking coefficient for 294- $\mathrm{K} \mathrm{H}_{2}$ to approximately 0.1 , a value in reasonable agreement with sticking coefficients derived from $\mathrm{H}_{2}$ and $\mathrm{D}_{2}$ molecular beam experiments on LHe-cooled $\mathrm{Cu}(100)^{43}$ and the value 0.075 deduced from the $\mathrm{CDG}$ experiments.
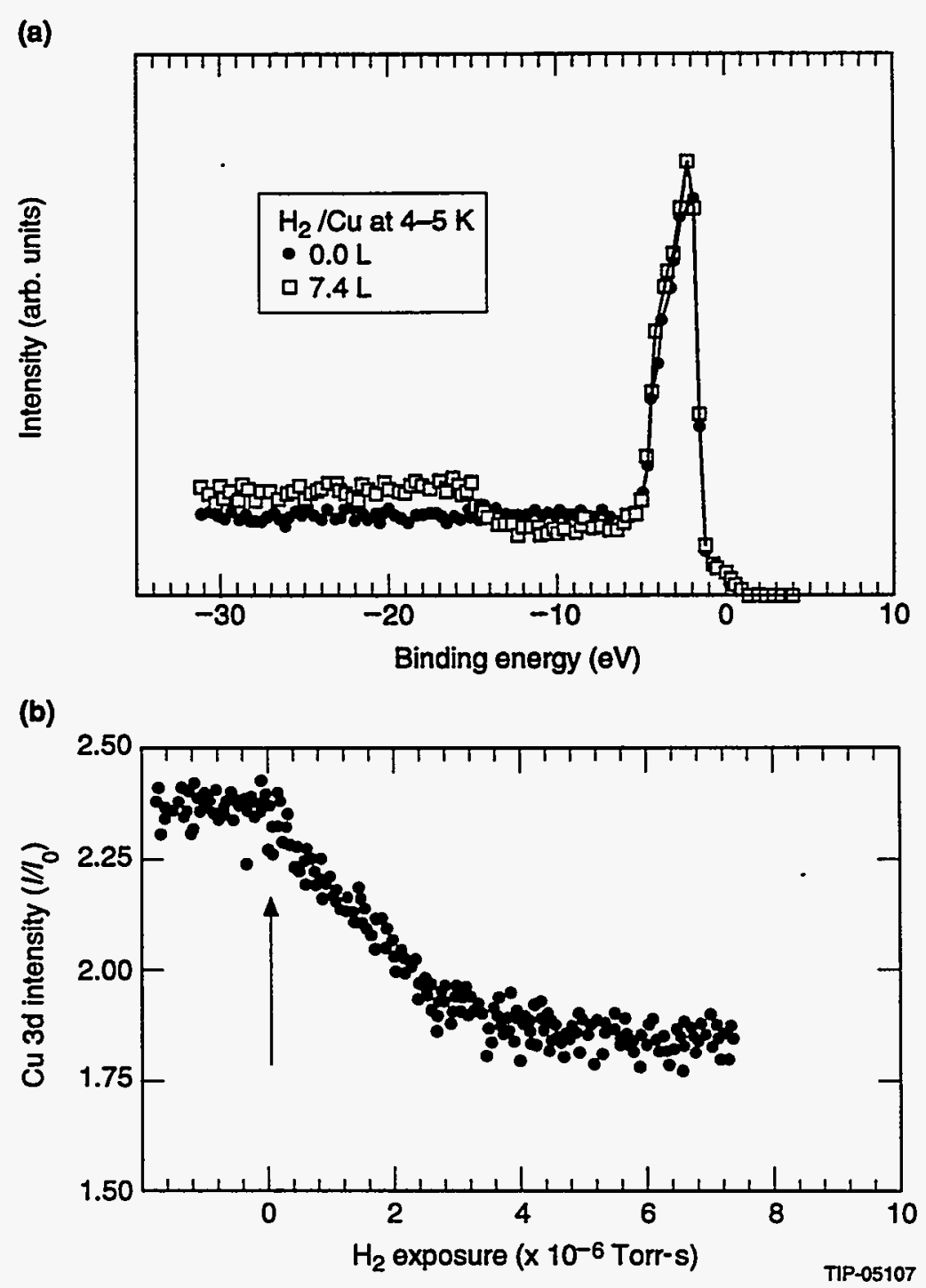

Figure 16. (a) Photoemission electron energy spectra on a 4-5 $\mathrm{K}$ clean $\mathrm{Cu}$ surface exposed to $\mathrm{H}_{2}$ dosing; (b) attenuation of the $\mathrm{Cu} 3 \mathrm{~d}$ photoelectron intensity versus $\mathrm{H}_{2}$ dose. ${ }^{38}$ 


\section{Liner Vacuum Conductance and Impedance}

The vacuum performance of a liner is defined by the conductance of the perforations. If the perforations are taken to be circular holes with diameter $d$ in a wall with thickness $t$, the conductance per unit length is given by

$$
C=\left(\frac{\pi}{16}\right) p N_{\mathrm{h}} d^{2} \bar{v},
$$

where $N_{\mathrm{h}}$ is the number of holes per unit length, $\bar{v}$ is the mean molecular speed, and $p=p(t / d)$ is the Clausing hole transmission probability. The holes increase the impedance seen by the beam compared to a smooth-wall beam tube and must be chosen to allow adequate safety margins for single-bunch instabilities. Below the cutoff frequency of the beam tube $(\sim 5 \mathrm{GHz})$ the transverse and longitudinal impedances of circular holes are given by ${ }^{44,45}$

$$
\begin{aligned}
& Z_{T, \text { holes }}=i g\left(\frac{4}{3 \pi}\right) \frac{Z_{0} R N_{\mathrm{h}} d^{3}}{D^{4}} \\
& \frac{Z_{L, \text { holes }}}{n}=\frac{D^{2}}{8 R} Z_{T, \text { holes }},
\end{aligned}
$$

where $D$ is the diameter of the liner, $2 \pi R$ is the liner circumference, $Z_{0}=377 \Omega$, and $g=g(t / d)$ is the thickness correction worked out by Gluckstern. ${ }^{45}$ These expressions for the impedance of holes are in good agreement with experimental measurements and computer simulation. ${ }^{46,47}$ The two thickness-dependent factors are plotted as functions of the ratio of thickness to hole diameter in Figure 17. The Clausing transmission factor decreases monotonically and rather slowly, while the impedance correction factor of Gluckstern initially decreases sharply with thickness and then reaches a thickness-independent asymptotic value $g=0.562$ for $t / d>0.625$. Since the hole impedance for constant conductance decreases linearly with the diameter $d$ of the holes, minimizing the impedance pushes one to the smallest possible diameter holes. Meanwhile, the liner must be thick enough to withstand the forces induced by a magnet quench; for electroplated $\mathrm{Cu}$ on the inside of a stainless steel tube, $t \approx 1 \mathrm{~mm}$ is required. ${ }^{48}$ Practically, a hole diameter must be chosen so the number of holes does not become excessive; going from $d / t=2$ to $d / t=1$ would require increasing the number of holes by a factor 5.2 to keep the conductance constant. Consequently, we provisionally choose $d / t=2, t=1 \mathrm{~mm}, d=2 \mathrm{~mm}, p(t / d=0.5)=0.67$, and $g(t / d=0.5)=0.56$ as working numbers.

An effective way of decreasing the hole impedance while holding the conductance constant is to use rounded-end longitudinal slots instead of circular holes. For thin, rounded-end slots Kurrenoy has derived the interesting result that the impedance approaches a constant value independent of the length of the slot and equal to 1.6 times the impedance of a circular hole with diameter equal to the width of the slot. ${ }^{49}$ In addition, the Clausing factor increases, requiring less hole area for a given pumping speed. Very long slots, however, have the disadvantage of radiating high-frequency, long-term wakefields, so a slot length-to-width ratio $\sim 3$ seems to be an optimal choice. The thickness correction analogous to the Gluckstern factor in Figure 17 
for circular holes has not been worked out for slots. However, Kurennoy states that it seems reasonable that the relative impedance ratio of thick slots to thick circular holes should be the same as for the thin case, with accuracy $10-20 \% .^{49}$

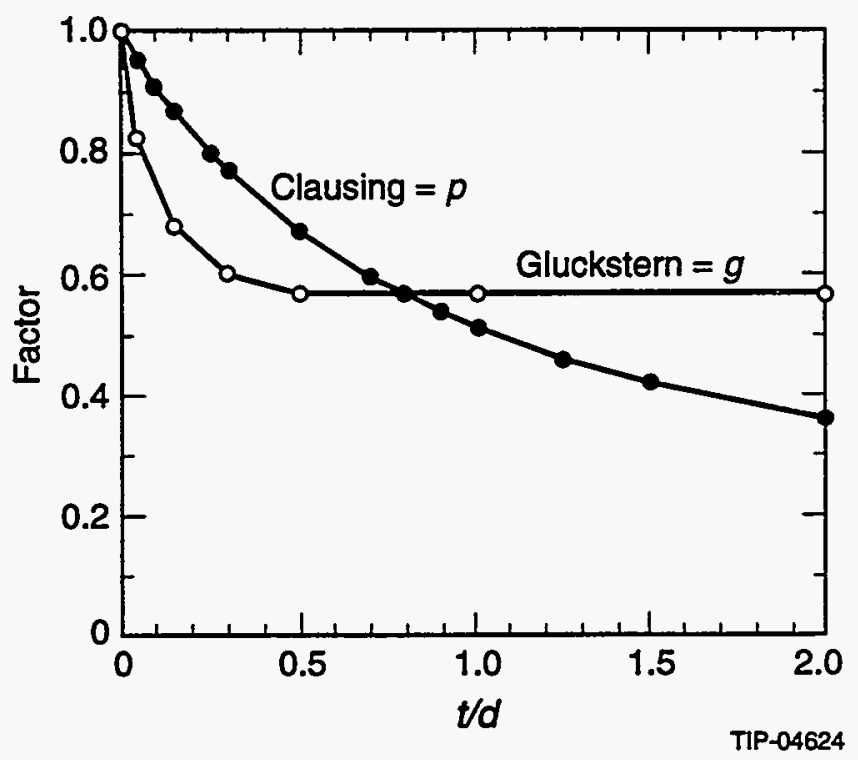

Figure 17. Clausing and Gluckstern hole-thickness correction factors versus the ratio of hole thickness to diameter.

In Figure 18 we have plotted the transverse and longitudinal impedances versus the fraction of wall area covered by holes in a liner. Cases with circular holes and rounded-end slots are shown, and the liner impedance has been added to the impedance of the rest of the accelerator beam-tube structures for the particular case of the SSC. The diameter of the circular holes and rounded slot ends has been taken to be $d=2 \mathrm{~mm}$, the inside diameter of the liner is $D=32 \mathrm{~mm}$, the liner circumference is $2 \pi R=70.4 \mathrm{~km}$, and the liner thickness has been taken to be $t=1 \mathrm{~mm}$. For the slot case, the slot length has been chosen to be $3 d=6 \mathrm{~m}$, and the impedance is reduced to $40 \%$ of the circular hole case, assuming the same hole area fraction. For the liner with circular holes (rounded slots) and a hole area fraction of 0.10 , the transverse impedance of holes is $24.5(9.8) \mathrm{M} \Omega / \mathrm{m}$, and the total transverse impedance has increased from $23.8 \mathrm{M} \Omega / \mathrm{m}$ for a beam tube of the same diameter without a liner to $48.3(33.6) \mathrm{M} \Omega / \mathrm{m}$, a factor of 2.0 (1.4). Furthermore, since the effective beam tube diameter would be larger without the liner, the real impedance increase is closer to a factor of 3.6 (2.5). These results favor the use of rounded slot-type holes and a hole area fraction no more than a few percent. Vacuum performance of such a liner is estimated in Section 6.

In addition to the low-frequency impedances below beam tube cutoff $(\sim 5 \mathrm{GHz})$, there have been concerns about TEM waves propagating in the annular region outside the liner, high-frequency wakefields and resonances occurring near or above the cutoff frequency, ${ }^{47}$ and trapped modes just below the cutoff frequency. Chou and Barts ${ }^{47}$ have studied the TEM mode with MAFIA and found no significant coupling between the inside and outside of a liner up to $30 \mathrm{GHz}$. They concluded that the impact of the coaxial structure on liner impedance was 
negligible. If a regular pattern of holes is adopted, resonances related to the hole spacing have been observed in computer simulations. Randomization of the hole spacing and size suppresses the resonances to negligible levels and is recommended to avoid high-frequency resonances and trapped modes for an actual accelerator. ${ }^{47,50}$ Walling recently extended liner impedance measurements to frequencies above the beam pipe cutoff and found reasonable agreement with the MAFIA calculations. ${ }^{51}$

(a)

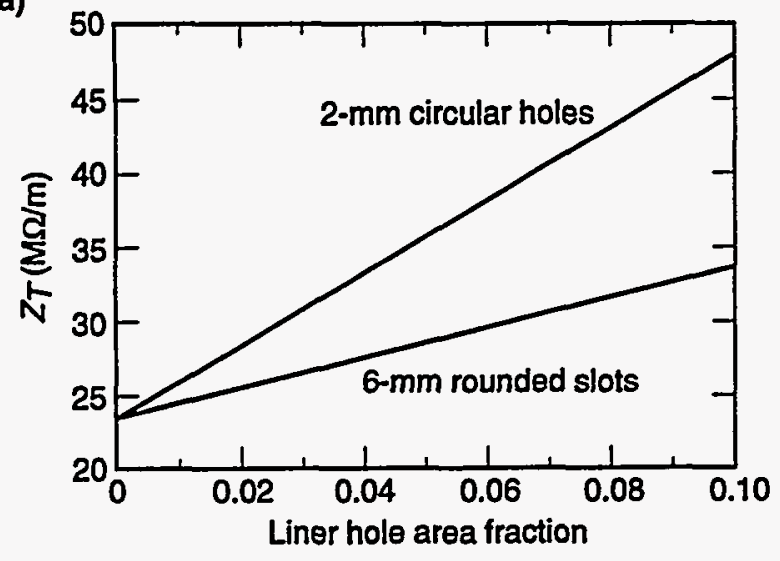

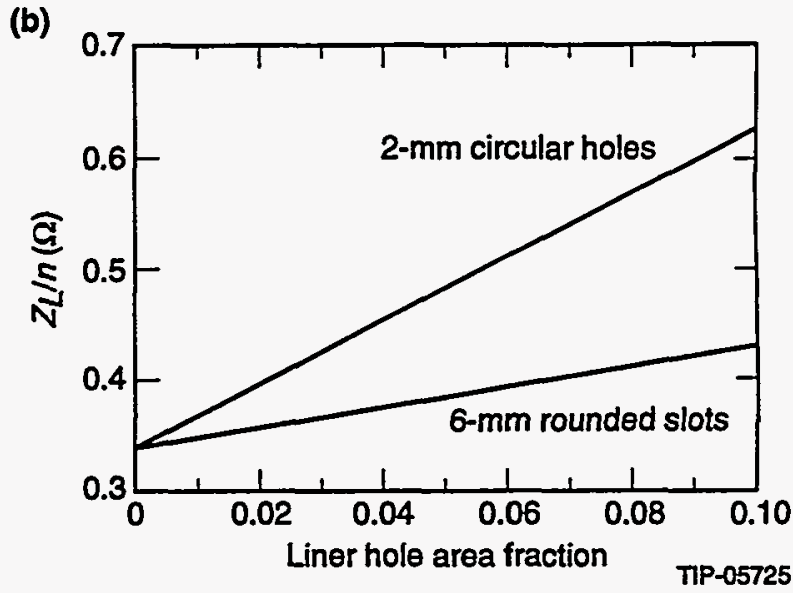

Figure 18. Transverse (a) and longitudinal (b) impedances versus the fraction of wall area covered by circular holes and rounded slots.

\section{Model Vacuum Calculations}

In this section we will present numerical solutions to the model equations in Section 2, using the experimental data described in Sections 3 and 4 to fix values of the various parameters. For the specific calculations we will generally assume the conditions representative of the SSC: $\dot{\Gamma}=1 \times 10^{16}$ photons $/ \mathrm{m} / \mathrm{s}$, photon critical energy $284 \mathrm{eV}$, beam tube radius $=1.6 \mathrm{~cm}$, and magnet cryostat temperature $=4.2 \mathrm{~K}$. Calculations are first presented for a simple beam tube and then for a liner configuration. Because of the absence of data on the photodesorption coefficients of physisorbed gases heavier than $\mathrm{H}_{2}$, the simple beam tube calculations are done only for $\mathrm{H}_{2}$. For the liner in steady state we need only the photodesorption coefficients of tightly bound molecules, and we will include $\mathrm{CO}$ because this data is available from the BINP experiments. Usually we will refer to the liner as being fabricated from stainless steel tubing with copper plating on the inside surface, since that is the case that has been most discussed and for which the most data exists. However, aluminum has also been discussed as a possibility and may have some fabrication advantages. ${ }^{5,53}$ 


\subsection{Simple Beam Tube}

Figure 19 shows a calculation of the $\mathrm{H}_{2}$ density versus integrated photon flux in a simple beam tube exposed to the synchrotron radiation intensity of the SSC. The solid line is for a tube of infinite length and represent a solution of Eq. (26). The three density components of the infinite-length solution are labeled (1) for the photodesorption of tightly bound hydrogen, (2) for the photodesorption of physisorbed $\mathrm{H}_{2}$, and (3) for the $\mathrm{H}_{2}$ isotherm. The uppermost solid curve is the sum of the three components and isn't labeled. The integrated photon flux shown in Figure 19 extends from $10^{19}$ photons $/ \mathrm{m}$ to $10^{22}$ photons $/ \mathrm{m}$, corresponding to $16.7 \mathrm{~min}$ to 11.6 days of operation at the SSC design intensity of $10^{16}$ photons $/ \mathrm{m} / \mathrm{s}$. For the photodesorption of tightly bound hydrogen we have assumed photodesorption coefficients $\eta_{1}=\eta_{2}=$ constant $=2 \times 10^{-3}$ for simplicity of illustration. These correspond to the initial magnitude of $4.2 \mathrm{~K}$ photodesorption coefficient in Figure 10. The calculation began at $\Gamma=0$ photons $/ \mathrm{m}$, with the surface density of physisorbed $\mathrm{H}_{2}$ set equal to zero. In this simplified calculation with constant $\eta_{1}+\eta_{2}$, the surface density of physisorbed $\mathrm{H}_{2}$ (Eq. (27)) and the photodesorption coefficient $\eta^{\prime}$ will increase linearly with the integrated photon flux. The question arises what to use for the product of sticking coefficient, $\sigma_{w}$, and ideal wall pumping speed, $S_{w}=A_{w} \frac{\bar{v}}{4}$, in the denominators of Eq. (26). For the sticking coefficient of photodesorbed molecules we have taken $\sigma_{w}=0.075$, which is consistent with the CDG experiment ${ }^{16}$ and the photoelectron emission spectroscopy measurements at BNL. ${ }^{42}$ Analysis of the CDG experiment gave a value $\bar{v}=1.8 \times 10^{5} \mathrm{~cm} / \mathrm{s}$ for the mean speed of photodesorbed molecules. Since this report has been in preparation the new $\mathrm{H}^{+} / \mathrm{H}^{-}$ion beam experiment at BINP has obtained a somewhat lower value, $\bar{v}=(8 \pm 1.5) \times 10^{4} \mathrm{~cm} / \mathrm{s}^{40}$ This experiment is described briefly in Section 7.1; since it gives a more conservative estimate of the vacuum conditions we will use it in the calculations. We do not make a distinction between the product of sticking coefficient and speed for the desorption of tightly bound and physisorbed molecules for the simple reason that there is no experimental information available. It will emerge from the calculation that except for a very brief period of the order of a few minutes following initial exposure to photons, the density is dominated by photodesorption of physisorbed $\mathrm{H}_{2}$ until a surface density $s \approx s_{m}=3 \times 10^{15} / \mathrm{cm}^{2}$ is reached and thermal desorption takes over. For this reason it seems likely that the CDG and BINP data for sticking coefficient and mean molecular speed apply to photodesorption of physisorbed $\mathrm{H}_{2}$. For the photodesorption coefficient of physisorbed $\mathrm{H}_{2}$ we take $\eta_{0}^{\prime}=0.525$ and $\sigma_{w}=0.075$, obtained by combining the measurement $\frac{\eta_{0}^{\prime}}{\sigma_{w}}=7.0$ from the BINP data in Figure 9 with the CDG estimate of the sticking coefficient. Finally, for the isotherm density component we take the BET Eq. (15) with the parameters that are given following the discussion of the BINP data in Figure 12: $\alpha=1.3 \times 10^{4}$, monolayer density $s_{m}=3.0 \times 10^{15} \mathrm{H}_{2} / \mathrm{cm}^{2}$, and saturation density $n_{\text {sat }}(4.2 \mathrm{~K})=2.0 \times 10^{12} \mathrm{H}_{2} / \mathrm{cm}^{3}$. From $\Gamma=1.0 \times 10^{19}$ to $7.2 \times 10^{20}$ photons $/ \mathrm{m}$, the $\mathrm{H}_{2}$ density is dominated by the photodesorption of physisorbed $\mathrm{H}_{2}$. Near $\Gamma=7.2 \times 10^{20}$ photons $/ \mathrm{m}$ the surface density of physisorbed $\mathrm{H}_{2}$ approaches a monolayer, and the $\mathrm{H}_{2}$ isotherm density becomes dominant as it rapidly increases over three orders of magnitude. Although the photodesorption of 
tightly bound hydrogen seems to be playing a relatively minor role, it determines the magnitude of integrated flux that is requried to accumulate a monolayer.

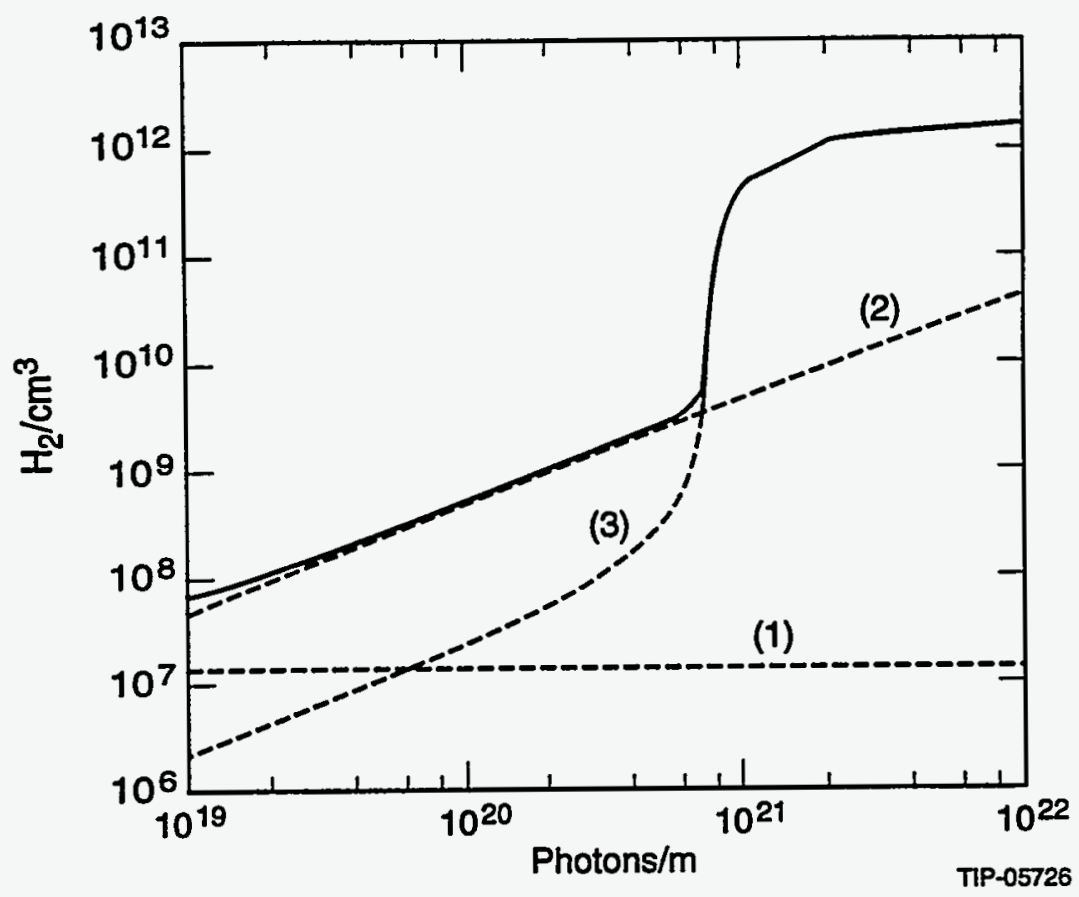

Figure 19. $\mathrm{H}_{2}$ density versus integrated photon flux for an infinitely long beam tube at $4.2 \mathrm{~K} ; \mathrm{\Gamma}=1 \times 10^{19}$ $1 \times 10^{22}$ photons $/ \mathrm{m}$. The individual components shown by the dashed lines are labeled (1) for photodesorption of tightly bound hydrogen, (2) for photodesorption of physisorbed molecules, and (3) for thermal desorption of physisorbed molecules. The solid line is the sum of the components.

Even though the portion of the calculation presented in Figure 19 begins at the relatively low integrated photon flux of $1 \times 10^{19}$ photons $/ \mathrm{m}$, the $\mathrm{H}_{2}$ density is already dominated by the photodesorption of physisorbed $\mathrm{H}_{2}$, which has reached $s=4 \times 10^{13} \mathrm{H}_{2} / \mathrm{cm}^{2}$, or $1.3 \%$ of a monolayer. Since the calculation was started off with zero physisorbed $\mathrm{H}_{2}$, as in an experiment that has just been cooled to $4.2 \mathrm{~K}$ before exposure to photons, it is clear that if one looks at low enough photon exposure there will be a region where the density is dominated by the photodesorption of tightly bound hydrogen. This region is shown in Figure 20, where the density of the infinite-length solution and the three components are plotted from $\Gamma=10^{17}$ to $10^{20}$ photons $/ \mathrm{m}$. Before $\Gamma=2.9 \times 10^{18}$ photons $/ \mathrm{m}$, the dominant density component is due to the photodesorption of tightly bound hydrogen. The photon flux $2.9 \times 10^{18}$ photons $/ \mathrm{m}$ at which photodesorption of physisorbed $\mathrm{H}_{2}$ takes over as the dominant component is determined by $\eta^{\prime}=\eta_{1}$, or for constant $\eta_{1}$ and $\eta_{2}$ as we have assumed here, $\Gamma=\frac{\eta_{1}}{\eta_{1}+\eta_{2}} \frac{A_{w} s_{m}}{\eta_{0}^{\prime}}$. The $\mathrm{H}_{2}$ surface density at this point has reached $s=1.1 \times 10^{13} / \mathrm{cm}^{2}$, or $0.4 \%$ of a monolayer, and at $10^{16}$ photons $/ \mathrm{m} / \mathrm{s}$ the elapsed time from the beginning of photon exposure is only $287 \mathrm{~s}$, or approximately $5 \mathrm{~min}$. 


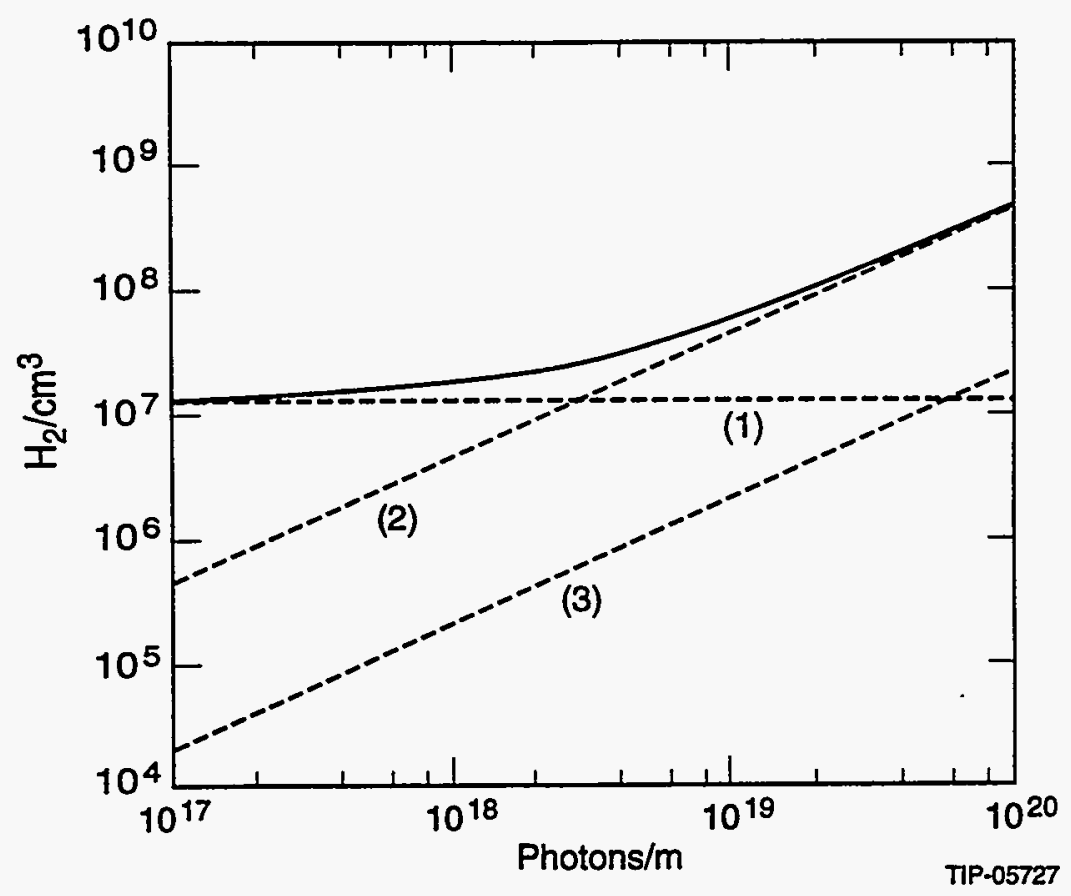

Figure 20. $\mathrm{H}_{2}$ density versus integrated photon flux for an infinitely-long beam tube at $4.2 \mathrm{~K} ; \Gamma=1 \times 10^{17}$ $1 \times 10^{20}$ photons $/ \mathrm{m}$.

For the calculations in Figures 19 and 20 we have assumed $\eta_{1}=\eta_{2}$, whereas what we know from the experiments is the sum $\eta_{1}+\eta_{2}$, and we don't know how the photodesorption of tightly bound hydrogen divides up between that which appears directly in the gas phase as $\mathrm{H}_{2}\left(\eta_{1}\right)$ and that which appears first as physisorbed $\mathrm{H}_{2}$ on the surface $\left(\eta_{2}\right)$. Here we comment on what happens to the calculations in Figures 19 and 20 if we allow $\eta_{1}$ and $\eta_{2}$ to vary while holding the sum $\eta_{1}+\eta_{2}$ constant. Under these circumstances the surface density of physisorbed $\mathrm{H}_{2}$ and the magnitude of $\eta^{\prime}$ versus $\Gamma$ are unchanged since they depend on $\eta_{1}+\eta_{2}$. It follows that the density due to desorption of physisorbed $\mathrm{H}_{2}$ and the $\mathrm{H}_{2}$ isotherm density versus $\Gamma$ are also unchanged. All that changes is the magnitude of the density due to photodesorption of tightly bound hydrogen and its contribution to the total $\mathrm{H}_{2}$ density (component 1 in Figures 19 and 20). With $\eta_{1}+\eta_{2}=$ constant, as $\eta_{1}$ goes to zero this density component vanishes; as $\eta_{2}$ goes to zero it doubles compared to the assumption $\eta_{1}=\eta_{2}$. This uncertainty will only make a significant difference to the plot of $\mathrm{H}_{2}$ density versus $\Gamma$ for $\Gamma<10^{19}$ photons $/ \mathrm{m}$. This suggests that a way to get an experimental measurement of $\eta_{1}$ and $\eta_{2}$ separately is by carefully measuring the $\mathrm{H}_{2}$ density versus photon flux for $0<\Gamma<10^{19}$ photons/m after initial cooldown. The density versus photon flux in this range is given by

$$
n=\frac{\eta_{1} \dot{\Gamma}}{\sigma_{w} S_{w}}+\frac{\eta_{0}^{\prime} \dot{\Gamma}}{\sigma_{w} S_{w}} \frac{\eta_{1}+\eta_{2}}{A_{w} s_{m}} \Gamma
$$

The constant part of the density versus $\Gamma$ would give $\eta_{1}$; the linearly increasing part of the density would give $\eta_{1}+\eta_{2}$. 
The reader may have noticed that for the calculations in Figures 19 and 20 we have used the mean speeds of the individual components in Eq. (26) rather than a density-weighted average everywhere, as described in Section 2 . The total density would be the same in either case, but when the individual component speeds are used, each component is direct reading in density. This distinction is described further in Appendix A; the components of Eq. (A5) rather than Eq. (A6) have been plotted.

The calculations in Figures 19 and 20 are for an infinitely long beam tube. For finitelength beam tubes the density at the center of the tube will respond to the boundary conditions at the ends when the axial diffusion time given by Eq. (30) has been reached. The density at the center of the tube then reverts to Eq. (18) at $z=0$, and the full axial solution is a parabola. Prior to this time the solution is a truncated parabola. Assuming $n( \pm L / 2) \leq n(0)$, the surface density of physisorbed $\mathrm{H}_{2}$ ceases to increase because of pumping out the ends; in fact, it would decrease if the surface began to clean up and $\eta_{1}+\eta_{2}$ decreased with increasing $\Gamma$. In our simplified calculation with $\eta_{1}+\eta_{2}=$ constant, the surface density and volume density of $\mathrm{H}_{2}$ would simply stay constant with continued photon exposure. For the molecular speed appearing in the diffusion coefficient $D$, a density-weighted average over the three gas components is to be used. For the photodesorbed molecules we use $\bar{v}=8 \times 10^{4} \mathrm{~cm} / \mathrm{s}$, and for thermally desorbed molecules $\bar{v}=2.1 \times 10^{4} \mathrm{~cm} / \mathrm{s}$. We have taken the density components of the infinitely-long calculation in Figures 19 and 20 to calculate the mean speed and density at the centers of tubes with lengths $=1 \mathrm{~m}, 5 \mathrm{~m}, 15 \mathrm{~m}$, and $90 \mathrm{~m}$. The results are shown in Figure 21, together with the infinitely-long solution superimposed. The transitions from infinitely-long to finite-length solutions occur at the intersections. For $L=1 \mathrm{~m}$ the transition flux is $\Gamma=1.5 \times 10^{20}$ photons $/ \mathrm{m}$, and diffusion to the ends prevents it from reaching the $\mathrm{H}_{2}$ isotherm-dominated region. The density at transition is $7.6 \times 10^{8} / \mathrm{cm}^{3}$. For this case the dominant axial diffusion process is photodesorption of physisorbed $\mathrm{H}_{2}$. The step increase in density in the finite-length solutions near $8 \times 10^{20}$ photons/m occurs when the density of the $\mathrm{H}_{2}$ isotherm dominates the density components due to photodesorption. For the $5-\mathrm{m}$ and $15-\mathrm{m}$ cases the transition fluxes are $\Gamma=7.7 \times 10^{20}$ and $1.1 \times 10^{21}$ photons $/ \mathrm{m}$, and they have reached the $\mathrm{H}_{2}$ isotherm-dominated region with densities $n=6 \times 10^{10}$ and $6.1 \times 10^{11} / \mathrm{cm}^{3}$, respectively. For these cases the dominant axial diffusion process is thermal desorption of physisorbed $\mathrm{H}_{2}$. The finite-length, $90-\mathrm{m}$ solution lies well above the infinitely-long solution for the range of integrated photon flux shown in Figure 21. The solution in this case therefore remains on the infinitely-long branch for the duration of the calculation.

It should perhaps be pointed out that the finite-length solutions in Figure 21 are not plots of density versus integrated photon flux in the sense of Figure 19. At each value of the integrated photon flux they are simply the finite-length densities that would pertain if they were in fact the solutions. The only solutions that actually pertain are at the intersections with the infinite-length solution. Once these are reached the density is clamped at that point and ceases to evolve, since we have assumed $\eta_{1}+\eta_{2}$ is constant. If $\eta_{1}+\eta_{2}$ is not constant then putting in the actual dependence of $\eta_{1}+\eta_{2}$ on $\Gamma$ would determine the evolution of density from the intersection point forward. Since the finite-length solution given by Eq. (18) depends only upon $\eta_{1}+\eta_{2}$ and not $\eta^{\prime}$ or the isotherm, one might expect that the gas density, including that which is diffusing out the ends, is due to direct desorption of tightly bound gas. This would be true if the surface were not cryosorbing. However, as we have just seen by the examples in Figures 19-21, 
this gas density tends to be dominated by photodesorption or thermal desorption of physisorbed molecules in cases where the temperature of the beam tube is low enough to be cryosorbing. The density due to desorption of tightly bound hydrogen is suppressed by cryosorption on the surface. Meanwhile, photodesorption and thermal desorption of physisorbed molecules have increased the density precisely to that which would pertain with no physisorption. These processes do this by recycling physisorbed molecules from the surface, but they do not increase the total inventory of molecules in the gas phase plus physisorbed on the surface, and diffusing out the ends. New molecules are created only by photodesorption of the tightly bound $\mathrm{H}, \mathrm{C}$, and $\mathrm{O}$. It is that creation rate, depending on $\eta_{1}+\eta_{2}$, divided by the axial conductance that appears in the finitelength, quasi-steady state solution whether physisorption is present or not. If the $\eta_{1}+\eta_{2}$ process were somehow suddenly turned off but photodesorption and thermal desorption of physisorbed molecules continued, the quasi-steady state assumption $A_{w} \frac{\partial s}{\partial t}=0$ would be violated until all the molecules had been pumped out the beam tube and the steady state solution $n=0, s=0$ were reached, which is consistent with Eqs. (18) and (19).

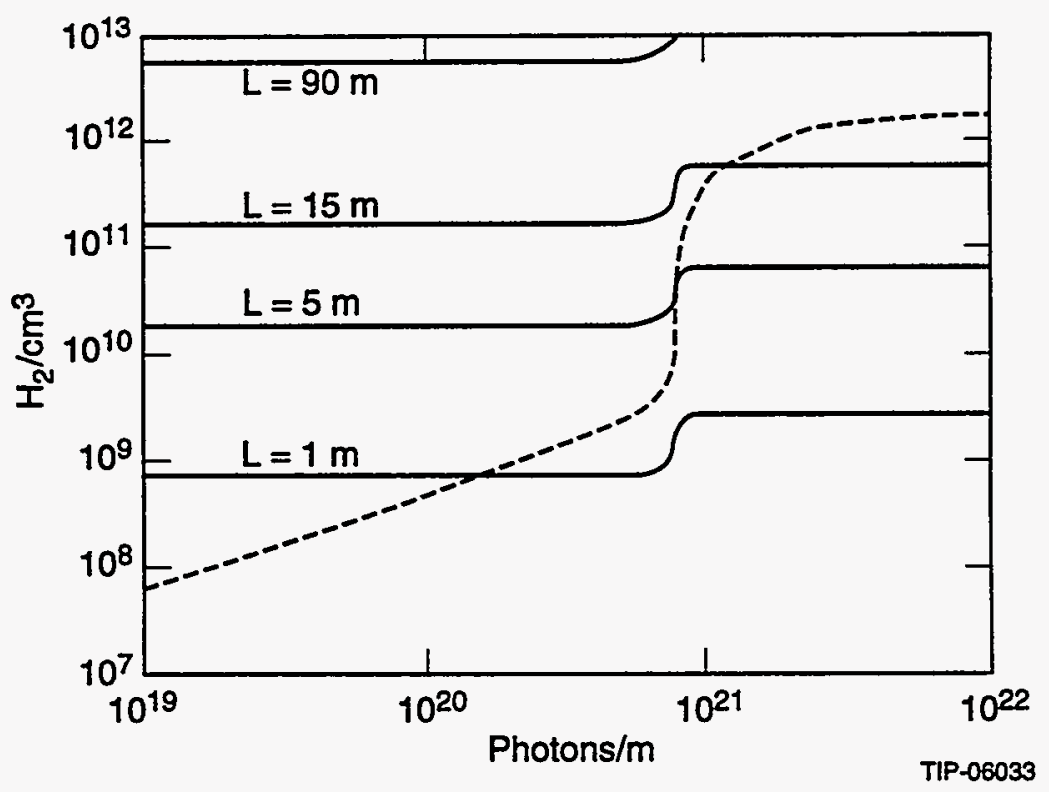

Figure 21. Intersection of finite-length beam tube solutions with the infinite-length solution at $4.2 \mathrm{~K} ; L=1 \mathrm{~m}$, $5 \mathrm{~m}, 15 \mathrm{~m}$, and $90 \mathrm{~m}$, with boundary condition $n( \pm L / 2)=0$.

The calculations in Figures 19-21 have been done with a photon flux of $10^{16}$ photons $/ \mathrm{m} / \mathrm{s}$. If the photon intensity is changed, the magnitudes of the density components due to photodesorption of tightly bound and physisorbed hydrogen will scale linearly with $\dot{\Gamma}$, and the component due to the isotherm or thermal desorption will be unchanged, all versus $\Gamma$. However, if the density is plotted versus time, the magnitude of the tightly bound component will increase linearly with $\dot{\Gamma}$, and the magnitude of the physisorbed component will increase as $\dot{\Gamma}^{2}$. The isotherm component would initially increase linearly with $\dot{\Gamma}$ for $x \ll 1$ and $\alpha y \ll 1$. As one monolayer is approached, the scaling becomes more complicated and can be deduced by inverting Eq. (15). 
In Figure 22 we repeat the calculation in Figure 21 with finite density at the tube ends $n(L / 2)=6 \times 10^{9} \mathrm{H}_{2} / \mathrm{cm}^{3}$. The infinite-length solution is unchanged, whereas the finite-length tubes all have a constant $6 \times 10^{9} / \mathrm{cm}^{3}$ added to them. This has the effect of pushing the transitions from the infinitely-long solution to the finite-length solutions to larger values of integrated photon flux, since axial diffusion out of the tubes is now being partially compensated by axial diffusion into the tubes. The change is most noticeable for the shortest length tube, and the change in transition flux becomes less noticeable as the rapidly rising portion of the isotherm is reached (e.g., the 5-m and 15-m solutions). As the density at the ends is increased the shortest tubes will eventually reach the rapidly rising portion of the isotherm before making a transition to the finite-length solutions. In this way the $\mathrm{H}_{2}$ isotherm was observable even with 1 -m-long tubes in the BINP experiments. ${ }^{37}$

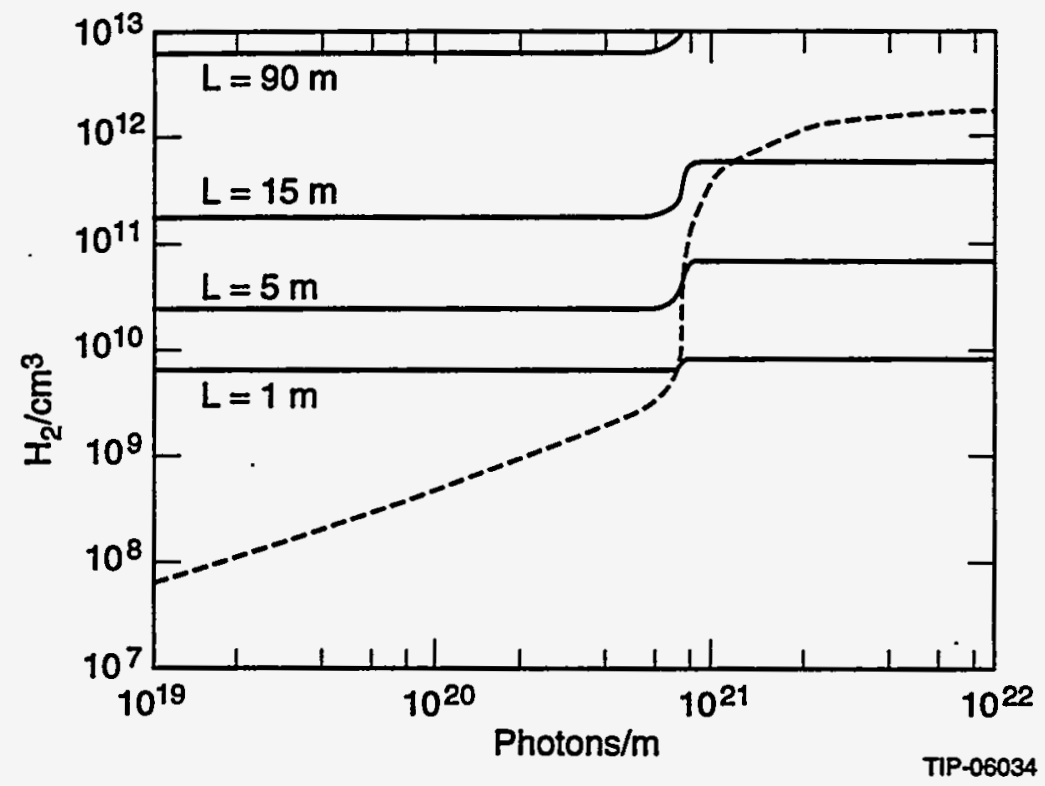

Figure 22. Intersection of finite-length beam tube solutions with the infinite-length solution at $4.2 \mathrm{~K} ; L=1 \mathrm{~m}$, $5 \mathrm{~m}, 15 \mathrm{~m}$, and $90 \mathrm{~m}$, with boundary condition $n( \pm L / 2)=6 \times 10^{9} \mathrm{H}_{2} / \mathrm{cm}^{3}$.

The calculations in Figures 21 and 22 indicate the difficulty of achieving the required vacuum conditions in a superconducting proton collider with distributed pumping. Keeping the $\mathrm{H}_{2}$ density below $3 \times 10^{8} / \mathrm{cm}^{3}$ would require placing even ideal pumps at less than 1 -m intervals (e.g., the 1-m solution in Figure 21).

The primary beam tube temperature effect in these calculations is the $\mathrm{H}_{2}$ isotherm. In Figures 23 and 24 we have redone the calculations of Figures 19 and 21 with the beam tube temperature reduced from $4.2 \mathrm{~K}$ to $3.0 \mathrm{~K}$. The effect is dramatic: the $\mathrm{H}_{2}$ saturation density is reduced almost four orders of magnitude, from $2.0 \times 10^{12} / \mathrm{cm}^{3}$ to $3.1 \times 10^{8} / \mathrm{cm}^{3}$. If the $\mathrm{H}_{2}$ isotherm density were the only problem, then this would practically get one into a range of acceptable vacuum for a superconducting proton collider. However, one sees from Figures 23 and 24 that the density due to photodesorption of physisorbed $\mathrm{H}_{2}$ gets into an unacceptable range rather quickly, and so far no account has been taken of the possible photodesorption of physisorbed gases heavier than $\mathrm{H}_{2}$. 


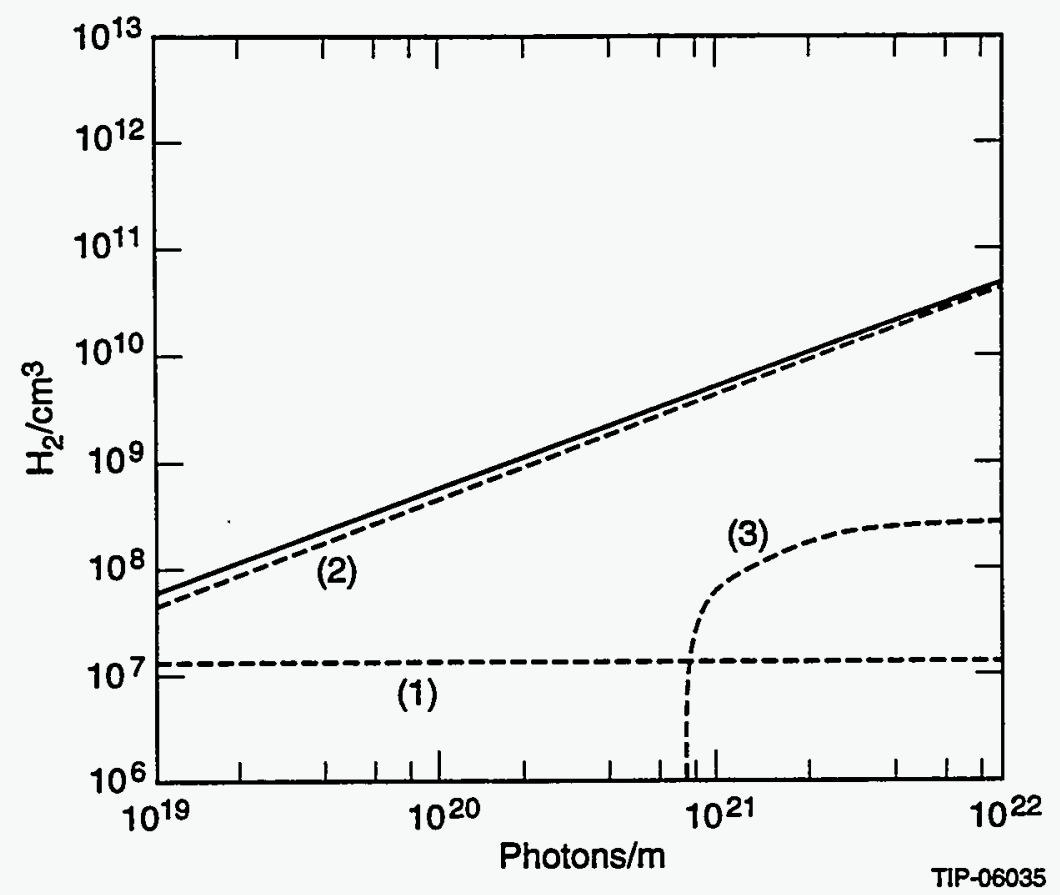

Figure 23. $\mathrm{H}_{2}$ density versus integrated photon flux for an infinitely-long beam tube at $3.0 \mathrm{~K} ; \Gamma=1 \times 10^{19}$ $1 \times 10^{22}$ photons/m.

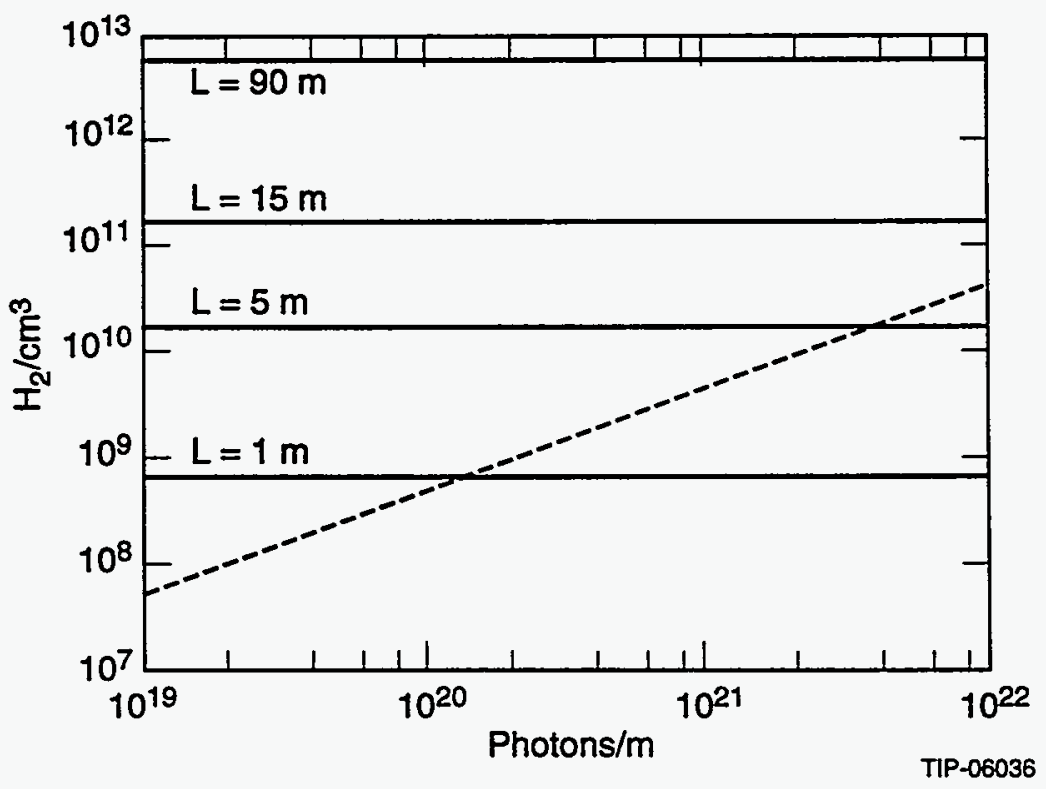

Figure 24. Intersection of finite-length beam tube solutions with the infinite-length solution at $3.0 \mathrm{~K} ; L=1 \mathrm{~m}$, $5 \mathrm{~m}, 15 \mathrm{~m}$, and $90 \mathrm{~m}$, with boundary condition $n( \pm L / 2)=0$. 


\subsection{Liner}

The solution for the $\mathrm{H}_{2}$ density in a liner given by Eq. (37) is shown in Figure 25 with the infinite-length, 4.2-K simple beam tube solution superimposed. The physical conditions are the same as for the calculations in the previous section. The linear has $3002-\mathrm{mm}$ holes $/ \mathrm{m}$ with hole thickness $1 \mathrm{~mm}$ and transmission probability $p=0.67$. The $\mathrm{H}_{2}$ density, $3.3 \times 10^{8} / \mathrm{cm}^{3}$, is roughly half the density of the 1-m-length solution, with strong pumping at the ends in Figure 21. The treatment of the molecular speed has been done in exactly the same way as for the finite-length simple beam tubes. Because the assumed molecular speed of the photodesorbed molecules, $\bar{v}=8 \times 10^{4} \mathrm{~cm} / \mathrm{s}$, corresponds to an effective temperature $\sim 60 \mathrm{~K}$, the calculations are not sensitive to the actual temperature of the liner until it reaches $60 \mathrm{~K}$.

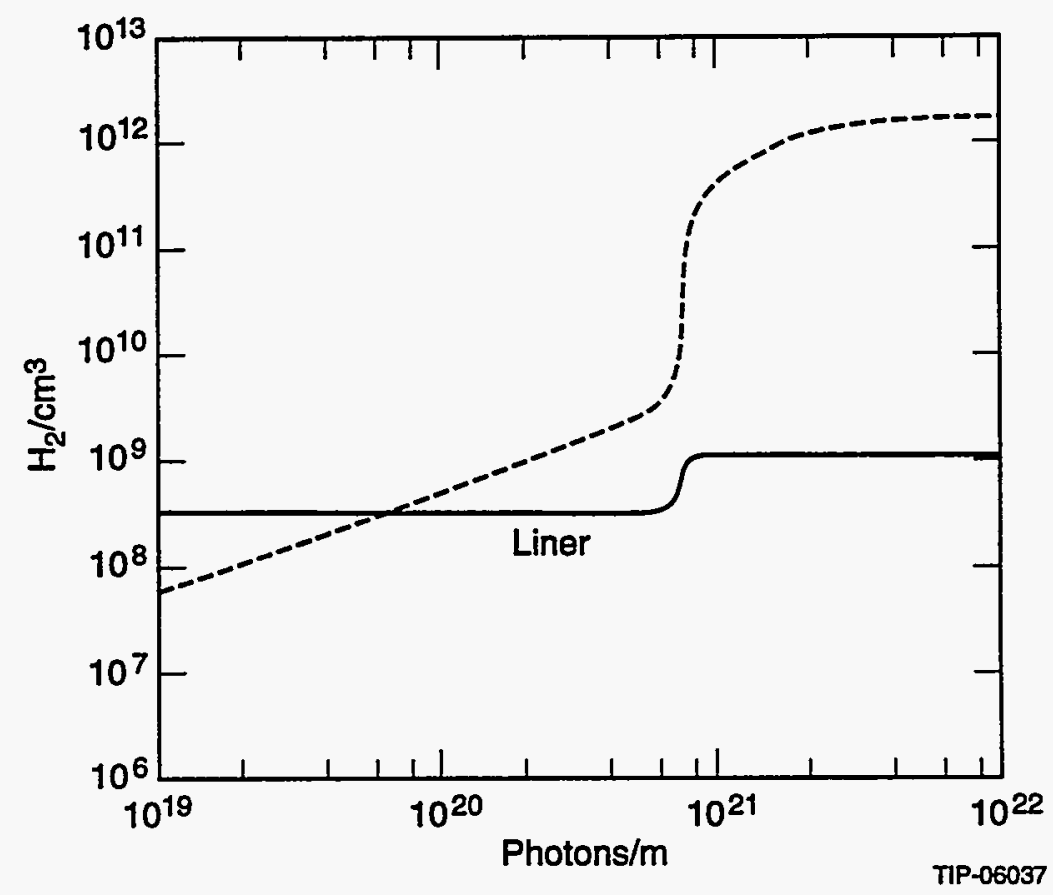

Figure 25. Intersection of a liner solution with the infinite-length solution at $4.2 \mathrm{~K}$.

Although the magnitude of the quasi-steady state liner density does not depend on whether the liner surface is cryosorbing, the details of what is going are quite different in these two cases. If we assume the liner is at low enough temperature to be cryosorbing, then there will be an initial period of time when the surface of the liner has not yet reached the steady state solution and the surface is actively pumping. During this initial period the $\mathrm{H}_{2}$ density will fall on the infinite-length beam tube solution and the surface density of physisorbed $\mathrm{H}_{2}$ on the liner will increase with integrated photon flux. Eventually the $\mathrm{H}_{2}$ density in the beam tube will increase due to photodesorption or thermal desorption of the physisorbed $\mathrm{H}_{2}$, and the 
solution will intersect and go over to the liner solution. Although the form of the solution given by Eq. (37) depends only on $\eta_{1}+\eta_{2}$, and therefore suggests that the gas being pumped is due to direct photodesorption of tightly bound $\mathrm{H}_{2}$, it is actually the photodesorption of physisorbed $\mathrm{H}_{2}$ that is dominating the denisty in the beam tube. The story is essentially the same as the one for pumping at the ends of finite-length beam tubes. If the liner is at $4.2 \mathrm{~K}$, the intersection can be seen in Figure 25 and occurs at $\Gamma=6.5 \times 10^{19}$ photons $/ \mathrm{m}$, or $1.8 \mathrm{~h}$ for the assumed intensity of $10^{16}$ photons $/ \mathrm{m} / \mathrm{s}$. At this time the surface density of $\mathrm{H}_{2}$ on the liner has reached $2.7 \times 10^{14} / \mathrm{cm}^{2}$ or $9.1 \%$ of a monolayer. Beyond $\Gamma=6.5 \times 10^{19}$ photons $/ \mathrm{m}$ the liner solution would stay clamped at $3.3 \times 10^{8} / \mathrm{cm}^{3}$ until the $\mathrm{H}_{2}$ pumping capacity of the region outside the liner were reached, which is not considered in Figure 25. If the magnet cryostat bore tube outside the liner is at $1.9 \mathrm{~K}$ as in the LHC, the saturated vapor pressure of $\mathrm{H}_{2}$ is extraordinarily low and the liner would pump indefinitely. If the outer bore tube is at $4.2 \mathrm{~K}$, then as one monolayer is approached the vapor pressure of adsorbed $\mathrm{H}_{2}$ will rise to equal that inside the liner and the liner will cease to pump. The solution will then revert to one of the simple beam tube solutions pumped from the ends. This can be dealyed by adding high-porosity cryopumping material to the surface of the bore tube. Charcoal is one possibility. Recently Rao and coworkers at CEBAF have obtained very high pumping capacities for a layer of anodized aluminum at $4.3 \mathrm{~K}$, and this may be attractive for accelerator beam tube applications. ${ }^{54}$

If the liner is at high enough temperature that it does not adsorb $\mathrm{H}_{2}$, then of course the molecules being pumped directly by the liner will be the photodesorbed, tightly bound hydrogen. Compared to the cryosorbing case, the density due to desorption of tightly bound hydrogen will increase by the ratio of the wall pumping speed to the hole conductance, while the other components will vanish. The liner solution in this case will reach the quasi-steady state solution essentially instantaneously with time constant $\tau=\frac{4 \pi a^{2}}{p N_{h} A_{h} \bar{v}} \sim 10 \mathrm{~ms}$.

In order to estimate the actual performance of a liner it is necessary to include the photodesorption of the gases heavier than $\mathrm{H}_{2}$ when calculating the vacuum-limited luminosity lifetime and beam-gas scattered power according to Eqs. (8) and (9). For these purposes it can be convenient to combine the liner solution, Eq. (37), for the density of each component with Eqs. (8) and (9) to define $\mathrm{H}_{2}$ equivalent densities and photodesorption coefficients:

$$
n_{\mathrm{H}_{2} \mathrm{eq}}=\sum_{j} \frac{\sigma_{p, j}}{\sigma_{p, \mathrm{H}_{2}}} n_{j}
$$

and

$$
\eta_{\mathrm{H}_{2} \mathrm{eq}}=\sum_{j} \frac{\sigma_{p, j}}{\sigma_{p, \mathrm{H}_{2}}} \frac{C_{\mathrm{H}_{2}}}{C_{j}} \eta_{j}
$$

The factors weighting the densities and desorption coefficients for gases heavier than $\mathrm{H}_{2}$ are sizable. From Table 2 the density weights for the SSC case are 1.0, 6.04, 9.23, and 14.4 for $\mathrm{H}_{2}$, $\mathrm{CH}_{4}, \mathrm{CO}$, and $\mathrm{CO}_{2}$. If the photodesorbed molecules of each species are assumed to have the same mean energy, then the photodesorption coefficient weights are increased by an additional factor equal to the square root of the mass ratio relative to $\mathrm{H}_{2}$, becoming $1.0,17.1,34.5$, and 67.6. We have used the 4.2-K desorption coefficients in Figure 10 to calculate the vacuum-limited luminosity lifetime and scattered beam power due to photodesorption of $\mathrm{H}_{2}$ and $\mathrm{CO}$. The results 
are shown in Figure 26 for the SSC case with $10^{16}$ photons $/ \mathrm{m} / \mathrm{s}$ and the liner parameters that were considered for Figure 25. The results are clearly dominated by CO. Photodesorption data is not yet available for $\mathrm{CO}_{2}$ at $4.2 \mathrm{~K}$, but we may expect from room-temperature data that $\mathrm{CO}_{2}$ will have an effect about equal to $\mathrm{CO}$ and will lead to a further reduction of luminosity lifetime and an increase in scattered beam power by roughly a factor of two. For the SSC the goal for the vacuum-limited luminosity lifetime was $150 \mathrm{~h}$ at design intensity. We see from Figure 26(a) that the lifetime at design intensity is never worse than $80 \mathrm{~h}$, and the goal would be met after conditioning with approximately $7 \times 10^{21}$ photons $/ \mathrm{m}$ if $\mathrm{H}_{2}$ and $\mathrm{CO}$ were the only important gases. This corresponds to about 8 days' operation at design intensity or, what is more likely, 80 days at $10 \%$ design intensity during the initial conditioning of the accelerator. At $10 \%$ design intensity the luminosity lifetime would be hundreds of hours and not an operational concern. In Figure 26(b) we see that the maximum scattered beam power on $\mathrm{H}_{2}$ and $\mathrm{CO}$ at design intensity is always less than $10 \mathrm{~mW} / \mathrm{m}$ and less than $10 \%$ of the synchrotron radiation power. This would not be a serious cryogenic concern either locally or for the entire accelerator. Even supposing that the accelerator has been conditioned and has achieved design intensity, component replacement with unconditioned beam tube would permit immediate return to operation at full intensity without danger of exceeding the local bound on beam scattered power, $\sim 0.6 \mathrm{~W} / \mathrm{m}$. Eventually data for $\mathrm{CO}_{2}$ would have to be included in this type of analysis, but even allowing for this it seems clear that the liner parameters assumed for the calculations in Figures 25 and 26 are close to what would have permitted satisfactory operation of the SSC.

(a)

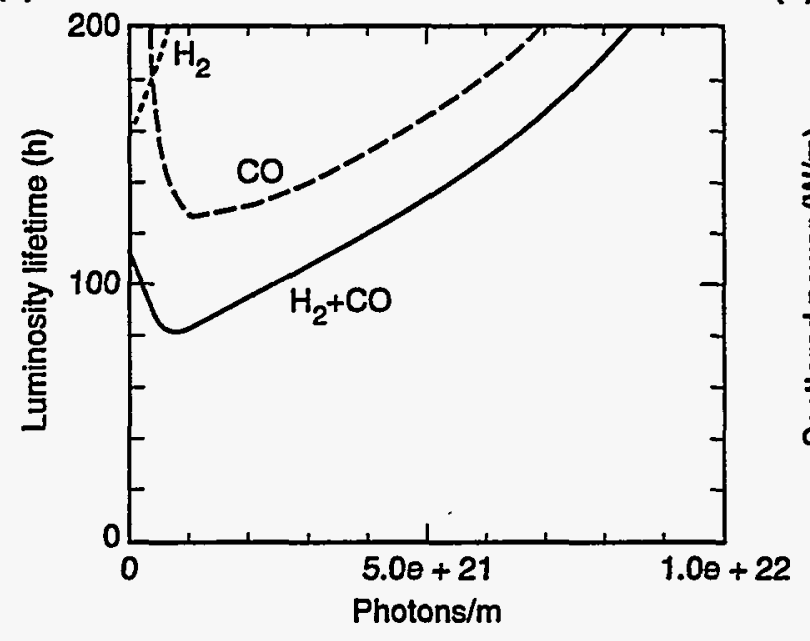

(b)

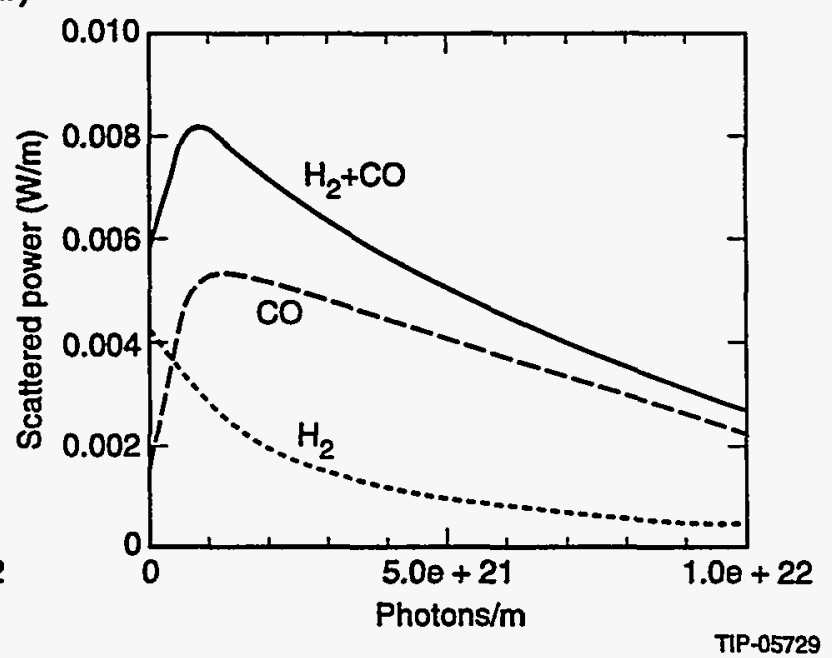

Figure 26. (a) Estimated SSC vacuum-limited luminosity lifetime; (b) beam power scattered by beam gas interactions versus integrated photon flux for a liner with $3002-\mathrm{mm}$-diameter holes $/ \mathrm{m}$. The photon intensity is $10^{16}$ photons $/ \mathrm{m} / \mathrm{s}$.

The fraction of wall area covered by circular holes for the calculations in Figures 25 and 26 is $0.94 \%$. From the results given in Figure 18, Section 1.5.1, and Section 5 the transverse and longitudinal impedances are increased by only $10 \%$ compared to no liner and the same 
beam tube diameter. Consequently the single-bunch instability safety margins are essentially not affected for this case. A further reduction of the hole impedance contribution by roughly a factor of two could be obtained by using rounded-end slots with width equal to the hole diameter and length-to-width ratio equal to three.

\section{New Techniques for Measurement of Gas Density in a Cryosorbing Beam Tube}

It is desirable to have methods of direct gas-density measurement inside a cryosorbing beam tube. If this is done, both the gas density and the mean molecular velocity that appear in the model equations can be independently determined. Two methods that have been under investigation in connection with the work at SSCL since 1991 will be briefly described in this section.

\section{1. $H^{+} / H^{-}$Ion Beam Method}

The $\mathrm{H}^{+} / \mathrm{H}^{-}$ion beam method uses various stripping or charge-exchange reactions of $\mathrm{H}^{-}$ or $\mathrm{H}^{+}$beams on neutral gas molecules $(X)$-for example, $\mathrm{H}^{+}+X \longrightarrow \mathrm{H}^{0}+X^{+}, \mathrm{H}^{+}+X \longrightarrow$ $\mathrm{H}^{-}+\mathrm{X}^{++}$-and is being developed at BINP. Figure 27 shows a diagram of the method for an $\mathrm{H}^{+}$beam. A proton beam $(\sim 20 \mathrm{keV}, \sim 1 \mu \mathrm{A})$ is extracted from an rf ion source, transported to the cryostat, and injected along the axis of the beam tube by a bending magnet. $\mathrm{H}^{0}$ and $\mathrm{H}^{-}$produced by interaction with beam gas molecules are separated from each other and $\mathrm{H}^{+}$ by another bending magnet at the beam tube exit and are detected with secondary electron multipliers (SEMs). Two pairs of small superconducting dipole magnets near the center of the beam tube are used to offset a $\sim 20-\mathrm{cm}$ segment of the $\mathrm{H}^{+}$beam orbit. The SEMs are lined up to observe $\mathrm{H}^{0}$ and $\mathrm{H}^{-}$produced along this offset segment, thus providing a localized measurement of beam gas density. If necessary, background due to synchrotron radiation photons can be discriminated against by gating the detector on between bursts of synchrotron radiation. For the double-charge exchange reaction $\mathrm{H}^{+}+\mathrm{H}_{2} \longrightarrow \mathrm{H}^{-}+\mathrm{H}_{2}{ }^{++}$, which should be insensitive to synchrotron radiation background, we can estimate the signal intensity from

$$
R=\epsilon n \sigma_{1,-1} L I_{\mathrm{H}+},
$$

where $n$ is the $\mathrm{H}_{2}$ gas density $\left(3 \times 10^{8} / \mathrm{cm}^{3}\right), \sigma_{1,-1}$ the cross section for $\mathrm{H}^{+}+\mathrm{H}_{2} \longrightarrow \mathrm{H}^{-}+\mathrm{H}_{2}{ }^{++}$ $\left(\sim 2 \times 10^{-18} \mathrm{~cm}^{2}\right.$ at $\left.10 \mathrm{keV}\right), L$ the path length of observation $(\sim 20 \mathrm{~cm}), I_{\mathrm{H}^{+}}$the proton current $(\sim 1 \mu \mathrm{A})$, and $\epsilon$ the detector efficiency $(\sim 1.0)$. Inserting the numbers in parentheses we get $R=7.5 \times 10^{4}$ counts $/ \mathrm{s}$. This is a high-sensitivity method, and the single-charge exchange channel will be even more sensitive, although more susceptible to background from synchrotron radiation. It may be possible to observe the decay of the neutral gas density following fast extraction of the VEPP2M electron beam, thereby obtaining an estimate of the product of sticking coefficient and mean molecular speed. Since this paper has been in preparation, the first results have been obtained, giving a mean speed of photodesorbed $\mathrm{H}_{2}$ molecules, $\bar{v}=8 \pm 1.5 \times 10^{4} \mathrm{~cm} / \mathrm{s}^{40}$ 


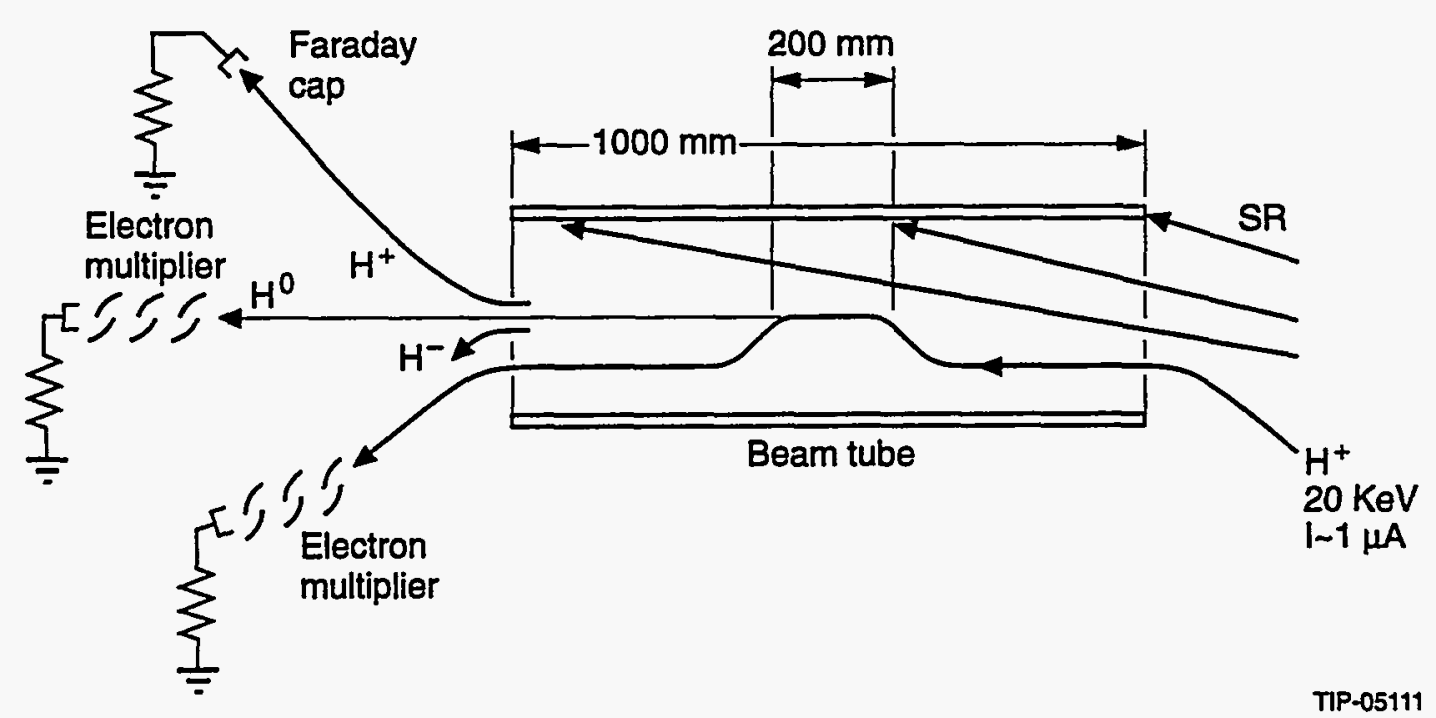

Figure 27. Schematic of the $\mathrm{H}^{+} / \mathrm{H}^{-}$ion beam method of gas density measurement in a cryosorbing beam tube.

\subsection{Positron Method}

A schematic of the proposed BNL positron method of gas density measurement inside a 4.2-K beam tube photodesorption experiment is shown in Figure $28 .{ }^{55}$ Photodesorbing synchrotron radiation photons are incident from the right and are intercepted on the beam tube wall at $\sim 10 \mathrm{mrad}$. For clarity they are omitted from the diagram. Positrons from a ${ }^{22} \mathrm{Na}$ source on the left $\left(e^{+}\right.$end point $=0.540 \mathrm{MeV}$ ) are moderated with a tungsten foil. Moderation efficiency is $\sim 1-5 \times 10^{-4}$. The moderated $e^{+}$leaving the foil are accelerated to the peak of the positronium formation cross section $\sim 30 \mathrm{eV}$ and are separated from high-energy unmoderated $e^{+}$with a pair of $E \times B$ filters. The offset path of the $E \times B$ filters allows for absorption of the unmoderated high-energy $e^{+}$and shields ${ }^{22} \mathrm{Na}$ gamma radiation from the experiment. An electrostatic mirror reflects the positrons from the left, doubling the sensitivity; the second $E \times B$ filter deflects returning $e^{+}$onto a beam dump. A fraction of positrons in the beam tube captures an electron from gas molecules in the tube to form positronium; the pair of $0.511-\mathrm{MeV}$ annihilation gammas is detected in coincidence with $\mathrm{NaI}$ crystal detectors. A magnetic field $B \sim 50-100 \mathrm{G}$, produced by an axial array of Helmholtz coils, is used to guide the $e^{+}$beam. The signal rate $R$ may be estimated from

$$
R=\epsilon^{2}\left(\frac{2 \Omega}{4 \pi}\right) n \sigma L I_{e^{+}}
$$

where $\Omega(\approx \pi \mathrm{sr})$ is the smallest solid angle of the two NaI detectors, $n\left(\approx 3 \times 10^{8} \mathrm{H}_{2} / \mathrm{cm}^{3}\right)$ is the gas density, $\sigma\left(\approx 4 \times 10^{-16} \mathrm{~cm}^{2}\right.$ for $\left.\mathrm{H}_{2}\right)$ is the cross section for formation of positronium, $L(\approx 14.0 \mathrm{~cm})$ is the length of the interaction region viewed by the detectors, $I_{e^{+}}\left(\approx 3 \times 10^{5} / \mathrm{s}\right.$ for a $45-\mathrm{mCi}^{22} \mathrm{Na}$ source) is the number of $e^{+}$per second passing through the interaction region, and $\epsilon(\approx 0.4)$ is an overall detector-efficiency factor including attenuation of the gamma rays by the walls of the cryostat. If we insert the numbers given in parentheses we obtain $\sim 150$ counts $/ \mathrm{h}$, at least an order of magnitude greater than the anticipated random coincidence background. This 
counting rate will be adequate for the usual photodesorption experiments in which desorption coefficients are measured over periods of days.

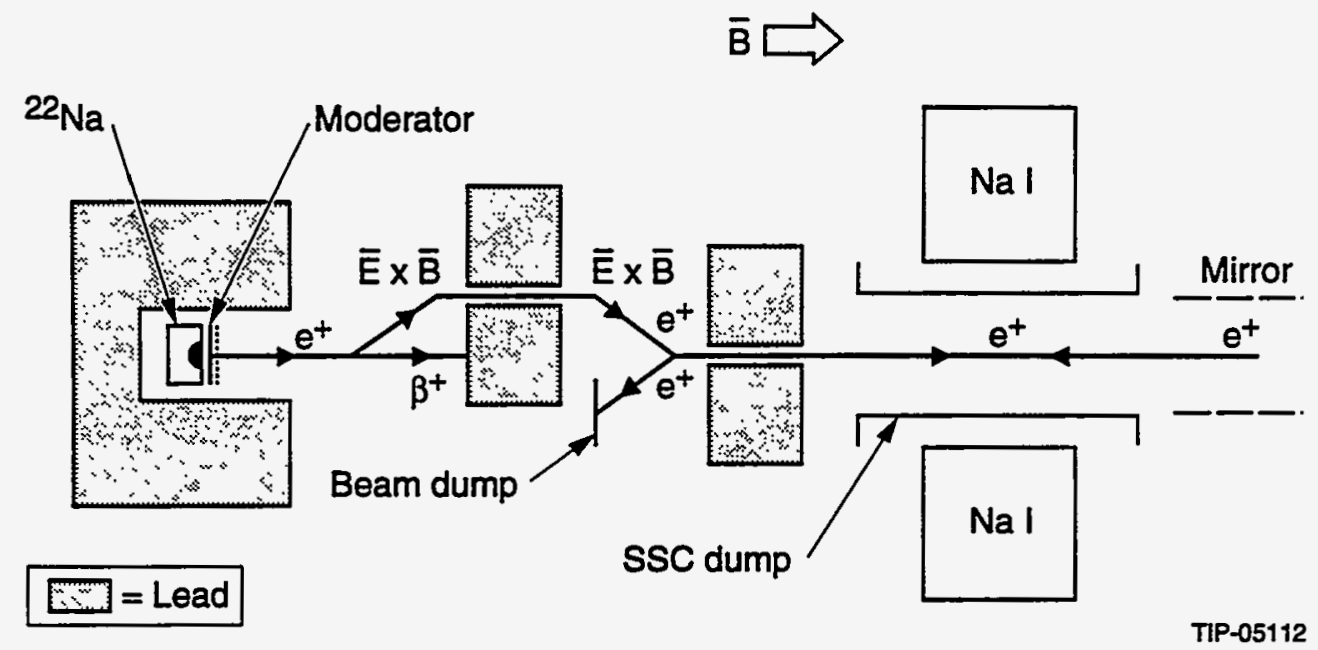

Figure 28. Schematic of the positron method of gas density measurement in a cryosorbing beam tube.

\section{Beam Tube Resistivity}

\subsection{Low Frequency}

A formula for the growth time of the resistive wall instability $\tau_{r w}$, neglecting multi-layer effects, is given by Eq. (10) in Section 1.5.2. Here we will give numerical estimates using the lowfrequency magnetoresistivity measured for copper. In all cases we take the fractional betatron tune $\Delta \nu=0.1$. Figure 29 shows two plots from Reference 56. The first one, Figure 29(a), shows a Kohler plot of the transverse dc magnetoresistivity measured for several samples of electrodeposited $\mathrm{Cu}$. The fractional change in resistivity $\Delta \rho / \rho_{0}$ is plotted versus the product of the magnetic field strength and the field-free residual resistivity ratio $R R=\rho_{0}(273 \mathrm{~K}) / \rho_{0}(\mathrm{~T})$. The measurements were carried out with beam tube samples at $4.2 \mathrm{~K}$ and $77 \mathrm{~K}$, with the transverse magnetic field varying up to $8 \mathrm{~T}$. The symbols are for electrodeposited copper and the solid lines are bounds from a large compilation of results for bulk copper. ${ }^{57}$ The measurements on electrodeposited copper are consistent with bulk copper. The dashed line in Figure 29(a) is an approximate upper bound useful for estimates of $\tau_{r w}$ :

$$
\frac{\Delta \rho(T, B)}{\rho_{0}(T)}=4 \times 10^{-3} B(T) * R R .
$$

The total resistivity at temperature $T$ and magnetic field $B$, then, is

$$
\rho(T, B)=\left[4 \times 10^{-3} B(T)+\frac{1}{R R}\right] * \rho_{0}(273 \mathrm{~K}) .
$$

In this approximate expression all the field dependence is in the first term in brackets, and all the temperature dependence is in the second term. A convenient compilation of the temperature 
dependence of copper resistivity is given in Reference 57. For $R R R=R R(T=4.2 \mathrm{~K})>200$, the resistivity may be taken to be constant $\left(=1.55 \times 10^{-8} / R R R \Omega-\mathrm{m}\right)$ for $T<20 \mathrm{~K}$. One easily sees that at room temperature and for magnetic fields less than $10 \mathrm{~T}$, the magnetoresistivity is at most a few percent correction. At low temperatures, however, the magnetoresistivity term can easily dominate. The second plot from Reference 56, Figure 29(b), shows the product of conductivity and copper thickness $\left(=\sigma_{c t}\right)$ versus magnetic field strength for a $100-\mu \mathrm{m}$-thick copper layer and two values of $R R R=\rho_{0}(273 \mathrm{~K}) / \rho_{0}(4.2 \mathrm{~K})$, corresponding to the best $(R R R=270)$ and worst $(R R R=65)$ cases in the samples tested. At high fields, corresponding to flattop energy in a storage ring, the $R R R$ value makes little difference, whereas for fields less than $1 \mathrm{~T}$, corresponding to injection, the $R R R$ value makes an appreciable difference. Since $\tau_{r w}$ increases linearly with $\gamma$ while the product $\sigma_{c} t$ decreases more slowly than $1 / B$, the growth time at injection is the worst situation. For SSC the design field was $0.67 \mathrm{~T}$ at injection, and a product $\sigma_{c} t>1-2 \times 10^{5} \Omega^{-1}$ led to resistive wall growth times greater than 100 turns and was regarded as acceptable for feedback stabilization. This condition was met for all of the samples tested in Reference 56, assuming a copper thickness $t=100 \mu \mathrm{m}$.

(a)

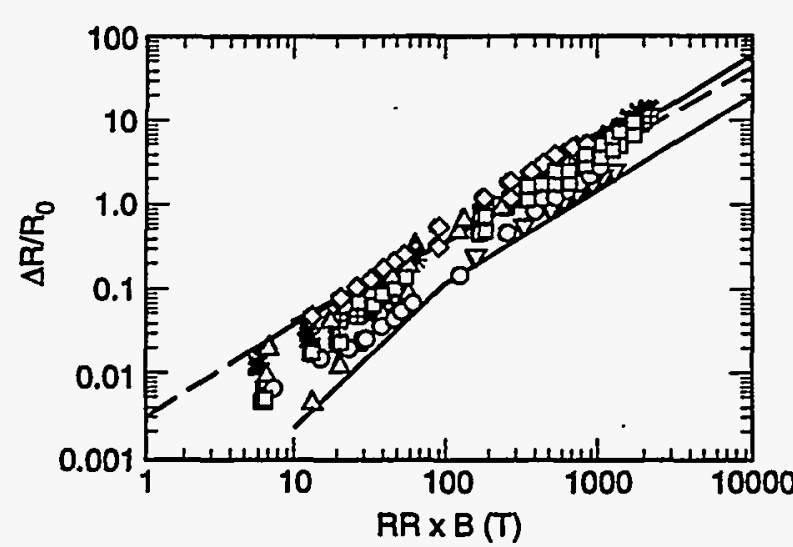

(b)

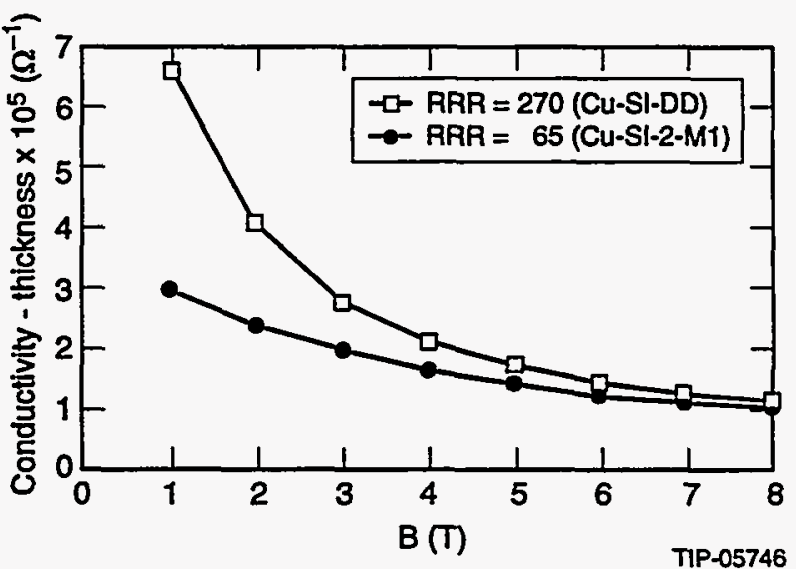

Figure 29. (a) Magnetoresistivity of electrodeposited $\mathrm{Cu}$; (b) product of conductivity times thickness versus magnetic field strength for a $100-\mu \mathrm{m}$-thick layer of electrodeposited $\mathrm{Cu}^{56}$

In Table 6 we have used the parameters of the SSC, LHC, and ELN together with Eq. (69) for the magnetoresistivity to estimate the growth rates for the resistive wall instability at injection energy. In all cases we have assumed a copper thickness of $100 \mu \mathrm{m}$. The SSC and LHC both have rather long growth times of hundreds of turns and present no difficulty for feedback stabilization. The ELN growth time is only a few turns and would require some adjustment of parameters. We note that if the beam tube were stainless steel without the copper layer, then $\sigma_{c} t \approx 2.0 \times 10^{3} \Omega^{-1}$ and the resistive wall growth times for the SSC and LHC would be reduced to unacceptable values of the order of a few turns. 
Table 6. Multi-bunch resistive wall instability growth rates.

\begin{tabular}{|l|c|c|c|}
\hline Parameter & SSC & LHC & ELN \\
\hline$M$ & 17424 & 2835 & 81000 \\
\hline$N_{b}$ & $0.75 \times 10^{10}$ & $10.0 \times 10^{10}$ & $3.5 \times 10^{10}$ \\
\hline$\beta_{\text {avg }}(\mathrm{m})$ & 112 & 62 & 492 \\
\hline$\gamma_{\text {inj }}$ & $2.1 \times 10^{3}$ & $0.48 \times 10^{3}$ & $5.3 \times 10^{3}$ \\
\hline$b(\mathrm{~mm})$ & 16 & 20 & 15 \\
\hline$\tau_{0}(\mathrm{~ms})$ & 0.294 & 0.089 & 1.185 \\
\hline$\tau_{\mathrm{rw}}(\mathrm{ms})$ & 77 & 29 & 1.83 \\
\hline$\tau_{\mathrm{rw}} / \tau_{0}$ & 262 & 325 & $\sim 3$ \\
\hline
\end{tabular}

\subsection{High Frequency}

A convenient expression for the heating of the beam tube wall by the beam image current is given by Eq. (12) in Section 1.5.2. The main concern is that the heating not be too large to be absorbed at cryogenic temperatures. It turns out that once a high-conductivity layer has been specified for stabilization of the resistive wall instability, wall heating by the beam image current falls into an acceptable range. The details of the resistivity are different, however, owing to the high frequencies $\left(f \approx \frac{1}{2 \pi} \frac{c}{\sigma_{z}} \approx 1 \mathrm{GHz}\right)$ characteristic of the bunched current. At $f=1 \mathrm{GHz}$ and for $R R R=100$, the classical skin depth in copper is $\delta=0.2 \mu \mathrm{m}$, so the image current flows in a thin-surface layer much less than the thickness of the copper. The product of electron mean free path and resistivity is a material property independent of temperature, purity, and frequency; for copper it has the value $6.6 \times 10^{-16} \Omega-\mathrm{m}^{2}$. At low temperature the electron mean free path, 
therefore, increases by the $R R$ ratio and can exceed the skin depth (e.g., for $R R R=100$, the mean free path is $4.26 \mu \mathrm{m}$ for $T<20 \mathrm{~K}$ ). In this case the classical relation $R_{s}=\rho / \delta$ with $\delta=\left(\rho / \pi \mu_{0} f\right)^{1 / 2}$ is no longer valid, and one is in the anomalous skin-depth regime. A useful description of this situation is given in Reference 58, which also contains measurements of the surface resistivity of copper from $1 \mathrm{~K}$ to room temperature in the $\mathrm{GHz}$ frequency range and with magnetic fields up to $6 \mathrm{~T}$. According to Reference 58 a dimensionless parameter characterizing the division between classical and anomalous regimes is $\alpha=(3 / 4) \mu_{0} \omega(\rho \lambda)^{2} / \rho^{3}$, where $\lambda$ is the electron mean free path. The classical regime pertains when $\alpha<0.02$, whereas for $f=1 \mathrm{GHz}$ and $R R R=100, \alpha=693$, clearly in the anomalous regime. In this case the surface resistance is represented by:

$$
R_{s}=R_{\infty}\left(1+\frac{1.157}{\alpha^{0.276}}\right)
$$

where $R_{\infty}=\left[\frac{\sqrt{3} \pi}{4} \mu_{0}^{2}(\rho \lambda) f^{2}\right]^{1 / 3}$. For copper at $f=1 \mathrm{GHz}, R_{\infty}=1.13 \times 10^{-3} \Omega$. The bulk copper used in Reference 58 was measured to have $R R R=180$, whereas the experimental data at low temperature are fit with Eq. (70), with $R R R=24$, presumably because of surface conditions different from those in the bulk material. The surface resistance was observed to be constant for $T<20 \mathrm{~K}$ and for $f=1 \mathrm{GHz}, R_{s}=1.8 \times 10^{-3} \Omega$. The magnetoresistivity was also measured in the $\mathrm{GHz}$ range and was found to be less than $3 \%$ up to $6 \mathrm{~T}$. This is much less than the magnetic dependence at low frequency given by Eq. (69), and we will not concern ourselves with it any further here.

Since surface conditions appear to have an important effect, a collaboration with LANL was begun at the SSC to measure the high-frequency surface resistance of actual SSC beam tube materials. Since $1 \mathrm{GHz}$ is below the beam tube cutoff frequency, cavity modes cannot be excited as in Reference 58. Instead, the TEM mode was excited in resonant lengths of beam tube with a superconducting niobium center conductor. ${ }^{59}$ Several samples of electrodeposited $\mathrm{Cu}$ beam tube were measured at $f=1 \mathrm{GHz}$, and a representative value obtained was $R_{s}=2 \times 10^{-3} \Omega$, remarkably close to the result in Reference 58 for bulk high-purity electronic copper. We have used $R_{s}=2 \times 10^{-3} \Omega$ to evaluate the image current heating according to Eq. (12); the results are shown in Table 7 for SSC, LHC, and ELN assuming in all cases a surface temperature of $20 \mathrm{~K}$ or less. For the SSC and ELN the image current heating power is negligible compared to the synchrotron radiation power given in Table 1. For the LHC it is a fairly large fraction (73\%) of the magnitude of synchrotron power, and although still manageable it needs consideration in the overall design of the cryogenic refrigeration. 
Table 7. Estimates of beam image parasitic heating.

\begin{tabular}{|l|c|c|c|}
\hline Parameter & SSC & LHC & ELN \\
\hline$M$ & 17424 & 2835 & 81000 \\
\hline$N_{b}$ & $0.75 \times 10^{10}$ & $10.0 \times 10^{10}$ & $3.5 \times 10^{10}$ \\
\hline$b(\mathrm{~mm})$ & 16 & 20 & 15 \\
\hline$\sigma_{z}(\mathrm{~cm})$ & 7 & 7.5 & 7.8 \\
\hline $\mathrm{P}(\mathrm{W}) /$ ring & 125 & 2700 & 12180 \\
\hline
\end{tabular}

\section{Summary}

Achieving a satisfactory beam tube vacuum in the next-generation superconducting proton collider presents a significant challenge for accelerator science and technology. The beam tube vacuum system will need to be designed to pump the gas photodesorbed by synchrotron radiation in order to avoid degradation of the luminosity lifetime and possibly the quenching of superconducting magnets due to beam scattering. For the first time the magnitude of synchrotron radiation power will need to be taken into consideration in the design of the cryogenic refrigeration system. The physical space allowed for pumping and heat removal is limited by the high cost of superconducting magnet aperture and the need for physical aperture for the circulating beams. Experiments have shown that cryosorption on a simple beam tube coinciding with the superconducting magnet bore tube won't work because of the very high photodesorption coefficient of physisorbed gas. The photodesorbed gas must be pumped out of the view of the direct and diffusely scattered radiation. Fortunately, a viable solution exists in the form of a perforated liner or beam screen coaxial with the magnet bore tube. The liner can be operated above the magnet cryostat temperature, thereby reducing the cryogenic cost of absorbing the synchrotron radiation power. It appears that the increase in impedance by the liner perforations will not greatly reduce the safety margin for single-bunch instabilities. Quite a lot has been learned in the relatively short time that the SSC and LHC have been under consideration. Experimental techniques are in hand for obtaining the information necessary for a quantitative design and prediction of performance of the beam tube vacuum system. In the future we can look forward to improvements in most of the areas discussed in this paper regarding photodesorption of gases 
inside cold beam tubes exposed to synchrotron radiation: direct measurement of gas density, determination of the molecular speed, measurement of the photodesorption coefficient of $\mathrm{CO}_{2}$, and determination of sticking coefficients. In this paper we have dealt with understanding the performance of a liner and defining the situations that require it. There are also a number of interesting design, fabrication, and operational issues that have not been discussed and remain to be settled in the building of a real system.

\section{Acknowledgments}

I would like to thank Richard Briggs for attracting my attention to the beam tube vacuum problems in superconducting proton colliders. In addition I have benefited from many informative conversations with Vadim Anashin, Richard Briggs, Roger Calder, Oswald Grobner, Alfred Maschke, Alastair Mathewson, and Myron Strongin during the two-and-a-half years I worked on these problems at the ill-fated SSCL.

\section{References}

1. SSC-Central Design Group, "Conceptual Design of the Superconducting Super Collider (SSC)," SSC-R-2020 (1986).

2. LHC Study Group, "Design Study of the Large Hadron Collider (LHC)," CERN 91-03 (1991).

3. W. Barletta and H. Leutz, eds., Supercolliders and Superdetectors, World Scientific Publishing Co., Singapore (1993).

4. O. Grobner, A. Mathewson, H. Stori, P. Strubin, and R. Souchet, Vacuum, 33, 397 (1983).

5. R. Carcagno, W. Schieller, H.-J. Shih, X. Xu, and A. Yucel, Proceedings of Supercollider IV, New Orleans (1992).

6. W. Chou, Appendix 7 in H. Edwards, SSCL-N-771 (1991).

7. J. D. Jackson, "Surface Impedance of a Two-Layer Beam Tube and Resistive-Wall Contribution to Machine Impedances," SSC-N-110 (Jan. 1986).

8. R.J. Briggs, "Low Frequency Transverse Resistive Instability in the Collider," SSCL-512 (June 1992).

9. Reference 1, p. 177.

10. W. Turner, "Dynamic Vacuum in the Beam Tube of the SSCL Collider-Cold Beam Tube and Liner Options," SSCL-PP-404 (May 1993); Proc. of 1993 IEEE Part. Acc. Conf., Washington, D.C., p. 3833 (1993).

11. W: Turner, "Model of a $80-\mathrm{K}$ Liner Vacuum System for the 4.2-K Cold Bore of the SSCL 20-TeV Proton Collider," SSCL-SR-1224/3 (1993), submitted for publication.

12. S. Brunauer, P. Emmett, and E. Teller, J. Chem. Soc., 60, 309 (1938).

13. C. Benvenuti, R. Calder, and G. Passardi, J. Vac. Sci. Technol., 13, 1172 (1976).

14. M. G. Rao, P. Kneisel, and J. Susta, "Cryosorption Pumping of $\mathrm{H}_{2}$ and He with Metals and Metal Oxides at $4.3 \mathrm{~K}$," Proc. of the 15th International Cryogenics Engineering Conference, Genova, Italy, 6-10 June 1994. CEBAF Tech. Note 94-036. 
15. E. Wallen, "Adsorption Isotherms of $\mathrm{H}_{2}$ on $\mathrm{Cu}$ Plated Stainless Steel," CERN Vacuum Tech. Note 93-17, Dec. 1993.

16. D. Bintinger, P. Limon, H. Jostlein, and D. Trbjovic, "Status of SSC Photodesorption Experiment," SSC-102 (1986).

17. D. Bintinger, P. Limon, and R. Rosenberg, J. Vac. Sci. Technol., A7, 59 (1989).

18. A. G. Mathewson, M. Andritschky, O. Grobner, F. Schumann, R. Souchet, and P. Strubin, "Gas Desorption and Surface Conditioning of a Synchrotron Radiation Source," Vacuum Design of Advanced and Compact Synchrotron Light Sources, AIP Conference Proceedings No. 171, New York (1988).

19. C. L. Foerster, H. Halama, and C. Lanni, J. Vac. Sci. Technol., A8, 2856 (1990).

20. C. L. Foerster, C. Lanni, I. L. Maslennikov, and W. C. Turner, "Photon Desorption Measurements of Copper and Copper Plated Beam Tubes for the SSCL $20 \mathrm{TeV}$ Proton Collider," J. Vac. Sci. Technol., A12, 1673 (1994).

21. E. Garwin, "3 BeV Colliding Beam Vacuum System," SLAC memorandum dated 14 Aug. 1963.

22. O. Grobner, A. G. Mathewson, and P. C. Marin, "Gas Desorption from an OFHC Copper Vacuum Chamber by Synchrotron Radiation Photons," J. Vac. Sci. Technol., A12, 846 (1994).

23. J. Gomez-Goni, O. Grobner, A. G. Mathewson, and A. Poncet, "Continued Exposure of a Stainless Steel Chamber to Photons," CERN, Vacuum Technical Note 92-03, Feb. 1992.

24. F. Biggs and R. Lighthill, "Analytical Approximations for X-Ray Cross Sections III," Sandia National Laboratory report SAND87-0070 (1988).

25. C. J. Powell, Surface Science, 44, 29 (1974).

26. M. D. Maleev, "Gas Absorption and Outgassing of Metals," Vacuum, 23, 43 (1972).

27. M. Andritschky, O. Grobner, A. G. Mathewson, F. Schumann, P. Strubin, and R. Souchet, "Synchrotron Radiation Induced Neutral Gas Desorption from Samples of Vacuum Chambers," Vacuum, 38, 933 (1988).

28. G. Dugan, "Hydrogen Density Distribution in SSC Beam Pipe Materials and Connection to Photodesorption Coefficients in a Diffusion Model," SSCL-N-863 (1994).

29. I. Maslennikov, W. Turner, V. Anashin, O. Malyshev, et al., "Photodesorption Experiments on SSC Collider Beam Tube Configurations," SSCL-PP-378; Proc. of 1993 IEEE Part. Acc. Conf., Washington, D.C., p. 3876 (1993).

30. R. S. Calder, Vacuum, 24, 437 (1974).

31. N. Hilleret and R. Calder, Proc. of 7th Intern. Vac. Congress and 3rd Intern. Conf. Solid Surfaces, p. 227, (Vienna, 1977).

32. C. L. Foerster, H. Halama, G. Korn, M. Calderon and W. Barletta, "Desorption Measurements of Copper and Copper Alloys for PEP-II," Proc. of the 12th International Vacuum Congress (1992). 
33. B. He, M. Stawovy, G. Lu, S. Desu, W. Reynolds, and A. Aning, "Influence of Heat Treatment on Photodesorption of Copper Plated SSCL Beam Tube," SSCL-N-849 (1994).

34. "Site-Specific Conceptual Design of the Superconducting Super Collider," SSCL-SR-1056, pp. 241-242 (July 1990).

35. A. Maschke, "Hydrogen Desorption and the Search for the Higgs," Proc. of the Fifth International Workshop on Desorption Induced by Electronic Transitions, Taos, N.M., Apr. 1-4, 1992; SSCL-PP-86 (March 1992); and A. Maschke, Appendix 1 in H. Edwards, SSCL-N-771 (1991).

36. V. Anashin, A. Evstigneev, O. Malyshev, V. Osipov, I. Maslennikov, and W.Turner, "Summary of Recent Photodesorption Experiments at VEPP2M," SSCL-N-825 (June 1993).

37. V.V. Anashin, O.B. Malyshev, V.N. Osipov, I.L. Maslennikov, and W.C. Turner, "Investigation of Synchrotron Radiation Induced Photodesorption in Cryosorbing Quasi-Closed Geometry," SSCL-PP-517 (October 1993); J. Vac. Sci. Tech., A12, 2917 (1994).

38. V.V. Anashin et al., "Cold Beam Tube Photodesorption Experiments for the SSCL 20-TeV Proton Collider," SSCL-PP-533 (November 1993); J. Vac. Sci. Tech., A12, 1663 (1994).

39. V.V. Anashin, O.B. Malyshev, V.N. Osipov, I.L. Maslennikov, and W.C. Turner, "Experimental Investigation of Dynamic Pressure in a Cryosorbing Beam Tube Exposed to Synchrotron Radiation," SSCL-PP-562 (June 1994); to appear in Proc. of 1994 European Particle Accelerator Conference, London (1994).

40. N. Alinovsky, V. Anashin, P. Beschasny, G. Derevyankin, V. Dudnikov, A. Evstigneev, O. Malyshev, V. Osipov, I. Maslennikov, and W. Turner, "A Hydrogen Ion Beam Method of Molecular Density Measurement Inside a 4.2-K Beam Tube," SSCL-PP-563 (June 1994); to appear in Proc. of 1994 European Particle Accelerator Conference, London (1994).

41. M. Ruckman, M. Strongin, W. Lanford, and W. Turner, "Nuclear Reaction Analysis of Hydrogen in Candidate SSC Beam Pipe Materials," BNL-60228 (1994); submitted for publication.

42. F. Jacobsen, M. Ruckman, and M. Strongin, report in preparation.

43. S. Anderson, L. Wilzen, M. Persson, and J. Harris, Phys. Rev., B40, 8146 (1989).

44. S.S. Kurennoy, Part. Acc., 39 (1992), 1.

45. R. L. Gluckstern, Phys. Rev. A, 46 (1992), 1106, and Phys. Rev. A, 46 (1992), 1110.

46. E. D. Ruiz, L. S. Walling, Y. Goren, and N.J. Spayd, Proc. of IEEE 1993 PAC, 3405.

47. W. Chou and T. C. Barts, Proc. of IEEE 1993 PAC, 3444.

48. K. Leung, "Eddy Current and Quench Stress of SSCL 4-K Liner and Bore Tube," Advances in Cryogenic Engineering, 39, 771 (1994), Plenum Press, N.Y.

49. S. S. Kurennoy, "Pumping Slots: Coupling Impedance Calculations and Estimates," SSCL$636(1993)$.

50. S. S. Kurennoy, "Trapped Modes in Waveguides with Small Discontinuities," to appear in Proc. of 1994 European Particle Accelerator Conference, London (1994). 
51. L. Walling, T. Barts, E. Ruiz, N. Spayd, and W. Turner, "Above Cutoff Impedance Measurements of Pumping Holes for the Collider Liner," SSCL-670 (Apr. 1994).

52. H. Ishimaru, K. Narushima, K. Kanazawa, Y. Suetsugu, D. Bintinger, H. Jostlein, and D. Trobojevic, J. Vac. Sci. Technol., A6, 1293 (1988).

53. W. Chou, "Feasibility Study of Aluminum Beam Tube for the Collider-An Option for No-Coating and No-Liner," SSCL-649 (1994).

54. M. G. Rao, P. Kneisel, and J. Susta, "Cryosorption Pumping of $\mathrm{H}_{2}$ and He with Metals and Metal Oxides at 4.3 K," CEBAF Tech. Note 94-036, Proc. of the 15th International Cryogenics Engineering Conference, Genova, Italy, 6-10 June 1994.

55. F. Jacobsen, A. Wiess, M. Strongin, M. Ruckman, and W. Turner, "The Development of a Positron Ionization Gage," SSCL-N-846 (1994).

56. V. T. Kovachev, M. J. Neal, J. Zbasnik, M. Tule, P. G. Cline, F. Y. Clark, Q. S. Shu, and J. M. Seuntjens, "Magnetoresistance of SSC Beam Tube Samples," Advances in Cryogenic Engineering, 39, 479 (1994), Plenum Press, N.Y.

57. N. J. Simon, E. S. Drexler, and R. P. Reed, Properties of Copper and Copper Alloys at Cryogenic Temperatures, NIST Monograph 177, U.S. Dept. of Commerce (1992).

58. J. T. Rogers, S. DePanfilis, A. C. Melissinos, B. E. Moskowitz, et al., Appl. Phys. Lett., 52, 2266 (1988).

59. E. R. Gray, "SSC Beam Tube Resistance Measurements," LANL Tech. Note AT-1:93-344 (Dec. 1993).

\section{Appendix-Three-component Formulation of the Model Vacuum Equations}

In this appendix we write down the three-component formulation of the model vacuum equations. The density equations are summed to give the single-component model represented by Eqs. (13) and (14) in the main text of the paper, with density-averaged diffusion coefficient, liner conductance, wall sticking coefficient, and pumping speed. The equivalence of the infinitelength beam tube solutions is discussed. If we allow subscripts 1 to designate molecules having their origin in the photodesorption of tightly bound hydrogen, 2 to designate photodesorption of physisorbed molecules, and 3 to designate thermal desorption of physisorbed hydrogen, then the diffusion equations for each of these components is

$$
\begin{aligned}
& V \frac{\partial n_{1}}{\partial t}=\eta_{1} \dot{\Gamma}-\sigma_{w, 1} S_{w, 1} n_{1}-C_{1} n_{1}+A_{c} D_{1} \frac{\partial^{2} n_{1}}{\partial z^{2}} \\
& V \frac{\partial n_{2}}{\partial t}=\eta^{\prime} \dot{\Gamma}-\sigma_{w, 2} S_{w, 2} n_{2}-C_{2} n_{2}+A_{c} D_{2} \frac{\partial^{2} n_{2}}{\partial z^{2}} \\
& V \frac{\partial n_{3}}{\partial t}=\frac{A_{w} s}{\tau_{w}^{t}}-\sigma_{w, 3} S_{w, 3} n_{3}-C_{3} n_{3}+A_{c} D_{3} \frac{\partial^{2} n_{3}}{\partial z^{2}}
\end{aligned}
$$


and the equation for the surface density of physisorbed $\mathrm{H}_{2}$ is

$$
A_{w} \frac{\partial s}{\partial t}=\eta_{2} \dot{\Gamma}+\sum_{j} \sigma_{w, j} S_{w, j} n_{j}-\frac{A_{w} s}{\tau_{w}^{t}}-\eta^{\prime} \dot{\Gamma}
$$

If we sum Eqs. (A1) we get

$$
V \frac{\partial n}{\partial t}=\eta_{1} \dot{\Gamma}+\eta^{\prime} \dot{\Gamma}+\frac{A_{w s}}{\tau_{w}^{t}}-\sigma_{w} S_{w} * n-C * n+A_{c} D \frac{\partial^{2} n}{\partial z^{2}},
$$

which is simply Eq. (13) with $n, \sigma_{w} S_{w}, C$, and $D$ defined by the following density-weighted averages:

$$
\begin{aligned}
n & =\sum_{j} n_{j} \\
\sigma_{w} S_{w} & =\frac{\sum_{j} \sigma_{w, j} S_{w, j} n_{j}}{n} \\
C & =\frac{\sum_{j} C_{j} n_{j}}{n} \\
D & =\frac{\sum_{j} D_{j} n_{j}}{n} .
\end{aligned}
$$

Using these same definitions we also recover Eq. (14) for surface density.

If Eqs. (A1) are solved for an infinitely-long tube with $V \frac{\partial n}{\partial t} \approx 0, C=0$, axial diffusion neglected, and $A \frac{\partial s}{\partial t} \neq 0$, we get

$$
\begin{aligned}
n & =n_{1}+n_{2}+n_{3} \\
& =\frac{\eta_{1} \dot{\Gamma}}{\sigma_{w, 1} S_{w, 1}}+\frac{\eta^{\prime} \dot{\Gamma}}{\sigma_{w, 2} S_{w, 2}}+\frac{1}{\sigma_{w, 3} S_{w, 3}} \frac{A_{w} s}{\tau_{w}^{t}} .
\end{aligned}
$$

On the other hand, solving Eq. (A3) in the same approximation as was done in the main body of the paper (Eq. (26)) gives

$$
n=\frac{\eta_{1} \dot{\Gamma}}{\sigma_{w} S_{w}}+\frac{\eta^{\prime} \dot{\Gamma}}{\sigma_{w} S_{w}}+\frac{1}{\sigma_{w} S_{w}} \frac{A_{w} s}{\tau_{w}^{t}}
$$

Equations (A5) and (A6) are equivalent by virtue of the definitions given in (A4). Equations (A5) and (A6) are not equal term-by-term, however, so properly speaking the gas density of each of the three components is given correctly only by the components of Eq. (A5). 